\title{
Mitigating Security Problems in Virtualized Networks Through Resource Management
}

by

\section{Danish Sattar}

A dissertation submitted to the Faculty of Graduate and Postdoctoral Affairs in partial fulfillment of the requirements for the degree of

\author{
Doctor of Philosophy \\ in
}

Electrical and Computer Engineering

Ottawa-Carleton Institute for Electrical and Computer Engineering

Department of Systems and Computer Engineering

Carleton University

Ottawa, Ontario

September, 2020

(C) 2020

Danish Sattar 


\section{Abstract}

Network Virtualization is the key to the current and future success of the Internet, and it has proven to be one of the core technologies in the evolution of the Internet. The virtualization of network resources offers many benefits. Resources are more efficiently utilized, and they can be deployed flexibly and elastically. It can lower the cost of ownership by moving from dedicated hardware to virtual resources. Another important benefit is the ease of management through standard abstractions. However, these benefits come at the cost of security. Network virtualization has increased the threat surface due to the virtualization of resources. Such as risk of isolation failure, privacy, and confidentiality of hosted services, side-channel attacks, and amplified impact of Denial-of-Service attacks.

In this dissertation, we take a look at some of the security issues in the virtualized networks. We aim to utilize resource management to mitigate some of the security problems in the virtualized networks. In particular, we use Software-Defined Network-

ing $(\mathrm{SDN})$ and $5 \mathrm{G}$ mobile networks as a focus of our study to investigate and mitigate security issues. We identified that Distributed Denial-of-Service (DDoS) attacks pose a significant risk in SDN and emerging $5 \mathrm{G}$ mobile networks because both networks are virtualized, and the impact of DDoS is amplified. In a traditional network, there are usually very few stakeholders that are impacted by the DDoS. Whereas, in virtualized networks, many tenants share the same resources; therefore, many stakeholders can 
be impacted (e.g., from infrastructure provider to network operator to the tenants). We use resource management to deal with DDoS attacks. In SDN, load-balancing is used to deal with DDoS attacks. Whereas, in 5G mobile networks, we proposed an optimization-based resource allocation solution to mitigate DDoS attacks. We also analyze the defense against malicious co-residency using our solution in 5G mobile networks. Last but not least, we proposed a framework for resource allocation in the 5G mobile networks. 
Dedicated to my Beloved Parents 


\section{Acknowledgments}

First, I would like to express my sincere gratitude to my supervisor, Prof. Ashraf Matrawy, for his continuous support, patience, motivation, and immense knowledge. His guidance helped me in all the time of research and writing of this thesis. I could not have imagined having a better advisor and mentor for my Ph.D. study.

Besides my advisor, I would like to thank the rest of my thesis committee: Prof. Burak Kantarci, Prof. AbdelRahman Abdou, Prof. Mohammad Mannan, and Prof. Changcheng Huang for their encouragement, insightful comments, and challenging questions.

I thank my fellow labmates for the stimulating discussions and all the fun we have had in the last five years.

Last but not least, I would like to express my indebtedness to my parents, my brothers and sisters, and my best friends for supporting me spiritually throughout the writing of this thesis and my life in general. 


\section{Table of Contents}

Abstract $\quad$ ii

Acknowledgments $\quad$ v

Table of Contents vi vi

List of Tables $\quad$ X

List of Figures $\quad$ xi

List of Abbreviations $\quad$ xiv

List of Symbols $\quad$ xviii

1 Introduction 1

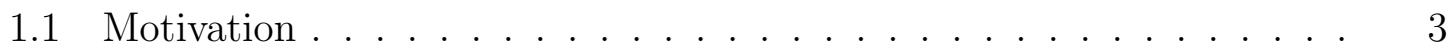

1.2 Scope ................................. 4

1.3 Research Question .................... 4

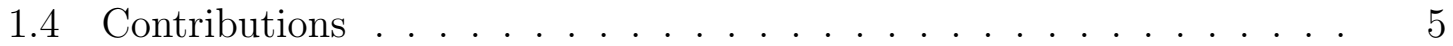

1.5 List of Publications . . . . . . . . . . . . . . . . . . . . 7

1.5.1 Publications included in the thesis . . . . . . . . . . 7

1.5.2 Publications not included in the thesis . . . . . . . . 8 
2 Background $\quad 9$

2.1 Evolution of Networks . . . . . . . . . . . . . . . . . . . . 9

2.1.1 Computer Networks . . . . . . . . . . . . . . . . 9

2.1.2 Mobile Networks . . . . . . . . . . . . . . . . . . . 11

2.2 LTE Network . . . . . . . . . . . . . . . . . . . . . . . 13

$2.35 \mathrm{G}$ Network . . . . . . . . . . . . . . . . . . . . 14

2.3.1 5G Network Slicing . . . . . . . . . . . . . . . . . . 17

3 Research Problem and Methodology 20

3.1 Research Problem . . . . . . . . . . . . . . . . . 20

3.2 Research Methodology . . . . . . . . . . . . . . . . . 22

3.2.1 Primary Data and Tools . . . . . . . . . . . . . . . 22

3.2.2 Research Process . . . . . . . . . . . . . . . . . 22

\section{Adaptive Bubble Burst (ABB): Mitigating DDoS Attacks in} $\begin{array}{ll}\text { Software-Defined Networks } & 24\end{array}$

4.1 Security challenges in Software-Defined Networks . . . . . . . . 25

4.2 Related Work . . . . . . . . . . . . . . . . . . . . 27

4.3 Threat Model . . . . . . . . . . . . . . . . . . . . 29

4.4 Adaptive Bubble Burst (ABB) . . . . . . . . . . . . . . . . . . . 29

4.4.1 ABB Overview .................... 30

4.4.2 Traffic Balancing ... . . . . . . . . . . . . . 31

4.4 .3 Traffic Drop . . . . . . . . . . . . . . . . 33

4.4.4 Server Anonymity . . . . . . . . . . . . . . . . . . 34

4.5 Experimental Setup and Implementation . . . . . . . . . . . . . 35

4.6 Results . . . . . . . . . . . . . . . . . . . . 37

4.6.1 Scenario 1 . . . . . . . . . . . . . . . . 37 
$4.6 .2 \quad$ Scenario $2 \ldots \ldots \ldots \ldots \ldots$

$4.6 .3 \quad$ Scenario $3 \ldots \ldots$. . . . . . . . . . . . . . . . . . . . . . . 39

4.7 Summary . . . . . . . . . . . . . . . . . . . . . 40

5 Optimal Slice Allocation in 5G Core Networks 42

5.1 MILP Formulation . . . . . . . . . . . . . . . . . 45

5.2 Results and Discussion . . . . . . . . . . . . . . . . 49

5.2 .1 Intra-slice isolation . . . . . . . . . . . . . . . . . 51

5.2 .2 End-to-end delay . . . . . . . . . . . . . . . . . . . 54

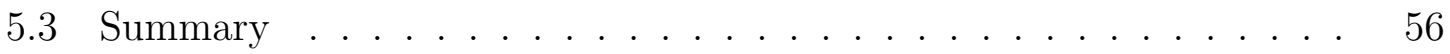

6 Towards Secure Slicing: Using Slice Isolation to Mitigate DDoS Attacks on 5G Core Network Slices $\quad 57$

6.1 Related Work . . . . . . . . . . . . . . . . . . . . . . . . 59

6.2 Threat Model . . . . . . . . . . . . . . . . . . . . . . . . . 61

6.2 .1 Assumptions . . . . . . . . . . . . . . . . . . 61

6.2 .2 Adversaries . . . . . . . . . . . . . . . 62

6.2.3 Attacks on a slice in the multi-tenant networking environment 62

6.3 Proposed Solution . . . . . . . . . . . . . . . . . . . . . . . 64

6.4 Optimization Model Formulation _ . . . . . . . . . . . . 65

6.4.1 Network and Request Model . . . . . . . . . . . . . . . . 65

6.4 .2 Objective function $\ldots \ldots \ldots \ldots \ldots$

6.4.3 General Constraints . . . . . . . . . . . . . . . . . 68

6.4.4 Security-related Constraints . . . . . . . . . . . . . 70

6.5 DDoS Mitigation: Evaluation and Results . . . . . . . . . . 72

6.5.1 Experiment Setup . . . . . . . . . . . . . . . . . 72

6.5.2 Combining simulations with the use of testbed . . . . . . 73 
6.5.3 Test Application . . . . . . . . . . . . . . . . . . 74

6.5.4 Evaluation Methodology . . . . . . . . . . . . . . 75

6.5.5 Results and Discussion . . . . . . . . . . . . . 77

6.6 Performance Evaluation of the Optimization Model . . . . . . . . . 83

6.7 Current Limitations . . . . . . . . . . . . . . . . . . . . . . . . . . . 87

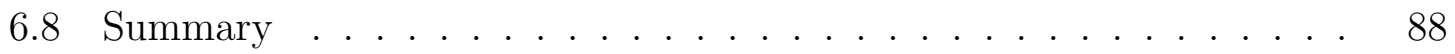

7 Proactive Allocation as Defense for Malicious Co-residency in Sliced 5G Core Networks $\quad 89$

7.1 Related Work . . . . . . . . . . . . . . . . . . 90

7.2 Threat Model . . . . . . . . . . . . . . . . . . . . . . . . . . . 92

7.3 Simulation Setup . . . . . . . . . . . . . . . . . . . 92

7.4 Results and Discussion . . . . . . . . . . . . . . . . 94

7.5 Defense . . . . . . . . . . . . . . . . . . . 95

7.6 Summary . . . . . . . . . . . . . . . . . 96

8 Dynamic Slice Allocation Framework $\quad 97$

8.1 DSAF: Dynamic Slice Allocation Framework . . . . . . . . . . 98

$8.1 .1 \quad$ Experimental Setup . . . . . . . . . . . . . . . . . . 102

8.1.2 Results and Discussion . . . . . . . . . . . . . . . . . . 104

8.2 Qualitative Comparison . . . . . . . . . . . . . . 108

8.3 Summary . . . . . . . . . . . . . . . . . . . 110

9 Conclusion 111

9.1 Current Limitations . . . . . . . . . . . . . . . . . . . . . . . . . . 113

$\begin{array}{ll}\text { List of References } & 115\end{array}$ 


\section{List of Tables}

2.1 Evolution of Mobile Networks . . . . . . . . . . . . . . . . . 13

4.1 ABB experiment parameters . . . . . . . . . . . . . 36

5.1 Variable description of intra-slice isolation optimization model . . . . 46

5.2 Intra-slice isolation simulation parameters $\ldots \ldots \ldots \ldots$

6.1 Description of variables for secure slicing optimization model . . . . . 67

6.2 DDoS Experiment Topology Hardware Specification . . . . . . . . . 72

6.3 Inter-slice isolation DDoS experiment parameters $\ldots \ldots \ldots$

6.4 Secure allocation performance evaluation parameters $\ldots \ldots \ldots$

7.1 Simulation Parameters . . . . . . . . . . . . . . . . . . 93

8.1 Example Slice CPU Requests Database . . . . . . . . . . . . . 98

8.2 Example Slice CPU Allocations Database . . . . . . . . . . . . . 98

8.3 Experiment Topology Hardware Specification _ . . . . . . . . . . 102

8.4 Total Available Resources for Allocation Across All Servers . . . . . . 103

8.5 Slice Request Parameters . . . . . . . . . . . . . . . . . . . . . 103

8.6 Experiment Scenarios . . . . . . . . . . . . . . . . . . . . . 104 


\section{List of Figures}

2.1 A simplified comparison between tractional network and SoftwareDefined Networking (SDN) Architecture . . . . . . . . . . . 11

2.2 High-level overview of the LTE architecture . . . . . . . . . . . . . 14

2.3 Fifth Generation (5G) System architecture in reference point representation. N 1-22 are the reference points between VNFs . . . . . . . . . 16

2.4 Logical view of network slicing in 5G Networks. 5G core is virtualized and the VNFs of slice\#1, slice\#2 and slice\#3 can be hosted on one or more physical servers. . . . . . . . . . . . . . . . . . . . 18

2.5 Network Slice Selection Procedure . . . . . . . . . . . . . . . . . 19

4.1 Relationship between $T_{\text {HardTimeout }}, \lambda_{n}$ and $n \ldots \ldots$. . . . . . 32

4.2 Logical Topology . . . . . . . . . . . . . . . . . . 35

4.3 Ratio of completed and timed out requests . . . . . . . . . . . . 38

4.4 Response time comparison for constant rate DDoS . . . . . . . . . . 39

4.5 Load vs. Response time vs. Number of server . . . . . . . . . . . . . 40

5.1 An example of Network Slicing - A RAN slice can be connected with one or more core slice and vice versa. The slice pairing function is used to connect the RAN-core slice pair. . . . . . . . . . . . . 
5.2 Simulation topology. S1-S200, E1-E10, A1-A4, and DC1-DC2 represent physical servers, Edge, Aggregation and Datacenter switches, respectively . . . . . . . . . . . . . . . . . . 50 50

5.3 CPU utilization, bandwidth utilization and requests accepted for vary-

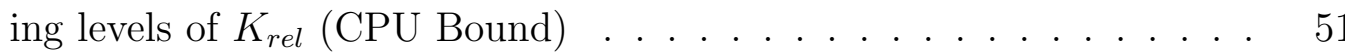

5.4 CPU utilization, bandwidth utilization and requests accepted for varying levels of $K_{r e l}$ (Bandwidth Bound) . . . . . . . . . . . . . . . . . . 52

5.5 Average solver runtime (seconds) for varying levels of $K_{r e l} \quad \ldots \ldots$

5.6 CPU utilization for different end-to-end delay requirements . . . . . . 53

5.7 Bandwidth utilization for different end-to-end delay requirements . . 55

5.8 Average solver runtime (seconds) for different end-to-end delay require-

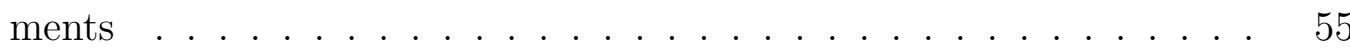

6.1 Logical view of DDoS attack on a slice . . . . . . . . . . . . 63

6.2 Evaluation Setup. The components used in the ns3 simulation are UE, Evolved NodeBs (eNB), Mobility Management Entity (MME), Packet Data Gateway (PGW) and $\mathbf{C}$ is a traffic trace collection node. PS1PS5 are the slice allocation servers, and A1 is a DDoS node. TF (pcap) is the traffic trace file collected in the simulation at $\mathbf{C}$ and replayed at A1 using tcpreplay ...................... . . 73

6.3 Flow diagram of the authentication procedure used in the experiments (V1, V2 and V3 represents virtual network functions) . . . . . . . 75

6.4 Response Time for flooding DDoS attack scenario . . . . . . . . . . 78

6.5 Round-Trip Time for flooding DDoS attack scenario . . . . . . . . . . 79

6.6 Average available bandwidth (BW) for the slice in flooding DDoS attack scenario . . . . . . . . . . . . . . . . . . 80

6.7 Response Time for slice-initiated attack scenario . . . . . . . . . . . . 81 
6.8 Round-Trip Time for slice-initiated attack scenario . . . . . . . . . . 82

6.9 Simulation Topology . . . . . . . . . . . . . . . . . . 84

6.10 Performance evaluation for varying level of $K_{r e l} \ldots \ldots$. . . . . . . . . 86

7.1 Comparison of Co-Residency Success rate for different average CPU

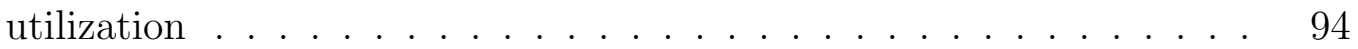

8.1 DSAF Logical Topology in our experiments: The brown boxes represent the physical servers and blue boxes represent the framework components. Solid lines show the logical communication paths between framework components . . . . . . . . . . . . . . . . . 100

8.2 Dynamic Slice Allocation Optimization Flow Diagram . . . . . . . . . 100

8.3 DSAF Physical Topology. All links are 1 Gbps . . . . . . . . . . 102

8.4 Comparison of Allocation Schemes for Different Scenarios . . . . . . . 105

8.5 Total Slice Requests Allocated . . . . . . . . . . . . . . . . . 106

8.6 Overhead: Processing Time . . . . . . . . . . . . . 106

8.7 Overhead: Average Computation Time Per Slice . . . . . . . . . . . 107 


\title{
List of Abbreviations
}

\author{
1G First Generation \\ 3G Third Generation \\ 5G Fifth Generation
}

ABB Adpative Bubble Burst

AF Application Function

AMF Access and Mobility Management Function

APIs Application Programming Interfaces

ARPANET Advanced Research Projects Agency Network

AUSF Authentication Server Function

CCNF Common Control Network Functions

CCP Common Control Plane

CDMA Code-Division Multiple Access

CDN Content Delivery Network

CN Core Network 
CPU Central Processing Unit

DDoS Distributed Denial-of-Service

DMZ Demilitarized Zone

DNS Domain Name Servers

DoS Denial-of-Service

DSAF Dynamic Slice Allocation Framework

EDGE Enhanced Data rates for Global Evolution

eNB Evolved NodeBs

EPC Evloved Packet Core

ETSI European Telecom Standards Institute

FCFSFA First Come First Serve First Available

FDDI Fiber Distributed Data Interface

FQDN Fully Qualified Domain Name

FTP File Transfer Protocol

GA Greedy Algorithm

GPRS General Packet Radio Service

GSM Global System for Mobile communications

HDD Hard Disk Drive 
HSPA High Speed Packet Access

HTTP HyperText Transfer Protocol

IMS IP Multimedia Subsystem

IP Internet Protocol

ISI Inter-Slice Isolation

LAN Local Area Network

LP Linear Programming

LSI Large-Scale Integrated

LTE Long-Term Evolution

MANO Management and Orchestration

MEC Multi-access Edge Computing

MILP Mixed-Linear Integer Programming

MME Mobility Management Entity

MVNO Mobile Virtual Network Operator

NAS Non-Access Stratum

NFV Network Function Virtualization

NGMN Next Generation Mobile Networks

NSSAI Network Slice Selection Assistance Information 
NSSF Network Slice Selecton Function

NTP Network Time Protocol

OFRHM OpenFlow Random Host Mutation

PGW Packet Data Gateway

RAM Random Access Memory

RAN Radio Acess Network

RTT Round-Trip Time

SDN Software-Defined Networking

SEAF Security Anchor Function

SLA Service Level Agreements

SMF Session Management Function

TCP Transmission Control Protocol

UDM Unified Data Management

UDP User Datagram Protocol

UE User Equipment

UPFs User Plane Functions

VM Virtual Machine

WIFi Wireless Fidelity 


\title{
List of Symbols
}

\author{
$\lambda_{c} \quad$ Incoming traffic rate (packets/sec) \\ $\lambda_{s} \quad$ Traffic handled by each server (packets/sec) \\ $T_{\text {IdleTimeout }} \quad$ Flow idle time-out \\ $T_{\text {HardTimeout }}$ Flow hard time-out \\ $T_{\text {Max }} \quad$ Maximum timeout value used at $\lambda_{1}$ \\ $P_{d} \quad$ Drop probability \\ $g^{i} \quad$ CPU demand of VNF i in $\mathrm{GHz}$ \\ $g^{i j} \quad$ Bandwidth demand of the edge $(\mathrm{i}, \mathrm{j})$ in Mbps \\ $r_{u} \quad$ Remaining capacity of the server $\mathrm{u}$ in $\mathrm{GHz}$ \\ $r_{u, \max } \quad$ Maximum capacity of the server $\mathrm{u}$ in $\mathrm{GHz}$ \\ $r_{u v} \quad$ Remaining capacity of the link $(\mathrm{u}, \mathrm{v})$ in Mbps \\ $r_{u v, \max } \quad$ Maximum capacity of the link $(\mathrm{u}, \mathrm{v})$ in Mbps \\ $d_{u v} \quad$ Delay of the link $(\mathrm{u}, \mathrm{v})$ in $\mathrm{ms}$ \\ $\gamma_{u}^{i} \quad$ indicates, if VNF i can be allocated to server $\mathrm{u}$
}




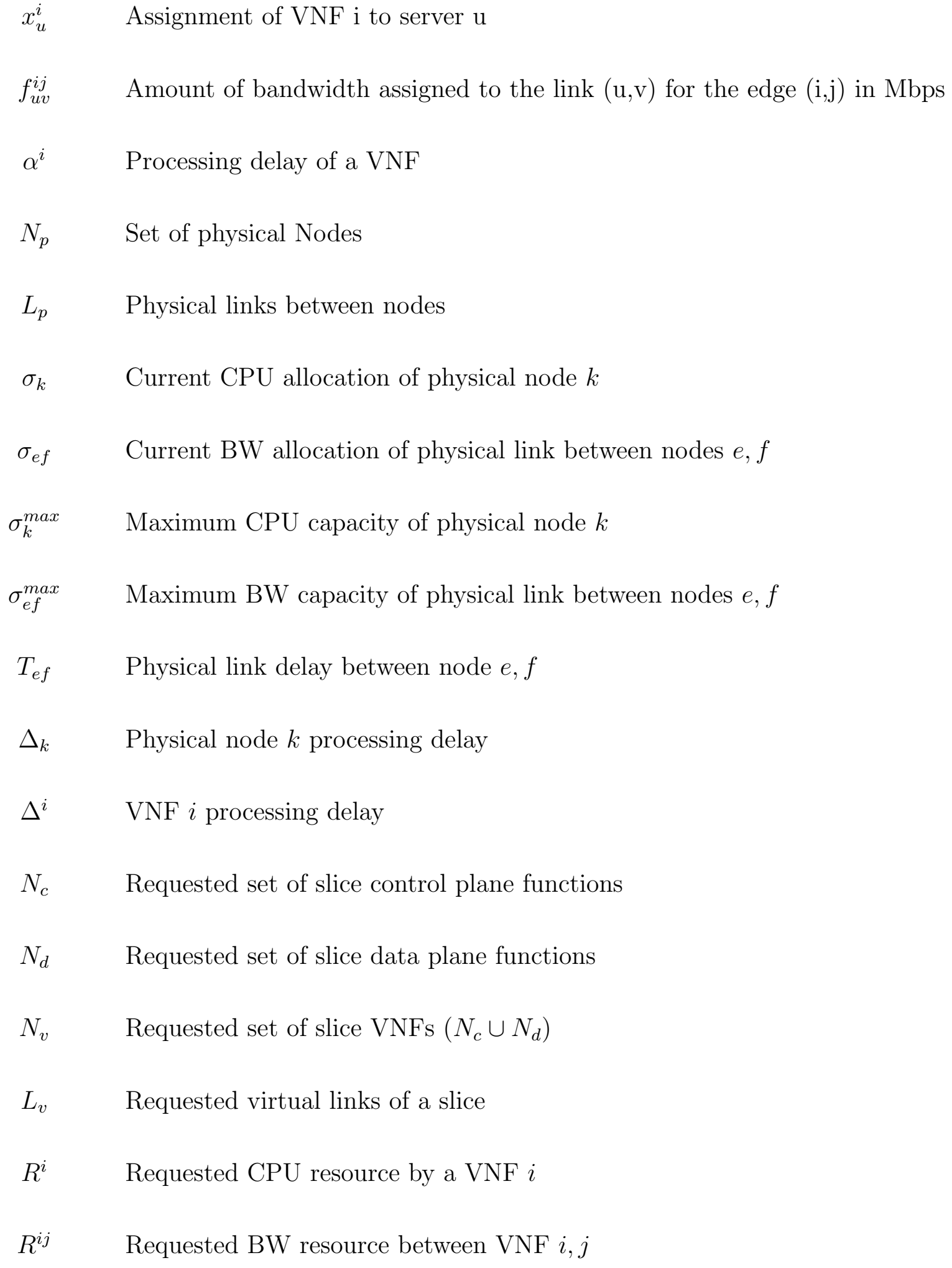




$\begin{array}{ll}d_{E 2 E} & \text { Requested End-to-End delay } \\ K_{r e l}^{c} & \text { Requested intra-slice isolation for Control Plane } \\ K_{r e l}^{d} & \text { Requested intra-slice isolation for Data Plane } \\ \gamma^{c} & \text { Requested inter-slice isolation for Control Plane } \\ \gamma^{d} & \text { Requested inter-slice isolation for Data Plane } \\ u_{k}^{i} & \text { Indicates the assignment of VNF } i \text { to EPC node } k \\ y_{e f}^{i j} & \text { Indicates the assignment of link }(e, f) \text { for VNF graph edge }(i, j)\end{array}$




\section{Chapter 1}

\section{Introduction}

66 There are no secrets better kept than the secrets that everybody guesses. 99

George Bernard Shaw

Virtualization is defined as a software technology that introduces an abstraction layer between the applications, operating systems, and hardware. This abstraction layer is called a hypervisor [1]. It hides the underlying hardware from the applications and operating systems. Since the hypervisor controls the hardware resources, it is possible to run multiple operating systems in parallel. There are several advantages of virtualization. It could offer flexibility, availability, scalability, efficient hardware utilization, a degree of security by providing some level of isolation, and cost-effectiveness. As there are many advantages of virtualization, there are also some disadvantages of virtualization, e.g., overhead, performance, single point of failure, and security of applications, to name a few [1].

A new concept called Network Function Virtualization (NFV) was proposed to take advantage of the virtualization, in which network functions will run on the commodity hardware. NFV has emerged as a new network architecture that could provide a flexible, agile, and cost-effective solution for the next generation networks, 
which accelerated the development and deployment of many different types of systems, e.g., Cloud Network, SDN, Content Delivery Network (CDN) and 5G Mobile Network. The European Telecom Standards Institute (ETSI) developed NFV framework. ETSI selected six use cases for NFV, i.e., NFV as a service, virtualization of mobile network and IP Multimedia Subsystem (IMS), virtualization of cellular base stations, virtualization of the home environment, virtualization of CDNs, and fixed access NFV [2].

Each type of virtualized network comes with a host of security issues. However, some of the common virtualization security issues are [2]:

- Communication between Virtual Machine (VM) or between host and VMs: In some cases, the virtualization layer logs keystrokes and screen updates stored on the host. It could be possible to snoop on the communication between the host and VMs or between the VMs.

- VM Escape: The hypervisor provides the isolation between the VM and the host. However, it might be possible to bypass the virtualization layer and access the host machine (it might be possible to gain root access as well).

- VM Monitoring from the Host: The host can modify the VMs (e.g., start, stop, change allocated resources). It can also copy and view the VM's virtual disks and snoop on the network traffic.

- Denial-of-Service: A Denial-of-Service (DoS) attack on one VM could impact other VMs. For instance, overwhelming the network link of the host machine would impact the hosted VMs.

- VM Resource Starvation: Another type of DoS attack is a resource starvation attack. In this kind of attack, a VM would try to exhaust the host resources 
(e.g., Central Processing Unit (CPU), Random Access Memory (RAM), or CPU cache) that could result in a DoS on other VMs.

In this dissertation, we note that our focus is only on Denial-of-Service and VM Resource Starvation attacks.

\subsection{Motivation}

Online service availability is a crucial part of many businesses. When a resource is under a Distributed Denial-of-Service (DDoS) attack, it can suffer from degradation in the quality of service, and in some scenarios, it could become completely unavailable. That might lead to information or financial loss. There are several existing methods to handle DDoS traffic. One method is to let the specific resource handle all the traffic, which could lead to resource unavailability. Another method is to filter all the illegitimate traffic at the gateway nodes using existing security solutions (e.g., firewall, intrusion detection systems, intrusion prevention systems, etc.) and only pass legitimate traffic to the specific resource. The latter method could add overhead in terms of processing and delay. This motivated us to further investigate the problem and find a new solution that could provide service availability while the attack is happening in a virtualized environment.

Another key motivation to present our work is that future mobile networks must provide services to a vast array of applications and devices with competing and perhaps conflicting requirements while simultaneously allowing flexible and agile deployment. The 5G networks deal with this issue in part by relying on network slicing, which has emerged as a key-enabler for proving heterogeneous services with different requirements to enable rapid and scalable deployment of the $5 \mathrm{G}$ network (applications). Network slicing takes advantage of virtualized infrastructures, where multiple 
services can be hosted on the same physical infrastructure, while at the same time guaranteeing Service Level Agreements (SLA), therefore, allowing flexible and efficient utilization of limited resources.

A shift in the mobile network paradigm also mandates changes in the security architecture with new security challenges that do not exist in the past or present mobile networks [3]. In the current mobile network, if an attacker launches a flooding Distributed Denial of Service (DDoS) attack against a mobile service, only that service will be affected. However, in a $5 \mathrm{G}$ network, a DDoS against one slice might affect other services because they could be tenants of the same shared virtualized infrastructure.

\section{$1.2 \quad$ Scope}

The objective of our work is not to detect or stop the DDoS attack but to maintain service availability during the attack and reduce the impact. Our proposed solutions are not a replacement for the existing DDoS defenses (e.g., Intrusion detection/prevention systems, firewalls, etc.). However, they can be deployed in conjunction with them. Our work in the $5 \mathrm{G}$ mobile network is focused on the security of network slicing only. More specifically, on 5G core network slicing using isolation.

\subsection{Research Question}

Distributed Denial-of-service attacks are difficult to mitigate. During a flooding DDoS attack, the attacker will try to overwhelm the target network or service by sending a large amount of traffic. The challenge is to keep the target network or service available to the end-users. In a virtualized network, multiple tenants share the same physical resources. A DDoS attack on one tenant could disrupt the services for other 
tenants. There could be two attack vectors that can be used to affect other tenants. If the communication link is shared between multiple tenants, then a DDoS attack on one tenant could impact other tenants. The other attack vector would be if multiple tenants are hosted on the same host, then the attacker can take advantage of the cohosting (co-residency) and launch an attack on other tenants. For instance, increased Central Processing Unit (CPU) utilization of service could have an adverse effect on the performance and availability of other services.

In this research, we try to answer the following questions:

- How to increase availability of a service under DDoS in the SDN and the 5G Network?

- Can resource management be used to mitigate DDoS attacks in the 5G mobile network?

- Can resource management provide any defense against malicious co-residency?

\subsection{Contributions}

- DDoS Mitigation in SDN using Load-Balance (Chapter 4) : The availability of online services is threatened by the Distributed Denial of Service (DDoS) attacks. As computing resources are getting cheaper, DDoS attacks are becoming more frequent and powerful. When DDoS attacks are directed towards a specific service, a small number of bots can overwhelm the service, making it unavailable to legitimate users. In this chapter, we present the Adaptive Bubble Burst (ABB), which takes advantage of the logically centralized architecture of Software-Defined Networks to provide mitigation against DDoS attacks that are targeted towards a specific service. ABB works by dispersing 
the incoming traffic in the network. Our experimental results show that ABB mitigates the DDoS attacks by enhancing the availability of a specific network resource.

- Slice Isolation using Optimal Resource Allocation (Chapter 5): 5G network slicing is essential to providing flexible, scalable, and on-demand solutions for the vast array of applications in 5G networks. Two key challenges of $5 \mathrm{G}$ network slicing are function isolation (intra-slice) and guaranteeing endto-end delay for a slice. In this chapter, we address the question of optimal allocation of a slice in $5 \mathrm{G}$ core networks by tackling these two challenges.

- DDoS Mitigation using Slice Isolation in 5G Networks (Chapter 6): In this chapter, we present our work to proactively mitigate Distributed Denial-ofService attacks in 5G core network slicing using slice isolation. Network slicing is one of the key technologies that allow $5 \mathrm{G}$ networks to offer dedicated resources to different industries (services). However, a Distributed Denial-of-Service attack could severely impact the slices' performance and availability as they could share the same physical resources in a multi-tenant virtualized networking infrastructure. Slice isolation is an essential requirement for $5 \mathrm{G}$ network slicing. We use slice isolation to tackle the challenging problem of Distributed Denialof-Service attacks in $5 \mathrm{G}$ network slicing. We utilize a mathematical model that can provide on-demand inter and intra-slice isolation for $5 \mathrm{G}$ core network slices.

- Defense against Malicious Co-residency (Chapter 7): Malicious coresidency in virtualized networks poses a real threat. The next-generation mobile networks heavily rely on virtualized infrastructure, and network slicing has emerged as a key enabler to support different virtualized services and applications in the $5 \mathrm{G}$ network. However, allocating network slices efficiently 
while providing a minimum guaranteed level of service and defense against the malicious co-residency threat in a mobile core is challenging. In this chapter, we analyze the defense against the malicious co-residency using our optimizationbased allocation to address this question.

- Dynamic Slice Allocation Framework (Chapter 8): In this chapter, we extend our work to allocate slices dynamically. We propose a dynamic slice allocation framework for the $5 \mathrm{G}$ core network. The proposed framework automates the process of slice allocation and deallocation. It can also provide on-demand user-interaction to accept single or multiple requests.

\subsection{List of Publications}

\subsubsection{Publications included in the thesis}

1. Danish Sattar, Ashraf Matrawy and Olu Adeojo, "Adpative Bubble Burst (ABB): Mitigating DDoS attacks in Software-Defined Networks," in 2016 17th International Telecommunications Network Strategy and Planning Symposium (Networks), Sep. 2016, pp. 5055.

2. Danish Sattar, Ashraf Matrawy, "Optimal Slice Allocation in 5G Core Networks," IEEE Networking Letters, pp. 11, 2019

3. Danish Sattar, Ashraf Matrawy, "Towards Secure Slicing: Using Slice Isolation to Mitigate DDoS Attacks on 5G Core Network Slices", in Proc. of the 7th Annual IEEE Conference on Communications and Network Security (CNS 2019), June 2019.

4. Danish Sattar, Ashraf Matrawy, "Secure 5G Core Network Slicing for DDoS 
Mitigation", under review.

5. Danish Sattar and Ashraf Matrawy, "Proactive and Dynamic Slice Allocation in Sliced 5G Core Networks," published in IEEE International Symposium on Networks, Computers and Communications, October 2020

\subsubsection{Publications not included in the thesis}

1. Danish Sattar, Ashraf Matrawy, T. Bryant, and M. Kneppers, "Threat Modeling in LTE Small Cell Networks," in 2018 Canadian Conference on Electrical 86 Computer Engineering (CCECE). IEEE, 2018, pp. 15.

2. Danish Sattar, Ashraf Matrawy, "An empirical model of packet processing delay of the Open vSwitch," IEEE 25th International Conference on Network Protocols (ICNP), 2017, pp. 1-6. 


\section{Chapter 2}

\section{Background}

6 Security in IT is like locking your house or car - it doesn't stop the bad guys, but if it's good enough they may move on to an easier target. 99

Paul Herbka

\section{$2.1 \quad$ Evolution of Networks}

\subsubsection{Computer Networks}

The earliest global connection was established between computers in the late 1960s (batch-processing networks). Batch-processing systems were powerful and reliable computers and were based on mainframes. Advanced Research Projects Agency Network (ARPANET) was one of the first computer networks that used packet switching, and it was established in 1969. In the early 1970s, the arrival of Large-Scale Integrated (LSI) circuits led to the first minicomputer and the development of the first propriety Local Area Network (LAN). In the first LAN, there were different types of proprietary interfaces and various methods of proprietary data representations. However, the invention of Transmission Control Protocol (TCP)/Internet Protocol (IP) 
in the late 1970 s and the installation of TCP/IP protocol stack on all nodes in the early 1980s led to the creation of the Internet in its current form. The next major milestone was the development of the first LAN standard technologies in the 1980s (i.e., Ethernet, Token Ring, and Fiber Distributed Data Interface (FDDI)) [4]. Ethernet standard became the most dominant LAN technology. Currently, the Ethernet speeds can reach up to 100 Gigabit/Sec.

The traditional network devices are usually built using proprietary hardware and software, and they also require proprietary software to manage them. However, the wide-spread adaption of virtualization and NFV has led to a revolutionary way to manage the networks and network devices. SDN architecture decouples the control and data plane as well as allows the programming of the control plane. The concepts of decoupling of control and data plane and programmability are not entirely new and have been proposed previously but as separate features in different protocols and software [5]. The uniqueness of SDN resides on the fact that it provides simple and easy to program through the decoupling of control and data plane as well as less complexity for network devices. SDN decouples the forwarding and network control that provides an abstraction from underlying infrastructure while simultaneously allowing the programmability of the control plane. Another feature of SDN is, it maintains the global view of the network, which could be used for faster reaction to network changes.

SDN architecture can be divided into three functional layers: an application layer, a control layer, and an infrastructure layer [5]. The application layer mainly consists of SDN applications designed to fulfill end-user requirements. An example of an end-user application would be security applications. The control plane is the brain of the SDN network. It contains the controllers and provides consolidated control functionality to supervise the Infrastructure layer and Application Programming Interfaces (APIs) 
for the application layer. It uses the south-bound interface to communicate with the infrastructure layer and north-bound interface to communicate with the application layer. The infrastructure layer contains the forwarding agents (e.g., switches, routers) in the data plane. This layer is responsible for collecting network information and forwarding traffic according to the rules provided by the control layer. Fig. 2.1 shows a simplified network architecture comparison between traditional networks and SDN.

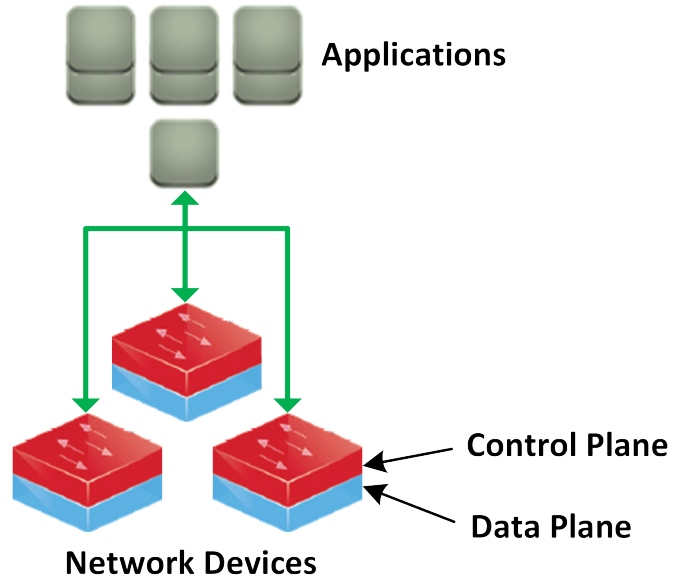

(a) Traditional Network
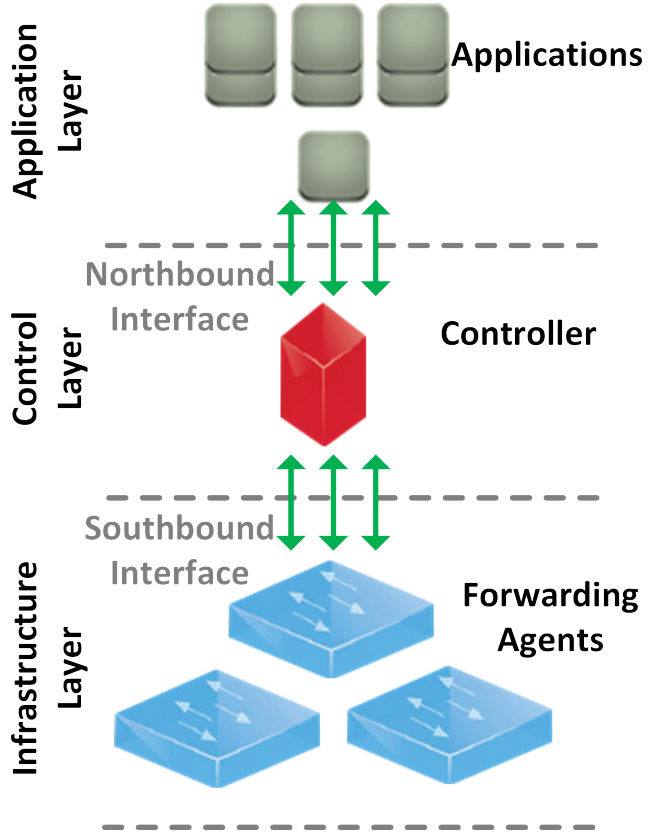

(b) SDN Architecture

Figure 2.1: A simplified comparison between tractional network and SDN Architecture

\subsubsection{Mobile Networks}

In this section, we discuss the evolution of the mobile network, but the primary focus will be the core network and data technologies. 
The First Generation (1G) of mobile networks were deployed in 1984. These were analog systems with limited usage because each nation developed and deployed its system with no data connectivity. Global System for Mobile communications (GSM) was proposed as a successor to the $1 \mathrm{G}$ system, and it began replacing $1 \mathrm{G}$ networks in the early 1990s. However, it took almost a decade for the first data technology to be deployed in a mobile network. The first data network deployed in the mobile network was General Packet Radio Service (GPRS) and later on Enhanced Data rates for Global Evolution (EDGE) that improved data speeds. However, the radio and core networks remained analog [6]. The Third Generation (3G) brought improvements to both voice and data networks. There were several data technologies improvement over the life span of 3G networks (i.e., Evolved EDGE, Code-Division Multiple Access (CDMA) 2000, High Speed Packet Access (HSPA), and HSPA+) and overtime network functions of the core network started to use packet switching for a vast majority of communication. The major shift came with Long-Term Evolution (LTE) network. The end-to-end communication (Radio Acess Network (RAN) and Core network) used IP communication that brought significant improvements to the voice quality and increased data speeds [6]. However, the entire mobile network still used bare-metal systems (purpose build components), but with the arrival of $5 \mathrm{G}$ networks, that is about to change. The $5 \mathrm{G}$ network takes advantage of NFV in both RAN and core network. It will be using soft-RAN, and core functions will be virtualized to fulfill the demands of the ever-growing need for mobile services. Table 2.1 shows an overview of the evolution of mobile networks. 
Table 2.1: Evolution of Mobile Networks

\begin{tabular}{c|c|c|c|c|c}
\hline & $\mathbf{1 G}$ & $\mathbf{2 G}$ & $\mathbf{3 G}$ & $\mathbf{4 G}$ & $\mathbf{5 G}$ \\
\hline Deployment & 1980 & 1991 & 2002 & 2009 & 2020 \\
\hline Core Network & PSTN & PSTN & PSTN, Packet & Packet & Packet \\
\hline Data Technology & Analog & GPRS, EDGE & $\begin{array}{c}\text { Evolved EDGE, CDMA2000, } \\
\text { HSPA, HSPA+ }\end{array}$ & $\begin{array}{c}\text { WiMAX, LTE, } \\
\text { LTE-A, LTE-A Pro }\end{array}$ & $5 \mathrm{5G}$ \\
\hline Data Bandwidth (Up to) & - & 1 Mbps & 21 Mbps & 3 Gbps & 10 Gbps \\
\hline Core Network Infrastructure & Bare-metal & Bare-metal & Bare-metal & Bare-metal & Virtualized \\
\hline \hline
\end{tabular}

\subsection{LTE Network}

LTE networks were a major shift in the paradigm of mobile networks. Mobile networks shifted from a combination of circuit and packet switching to an all IP network and improved voice quality and data speeds. Another major improvement was latency for time-critical applications and increased cell size with support for small cells. The Evloved Packet Core (EPC) used packet switching to route packets from end devices that are either using IPv4 or IPv6. Unlike previous mobile networks, LTE EPC provided always-on connectivity to the devices. In previous mobile networks, the IP connection was established on-request and teared-down after the session is complete [7].

In LTE networks, it was expected that a higher number of Small Cells (low-cost base-stations such as femtocells and picocells) would be present in ISP networks. The main driving force behind the growing use of the small cells is the rise of the number of Internet-connected mobile devices, leading to a significant increase in traffic load on cellular networks in general, including the LTE network [8]. The traditional base station (eNodeB) capabilities are not enough to cover a densely populated area or to provide complete indoor coverage inside a building. Network operators use small cells and Wireless Fidelity (WIFi) access points to offload the traffic [9]. The small cell (which may have built-in WiFi capabilities) have multifold benefits. The small cell 
can be deployed quickly and require little configurations at the customer's end. A small cell also provides extended coverage (i.e., indoors, in densely populated areas) [10] as well as the flexibility to tailor the services to each customer or a small area [11]. Fig. 2.2 shows a high-level view of the LTE architecture.

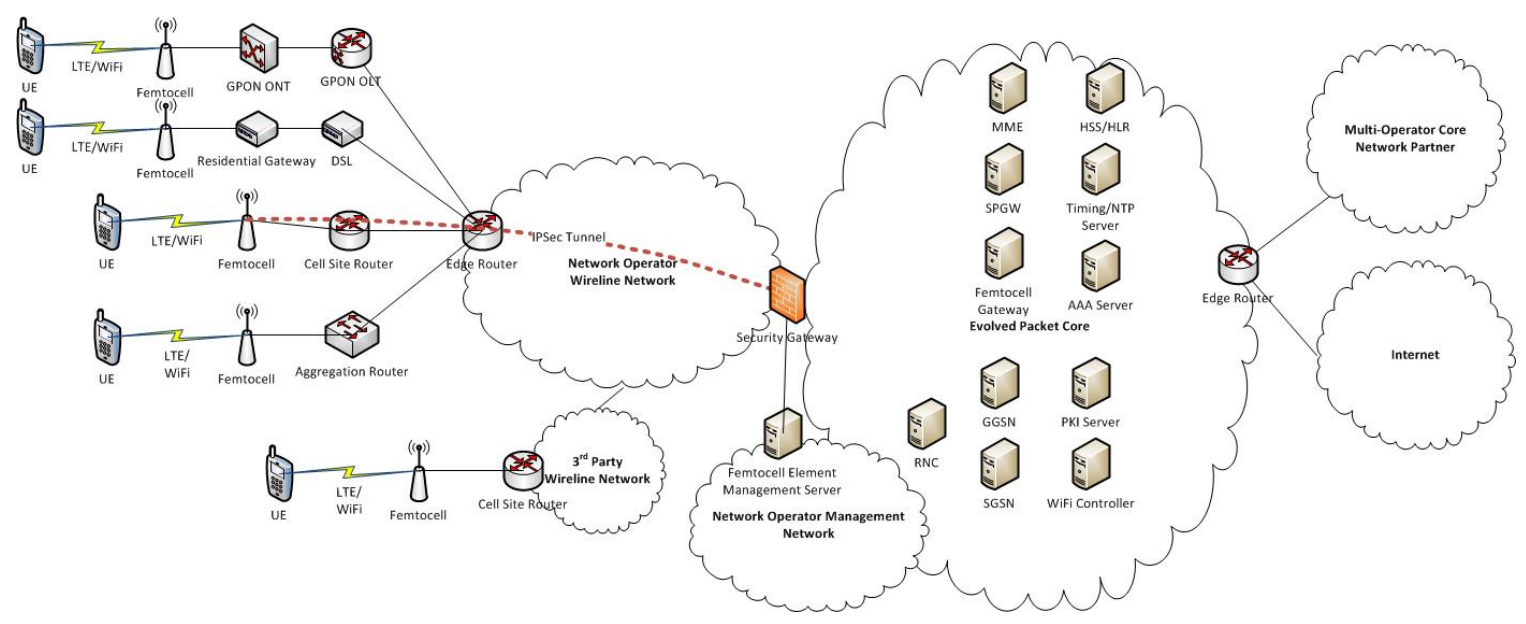

Figure 2.2: High-level overview of the LTE architecture

\section{$2.3 \quad 5 \mathrm{G}$ Network}

Current mobile networks are static and highly centralized. With ever-growing data volume, elastic demand for resources, and an agile application deployment cycle, it has been challenging to meet these demands in the current mobile network architecture. It is expected that in the near future, there will be a 1000-fold increase in data volume and 100-fold in connected devices [12]. The $5^{\text {th }}$ generation (5G) of the mobile network has been proposed to overcome the shortcomings of the current mobile networks and meet future demands. It must address six challenges that are not adequately addressed by the LTE network [13].

1. Higher traffic capacity 
2. Higher data rate

3. Low end-to-end latency

4. Massive data connectivity

5. Cost reduction and

6. Consistent Quality of Experience Provisioning

Eight requirements were set forth for the $5 \mathrm{G}$ network to address these challenges. [14].

1. 1000x bandwidth per unit area

2. $\sim 10 \mathrm{Gbps}$ data rate

3. $<1 \mathrm{~ms}$ of end-to-end latency (i.e., from user to the service)

4. 10-100x number of connected devices

5. $90 \%$ reduction in network energy usage

6. $100 \%$ network coverage

7. $99.999 \%$ availability

8. Up to 10-year battery life for low powered IoT devices.

The $5 \mathrm{G}$ network is envisioned to be an agile and elastic network. By leveraging virtualization and programmable networks, it will provide scalable and on-demand deployment of applications and network services. The 5G network uses millimeterwavelength for higher bandwidth. It might be required to integrate third-party deployments and multi-operator deployments to make it more economical and costeffective [13]. 5G is enabling new verticals, new services, and new business models 
that are not possible or practical with $4 \mathrm{G}$ and other legacy mobile technologies. Examples include wearables for advanced telemedicine applications, virtual/augmented reality, Ultra High Definition video, and machine-to-machine (M2M) applications that require single-digit-millisecond latencies such as driverless cars. 5G technologies aim to provide an end-to-end infrastructure capable of delivering a consistent user quality of experience in a heterogeneous environment across a wide variety of use cases. To achieve this goal, 5G must be able to support very high bit rates, high vehicular speeds, low latencies, and high device densities. One example is Internet of Things (IoT) applications, where there may be thousands of $5 \mathrm{G}$ devices within a single acre, all requiring single-digit-millisecond latencies and other capabilities that legacy mobile technologies cannot support well or at all [15]. Fig. 2.3 shows an overview of the 5G architecture [16].

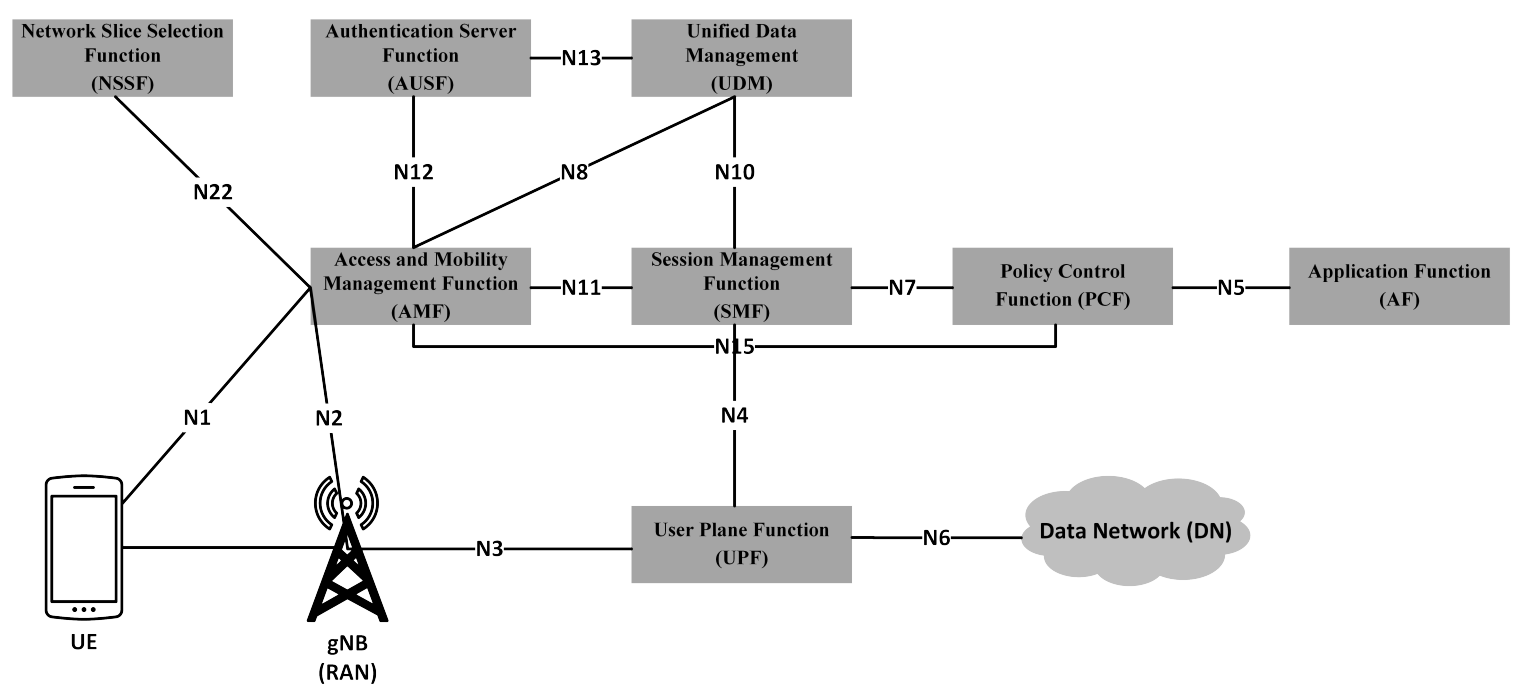

Figure 2.3: 5G System architecture in reference point representation. N 1-22 are the reference points between VNFs 


\subsubsection{G Network Slicing}

Faced with distinct and challenging service requirements, Network Slicing was proposed. The $5 \mathrm{G}$ network would fulfill fine-grained requirements for different services by slicing the physical network into smaller logical networks. A network slice is defined as:

A network slice is a complete and independent end-to-end logical network, including RAN and Core Network (CN). A network slice could be made using a single or multiple RAN and core slices. Network slices would share the same physical infrastructure; however, each network slice could be instantiated using different performance and security parameters. For instance, a customer can request a RAN and a core slice from the slice provider to create a mobile virtual network. In an ideal scenario, a customer would be able to request slices from multiple slice providers to create a cost-effective solution.

Network slicing leverages the latest innovations in cloud mobile access and core. Combining cloud technologies with the capabilities of software-defined networking (SDN) and network function virtualization (NFV) provides the necessary tools to enable network slicing. Virtualization technologies provide a key foundation for network slicing by enabling the use of both physical and virtual resources to create the service they are designed for. It is envisioned that this trend will continue, where virtualization technologies will be applied across RAN and portable services to wearable devices [15].

Network slicing provides many benefits, but the network slice operator needs to ensure that slices are isolated from each other to guarantee SLA. Networks slicing could have two different types of isolation, i.e., intra-slice isolation and inter-slice isolation. In our work, intra-slice isolation is defined as the physical separation of resources between the network functions of a slice, and inter-slice isolation is defined 
as the physical separation of resources between the slices.

In a $5 \mathrm{G}$ network, a slice could be instantiated for a specific service. Fig. 2.4 shows an example of different slices for autonomous vehicles (with a very low end-to-end delay requirement), high-speed mobile broadband (with high bandwidth requirements), or a dedicated slice for emergency services. In Fig 2.4, the Common Control Network Functions (CCNF) are shared among all three slices, and the Common Control Plane $(\mathrm{CCP})$ is only providing services to slice\#1 and slice\#2.

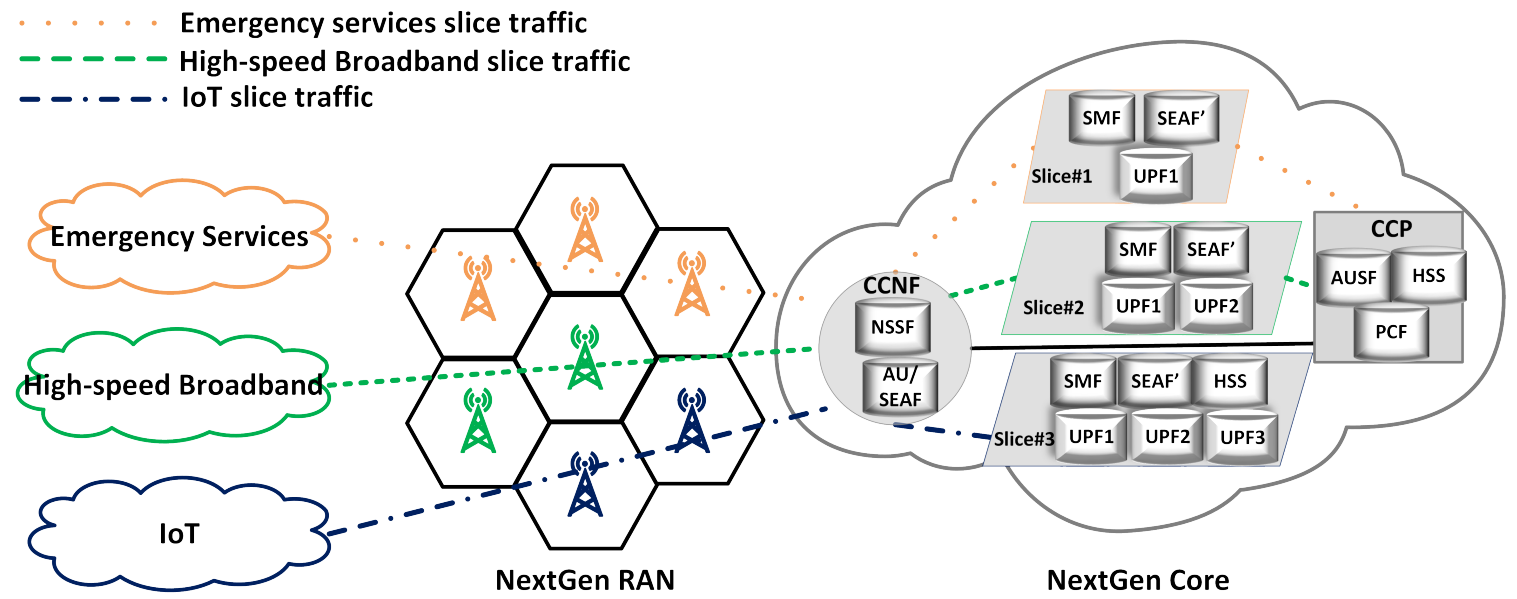

Figure 2.4: Logical view of network slicing in 5G Networks. 5G core is virtualized and the VNFs of slice\#1, slice\#2 and slice\# 3 can be hosted on one or more physical servers.

Each mobile operator (slice operator) could host multiple slices, and it would be essential to direct users to the correct slices. The Network slice selection and registration procedure is shown in Fig. 2.5. The mapping of RAN slice to the core slice is determined using the following procedure.

1. User Equipment (UE) sends a registration request to the mobile network via Access and Mobility Management Function (AMF). The message may include the Network Slice Selection Assistance Information (NSSAI) 
2. The request is directed to the AMF in CCNF. AMF retrieves data from Unified Data Management (UDM).

3. UDM responds with the subscription data to AMF. It would include SingleNSSAI (S-NSSAI)

4. AMF sends a request to Network Slice Selecton Function (NSSF) to select the appropriate slice for the UE.

5. NSSF determines the allowed NSSAI and selects the target AMF (it might respond with a list of AMF IP addresses or Fully Qualified Domain Name $(\mathrm{FQDN}))$.

6. AMF redirects the request to the target AMF

7. RAN sends the registration request to the target AMF and Non-Access Stratum (NAS) messages are exchanged, and a connection is established

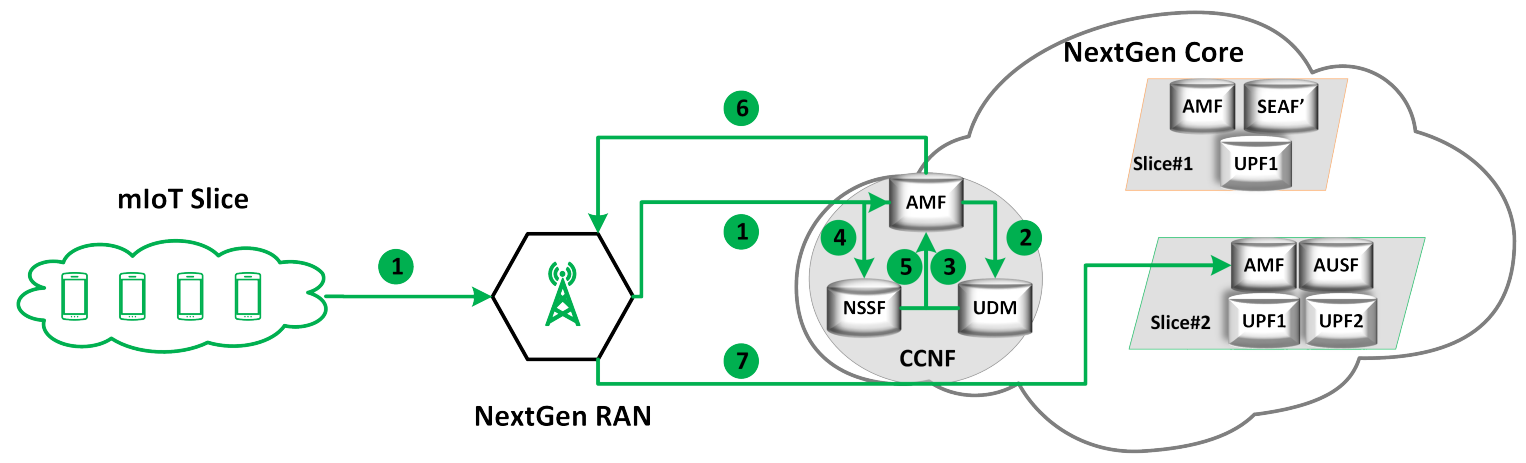

Figure 2.5: Network Slice Selection Procedure 


\section{Chapter 3}

\section{Research Problem and Methodology}

66The only truly secure system is one that is powered off, cast in a block of concrete and sealed in a lead-lined room with armed guards. 99

Gene Spafford

\subsection{Research Problem}

The Distributed Denial-of-Service attack is an attempt to disrupt an online service. Such an attack could have devastating consequences, especially in today's pandemichit economy, where a significant portion of businesses have moved to provide services online, and employees are working remotely. For instance, disrupting any video conferencing services like Zoom [17], Microsoft Teams [18], or Google Meet [19] would not only impact the service provider, but it will also prove devastating for other businesses that rely on these online services to conduct business.

Broadly DDoS attacks can have three categories. 1) Attacks that exploit some vulnerability or an implementation bug of the target service to cause disruption, 2) Attacks that exhaust the available resource of the target system/machine/service, and 3) Attacks that consume all the bandwidth available to the target service [20]. 
Network Function Virtualization has opened new avenues for providing services by decoupling software and hardware. It enables flexible and elastic deployment of network functions and application services. NFV is not only an important factor in the evolution of traditional computer networks but in the cellular networks as well. However, such flexibility comes at the cost of an increased threat surface. DoS attacks where host service/machine resources can be exhausted were relatively less common (as compared to the bandwidth exhaustion attacks) in traditional networks. VNFs are deployed on a shared physical infrastructure, where it is possible to be hosted on the same machine as the target service and exploit this proximity to perform resource exhaustion attacks. Similarly, bandwidth exhaustion DDoS attack on one service could lead to disruption in service for multiple applications due to the shared network link.

The security issues of the virtualized network functions were not as prevalent in mobile networks. However, this is changing with 5G networks. In 5G networks, applications can be deployed with their customized and unique requirements by employing network slicing. Network Slicing logically creates a specialized network on the shared physical infrastructure to cater to the application's needs. Such logical division also opens a way for the attacker to be hosted within the same physical infrastructure, which was not possible before ${ }^{1}$ in the mobile network. This could lead to different types of DDoS attacks on hosted services or the infrastructure.

This research aims to address the following questions.

- How to increase availability of a service under DDoS in the SDN and the 5G Network?

\footnotetext{
${ }^{1}$ Contractors, sub-contractors, or Mobile Virtual Network Operator (MVNO) under the control of parent telecommunication companies, e.g., Kodoo (MVNO) and TELUS (parent company), are present in the current mobile networks. However, in 5G networks, other customers can also be hosted on the same infrastructure that are not affiliated with the telecommunication provider.
} 
- Can resource management be used to mitigate DDoS attacks in the 5G mobile network?

- Can resource management provide any defense against malicious co-residency?

\subsection{Research Methodology}

In this section, we discuss the research methodology of the dissertation. The research held with respect to this dissertation is applied and new. Although several previous academic research pieces exist regarding the mitigating DDoS attacks, we take a look at DDoS problems in a relatively new domain (i.e., virtualized network with a focus on 5G mobile networks and SDN) with a different threat surface as compared to the traditional network. As such, the proposed research took a form of new research but on an existing research subject.

\subsubsection{Primary Data and Tools}

Data used in this research is generated using MATLAB, and it is used for simulations and experiments for network slicing. Various tools are used for different parts of the research. For instance, MATLAB, AMPL, and CPLEX are used for mathematical modeling, data generation, and simulation. At the same time, Python is used to develop the framework and modules required in this research. OpenVZ is used for container management, and the POX controller is used for SDN management.

\subsubsection{Research Process}

The research process started by examining the state-of-art literature on DDoS attacks and mitigation. We identified that there is a need for DDoS mitigation techniques for 
network slicing in a mobile network. We also identified that optimized network slice allocation would be essential for a mobile network. After identifying the problem, we proposed a solution to solve the problem, and it was validated using a combination of simulation and experiments. 


\section{Chapter 4}

\section{Adaptive Bubble Burst (ABB): Mitigating DDoS Attacks in Software-Defined Networks}

66 A secure system is one that does what it is supposed to. 99

Eugene Spafford

Software-Defined Network (SDN) offers a potential security advantage by providing a centralized approach that offers a global view of the entire network, making it easy to perform network analysis for anomaly detection. If an attack is detected, the controller can rapidly deploy network-wide preemptive security policies (e.g., load balancing, filter traffic, etc.) [21] [22]. On the other hand, SDN's new paradigm has presented new security challenges. For instance, the centralized controller becomes a lucrative target for attackers. Another highly effective attack against centralized paradigms is Distributed Denial of Service (DDoS) attacks [21]. There are two broad categories of DDoS attacks. 1) Bandwidth exhaustion attacks, where the attacker sends a tremendous amount of traffic that overwhelms the target's bandwidth. Typically, these types of attacks are connectionless [23,24]. 2) Service exhaustion attacks, 
in this type of attack, the attacker targets a specific service or a protocol (e.g., TCP, HyperText Transfer Protocol (HTTP), File Transfer Protocol (FTP), Network Time Protocol (NTP), etc.). The attacker attempts to disrupt the service by sending a large number of requests. These are usually connection-oriented attacks [23]. In this work, we deal with the latter (service exhaustion) category.

In this chapter, we propose a new mitigation technique called Adaptive Bubble Burst (ABB) that could be used in addition to traditional packet filtering and other DDoS defenses. The objective of the Adaptive Bubble Burst is to provide some availability of a specific resource (e.g., web server, file server, etc.) during DDoS attacks. ABB provides two layers of security. ABB hides the identity of protected service by advertising multiple services using a single virtual IP address. Secondly, when the bubble of DDoS traffic is coming towards the specific resource, ABB will mitigate the DDoS attack by adaptively spreading it across multiple replicas of the resource in the network. Therefore, reducing the impact on the original specific resource. ABB does not attempt to detect or stop attacks. The attack traffic will still propagate in the network, but ABB would enhance the availability of the specific resource.

\subsection{Security challenges in Software-Defined Net- works}

In this section, we will briefly discuss the security challenges of SDN. Some of the security threats to SDN are not new. They already exist for traditional networks. For instance, DoS/DDoS attacks are not new or limited to SDN, but their effect is amplified. If a DDoS attack is launched on the controller from the southbound interface, its effect on the network will be much worse than an attack against a single 
node in the traditional network. On the other hand, if a successful controller spoofing attack is launched from the northbound interface, then practically the entire network is compromised [22].

SDN also presents some new and unique security challenges. Applications can dictate their networking requirements to the controller via northbound APIs. In response, the controller converts those requirements into flows and installs them into the switches. Any malicious application can overwrite past policies/rules and make the network vulnerable (e.g., overwrite the old security rule with a new rule: bypass the firewall). There is no security mechanism in place to stop this kind of policy modifications [22].

Another potential attack on SDN could be data leakage. An attacker can determine the action taken by the switch for a specific packet (drop, forward, or send it to the controller) by doing processing time analysis. Having discovered the action, the attacker can easily craft a packet that will be forwarded to the controller. It could lead to a DDoS attack on the SDN controller [21].

Misconfiguration of the policies could be an issue in SDN. These policies are updated continuously as new security threats are detected. If there is inconsistency in-network or security policies, it can open the network to potential vulnerabilities and attacks. Currently, there is no protection from such misconfiguration of policies in OpenFlow. Another concern for the SDN is system-level security. It should be able to provide network audits all the time (e.g., which devices are up or down, network state, etc.). It can be a challenging task. For example, OpenFlow supports a fail-secure mode for switches; if they are disconnected from the controller, they can choose to operate on their internal logic that might not be the desired behavior. Therefore, it is crucial to understand the switch's forwarding behavior during interruptions because in order to provide auditing and accountability, such information should be managed 
by the SDN [21].

\subsection{Related Work}

Jafarian et al. [25] proposed OpenFlow Random Host Mutation (OFRHM) to protect user's online privacy, where the IP address of the end host mutates frequently and randomly to provide anonymity. OFRHM utilizes the idea of virtual IP addresses, where a user is assigned one real static IP that is only known to authorize entities, but for the outside node, the SDN controller will assign a random virtual IP address that is known via the Domain Name Servers (DNS). Each time if the outside node needs to communicate with the inside node, it would have to obtain a new IP address via the hostname resolution server (i.e., DNS). The intermediate OpenFlow switches are responsible for real to virtual and virtual to real IP translation, whereas the SDN controller is responsible for installing new flows, the virtual IP address assigned to each host, and DNS updates. OFRHM only provides anonymity, whereas we are using SDN to provide anonymity and to mitigate DDoS attacks.

Several techniques have been proposed to combat DDoS in software-defined networks. For instance, Nhu-Ngoc Dao et al. proposed to analyze the frequency of the packets sent by each host during a specific period to determine whether it is a legitimate user or a bot [26]. In DDoS Blocking Application (DBA) [27], DDoS is mitigated by moving the target host to a new IP address. The target server and DBA are actively involved in DDoS detection and mitigation. The server actively monitors some predefined metric for possible DDoS attacks. If an attack is detected and server collapse is imminent, it will notify the DBA on a secure channel. DBA assigns a new IP address, and the server will start providing the service at a new IP address. Afterward, all new clients will be given a new IP address with some challenge form, 
e.g., CAPTCHA. This technique assumes that bots cannot understand the challenge form and will be blocked after a certain number of wrong tries. DBA requires server modification, whereas our proposed scheme is transparent to the client and server.

IP Fast Hopping is a DDoS prevention mechanism, where the IP of the protected server is changing according to a schedule just like frequency hopping [28]. In this technique, when the clients request a particular server using a hostname, they are given the authorization server's IP address instead of the requested server. Once successfully authorized, they will be redirected to IP Hopper Manager (IHM). IHM is responsible for IP Hopping and secure communication between servers and clients. Clients use a virtual IP address called initial IP to start communication with the server. IHM will also allocate and send a pool of IP addresses to the client for IP hopping during that session. The server will hop IP addresses according to the pool allocated by the manager, and the client will also change the destination IP address. This should make it difficult for the attacker to trace or attack the protected server. IP Fast Hopping would add a delay in communication between client and server because clients need to first communicate with the IHM, and it would also disrupt the connection once a new server IP address is assigned. ABB requires no changes to the client and server.

An agent-based framework for a cooperative SDN is presented in [29] to mitigate the DDoS attacks. In this technique, each SDN autonomous system (AS) uses an agent(s) to build a cooperative SDN and packet filtering at the source to mitigate DDoS attacks. If one of the AS is under DDoS attack, it requests the origin AS to either limit the flow-rate or block it. In addition to these actions, it can also inform the other SDN autonomous systems about the DDoS attack. 


\subsection{Threat Model}

The attacker could be inside or outside the network where ABB is deployed. It could be an individual user or a botnet. We assume that the network can handle DDoS traffic, and the attacker is trying to disrupt a specific service, e.g., HTTP, FTP, DNS, etc., not the entire network. We also assume that the attacker does not have the capabilities to overwhelm the target network (i.e., by sending more traffic than its WAN link/devices can handle), but it can overwhelm the target service. Adaptive Bubble Burst is designed to mitigate the impact of the DDoS attacks against a service when the network is operational. The DDoS attack will reach inside the network and could cause damage to the resources.

\subsection{Adaptive Bubble Burst (ABB)}

In this section, we describe the Adaptive Bubble Burst. The main objective of ABB is to enhance the availability of a specific resource during a DDoS attack. Once incoming traffic reaches a certain threshold (i.e., a specific resource is about to reach its serving limit), ABB will burst the bubble of the DDoS attack by spreading the traffic directed towards the specific resource across multiple nodes in the network. These nodes are replicas of the specific resource (e.g., HTTP server, FTP server, Database serves, etc.). Therefore, reducing the impact on a single node and enhancing the availability of the specific resource. ABB is not a replacement for the DDoS defense solutions (e.g., intrusion detection systems, intrusion prevention systems, etc.). The attack traffic will still reach inside the network, which might cause damage to the resources, but it would significantly increase the chances of service being operational by employing $\mathrm{ABB}$ as a DDoS mitigation mechanism. A major advantage of ABB apart from DDoS mitigation is that it does not require any modifications or software at the client or 
server end. It is completely transparent to both ends (i.e., client and server).

\subsubsection{ABB Overview}

We will measure incoming traffic rate $\lambda_{c}$ (packets/sec) at gateway switches destined for a specific resource $\alpha$. The SDN controller will take these measurements at regular time intervals. If $\lambda_{c}$ reaches a certain threshold, $\mathrm{ABB}$ will start spreading the traffic dynamically. Our algorithm spreads the traffic according to five parameters that are traffic threshold levels $\lambda_{n}$ (packets/sec), number of available nodes $N$ (includes $\alpha$ and its replicas), flow idle timeout $T_{\text {IdleTimeout }}$, flow hard timeout $T_{\text {HardTimeout }}$ and drop probability $P_{d}$. The rate $\lambda_{c}$ is used to select the number of nodes $(n)$ to spread the traffic and $T_{\text {IdleTimeout }}, T_{\text {HardTimeout }}$ are used to balance the traffic for $n$ number of nodes. The drop probability of $P_{d}$ is used to drop the traffic once the system exhaust all of its resources and cannot handle any more traffic. At that point, ABB will drop some traffic according to probability $P_{d}$, which could be aggressive (static) or dynamic. ABB may drop the traffic aggressively by setting $P_{d}$ high value or dynamically adjust the $P_{d}$ value according to $\lambda_{c}$.

For simplicity, we assume that each server can handle $\lambda_{s}$ (packets/sec). At any given level of $\lambda_{n}$, $\mathrm{ABB}$ is using $n$ servers that satisfy the following inequality.

$$
\lambda_{n-1} \leq \lambda_{c}<\lambda_{n}
$$

The traffic threshold level $\lambda_{n}$ is given by equation (4.1).

$$
\lambda_{n}=n * \lambda_{s} \text { where } n=1,2,3, \ldots, N
$$


Where $n$ represents the number of servers used at any given level. System can successfully handle at maximum $\lambda_{N}$ (packets/sec), which is given by equation (4.2).

$$
\lambda_{N}=N * \lambda_{s}
$$

\subsubsection{Traffic Balancing}

Two parameters $T_{\text {IdleTimeout }}$ and $T_{\text {HardTimeout }}$ are used to balance the traffic between available number of nodes $(n)$. Flow idle and hard timeout are very important. Flow idle timeout is the time (seconds), after which flow will be removed from the switches if no matching packets arrive during that interval. On the other hand, flow hard timeout is the time (seconds), after which flow will be removed regardless of whether a flow is active or not. If they are too short, a significant amount of traffic will come to the controller. On the other hand, if they are too long, then traffic cannot be adequately balanced.

To adaptively balance the incoming traffic (which may include DDoS traffic), we would need to force packets toward the controller for new flow installation after a change in $\lambda_{n}$. Flow timeout values are the function of maximum timeout $T_{M a x}$, and the nodes used $n$ at any given $\lambda_{n}$. Equation (4.3) calculates flow idle and hard timeout values.

$$
T_{n}=\left\lfloor\frac{T_{\text {Max }}}{n}\right\rfloor \text { seconds } \quad \text { where } n=1,2,3, \ldots, N
$$




$$
\begin{gathered}
T_{\text {IdleTimeout }}=\left\lfloor\frac{T_{n}}{2}\right\rfloor \text { seconds } \\
T_{\text {HardTimeout }}=T_{n} \text { seconds }
\end{gathered}
$$

Where $T_{\text {Max }}$ is the maximum timeout value used at $\lambda_{1}$. If the flow is idle for $T_{\text {IdleTimeout }}$, it will automatically be removed from the switches clearing the memory of the switch. Shorter $T_{\text {IdleTimeout }}$ values are used because we would like to remove unused flows as soon as possible to free up the memory. If the flowage reaches $T_{\text {HardTimeout }}$ even if the flow is being used, it will be removed to force packets toward the controller for new flow installation. Therefore, allowing balancing the traffic. We note that $T_{M a x}$, and $T_{\text {IdleTimeout }}$ are carefully selected after performing experiments.

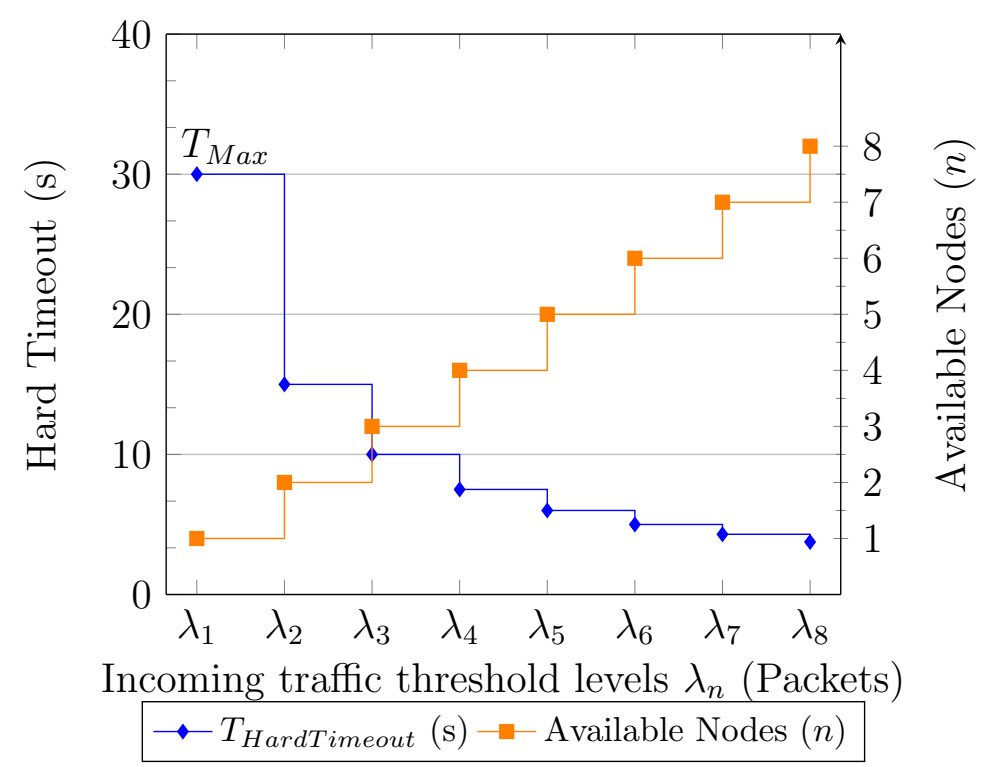

Figure 4.1: Relationship between $T_{\text {HardTimeout }}, \lambda_{n}$ and $n$

Fig. 4.1 shows an example of relationship between $T_{\text {HardTimeout }}, \lambda_{n}$ and $n . \lambda_{n}$ and $T_{\text {HardTimeout }}$ are calculated using equation (4.1) and (4.3), respectively. As $n$ increases, the hard timeout values decrease, which means more traffic will start going 
to the controller, thus starting the reactive load balancing of the incoming traffic. When $\lambda_{n}$ changes from a lower to a high value (e.g. $\lambda_{3}$ to $\lambda_{4}$ ), a reset signal is sent. Every switch that receives the reset signal will remove all the flows from its table. Therefore, forcing the incoming traffic to be redirected towards the controller. It will install new flows with maximum number of available nodes and short $T_{\text {IdleTimeout }}$ and $T_{\text {HardTimeout }}$. The reason for resetting the switches is that some previously installed flows could have much longer timeout values, and traffic might not be balanced. It would be undesirable to perform reset at a decreased value of $\lambda_{n}$ (e.g., $\lambda_{4}$ to $\lambda_{3}$ ) because it could lead to an unstable network as well as a loss of traffic. We note that $T_{\text {HardTimeout }}$ decreases rapidly for the first four levels, but later on, the change is not significant. It is also important to note that $T_{\text {IdleTimeout }}, T_{\text {HardTimeout }}$, and frequency of reset signals impact the amount of traffic going to the controller, which could affect the network stability. Further investigation is required as we did not test this in our experimentation. It would be critical to correctly configure these parameters to appropriately balance the traffic, as explained in this section.

\subsubsection{Traffic Drop}

Drop probability $P_{d}$ will come in effect once the system reaches maximum level $\lambda_{N}$ $\left(\lambda_{c}>N * \lambda_{s}\right)$. After this level, the ABB will begin dropping traffic according to $P_{d}$. If $P_{d}$ has a high value, the $\mathrm{ABB}$ will drop a considerable amount of traffic. On the other hand, we can control $P_{d}$ dynamically according to $\lambda_{c}$. The ABB does not distinguish between legitimate and DDoS traffic. $P_{d}$ is calculated using equation (4.4):

$$
P_{d}=1-\frac{\lambda_{s} * N}{\lambda_{c}}
$$

Equation (4.4) only generates positive values (i.e. $0 \leqslant P_{d} \leqslant 1$ ). 


\subsubsection{Server Anonymity}

In the literature, several techniques have been proposed to provide anonymity for the client or the server $[30,31]$. As noted in [25] that none of the existing techniques are transparently deployable to the client and the server. The techniques either require modification at the client or the server. We take advantage of the flexible programming environment provided by SDN, where a packet can be modified on-demand. We use a technique for server anonymity that is similar to OFRHM [25]. We use a virtual IP $(v I P)$ address for advertising multiple services outside the network where ABB runs. Real IP (rIP) addresses are hidden from the attacker and outside the network. The SDN controller will be responsible for instructing gateway switches to rewrite and redirect the incoming traffic according to the requested service. For instance, we are advertising three services FTP (20-21), web (80), and NTP (123) using vIP. Servers FTP, Web and NTP are hosted at $r I P_{1}, r I P_{2}$ and $r I P_{3}$, respectively. A user sends a request for time synchronization to the NTP server using $v I P$. The gateway switch will receive this request and forwards it to the controller. The controller will install two flows in the gateway switch. First, respond to any ARP request with the virtual MAC address and second, rewrite, and redirect the synchronization request to the NTP server, which is hosted at $r I P_{3}$ port 123. NTP server will send a reply accordingly, once the response packet reaches the gateway switch, the controller will install an outgoing flow in the gateway switch to rewrite the packet with $v I P$ and send it back to the user. Therefore hiding the real IP address of the specific server. Please note that server anonymity is a benefit of advertising multiple services on a single IP address. However, it is not the main focus of our work. 


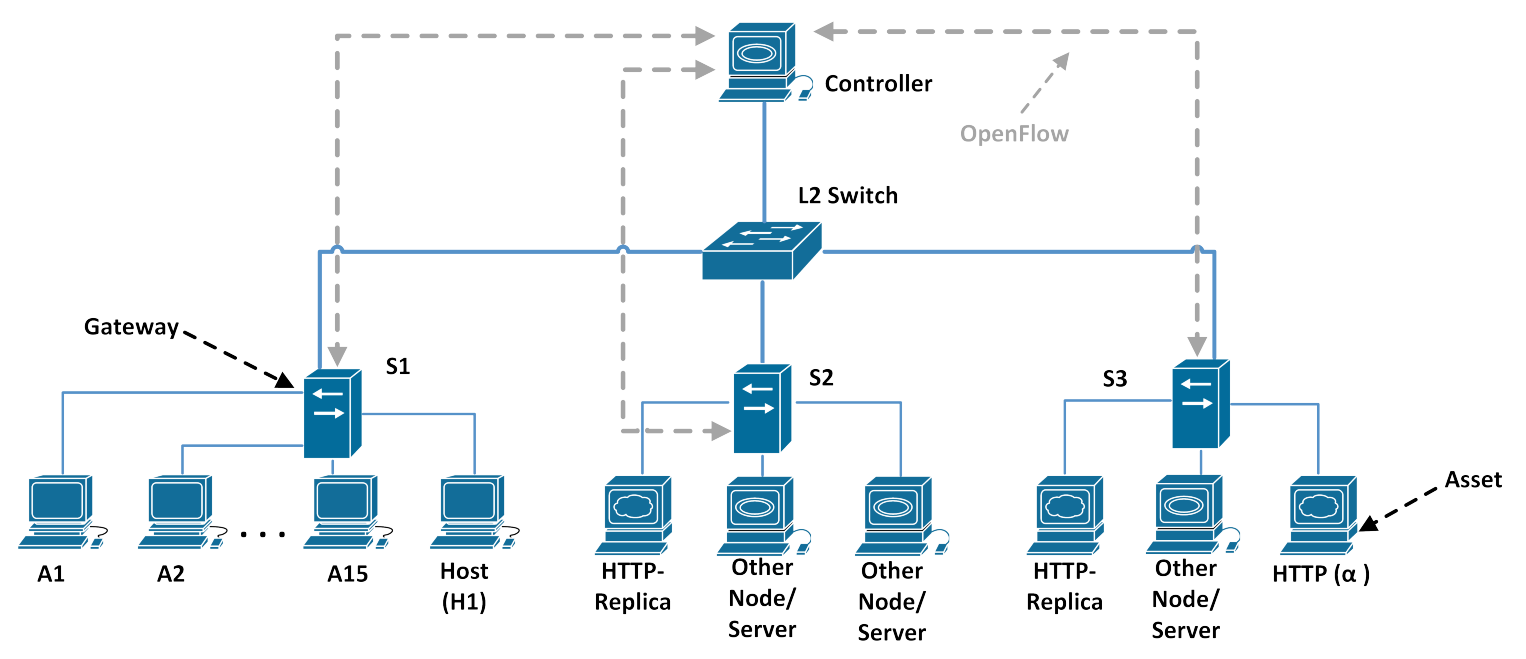

Figure 4.2: Logical Topology

\subsection{Experimental Setup and Implementation}

For the experimental setup, we have four physical machines with multiple LAN cards and one layer two switch. Each machine has $8 \mathrm{~GB}$ RAM, Intel quad-core $2.4 \mathrm{GHz}$ processor, and Ubuntu server 14.04 LTS. Three machines are acting as an Open vSwitch and end hosts. The last machine is running a Python based controller POX (version dart) [32].

Fig. 4.2 shows the logical experimental topology. In this figure, S1 is acting as a gateway switch. Whereas the specific resource (HTTP server $\alpha$ ) is attached to switch S3. $\alpha$ and its replicas are running an instance of LigHttpd [33] web server. Attacker hosts A1 to A15, and a legitimate host $\mathrm{H} 1$ is connected to gateway node $\mathrm{S} 1$, and Linux namespace containers are used to isolate hosts from each other. Three nodes ( $\alpha$ and two HTTP-Replica) are used to spread the traffic adaptively, and the POX controller's built-in method is used to collect statistics from the gateway switch every one second.

Adaptive Bubble Burst is implemented in Python as an additional module for the 
POX SDN controller. We assumed a scenario where a legitimate user needs to access or download a file from the web server, but the web server is unavailable due to a DDoS attack. In our experiment, $\mathrm{H} 1$ wants to download an image file sample.jpg from the web server, but DDoS attacker nodes A1 to A15 are also persistently requesting the same file from the server, and $\alpha$ does not have enough handling capacity to deal with all the requests. The legitimate host and attackers use a simple Python-based script to request a file from the web server. Table 4.1 summarizes the experimental parameters.

Table 4.1: ABB experiment parameters

\begin{tabular}{r|l}
\hline Parameter & Value \\
\hline$\lambda_{s}$ & 3200 packets/sec \\
$N$ & 3 \\
$T_{\text {Max }}$ & $12 \mathrm{Sec}$ \\
DDoS sources & 15 \\
H1 request rate & $1 / \mathrm{sec}$ \\
\# of Legitimate hosts & 1 \\
DDoS node request rate & $12 / \mathrm{sec}$ \\
H1 request timeout $R_{\text {timeout }}$ & 1 or $10 \mathrm{sec}$ \\
\hline
\end{tabular}

$\lambda_{s}$ is based on the number of requests server can handle multiplied with average packets per second that each request will generate. 


\subsection{Results}

We note that three servers are adequate in this setup because this setup can adequately show the effectiveness of our proposed solution ${ }^{1}$. Other setups might need more servers. In some cases, there will be incomplete requests, no matter the number of servers. We had three different scenarios, each containing multiple experiments. In each scenario, we had different $\lambda_{c}$ (includes DDoS traffic). H1 sends a HTTP request to $\alpha$ at a constant rate and waits for the response for a certain amount of time. Whereas a variable number of DDoS nodes send HTTP requests to $\alpha$ as fast as possible with very short response timeout. As mentioned in table 4.1, $\alpha$ can handle only 80 requests per second (3200 packets per second) therefore, if $\lambda_{c}$ is greater than $\lambda_{1}, \alpha$ becomes unavailable. Each experiment is repeated five times for better overall accuracy.

\subsubsection{Scenario 1}

The objective of the first scenario is to see the successful request completion for the H1 during a DDoS attack. H1 will send a total of 500 requests and have $R_{\text {timeout }} 1$ second. We will be performing a constant rate DDoS attack using 14 hosts. We have 3 cases in the current experiment i.e., DoSNoABB, ABB with 2 servers, and ABB with 3 servers. As shown in fig. 4.3, when there is a DDoS and ABB is disabled, only $4 \%$ of legitimate requests are completed with a very high response time (not shown here). On the other hand, when the experiment is repeated, and ABB is enabled with two servers, the request completion rate goes from $4 \%$ to $81 \%$ because now ABB is adaptively spreading the traffic across two servers. However, there are still

\footnotetext{
${ }^{1}$ Two servers are not enough because they will not be able to show how ABB can dynamically balance the traffic. Another problem with less than three servers is that they will not be able to deal with all the DDoS traffic
} 
some incompleted requests due to excessive traffic. In the last case where ABB is working with three servers, there are almost no timed-out requests because ABB is dynamically spreading traffic to three servers.

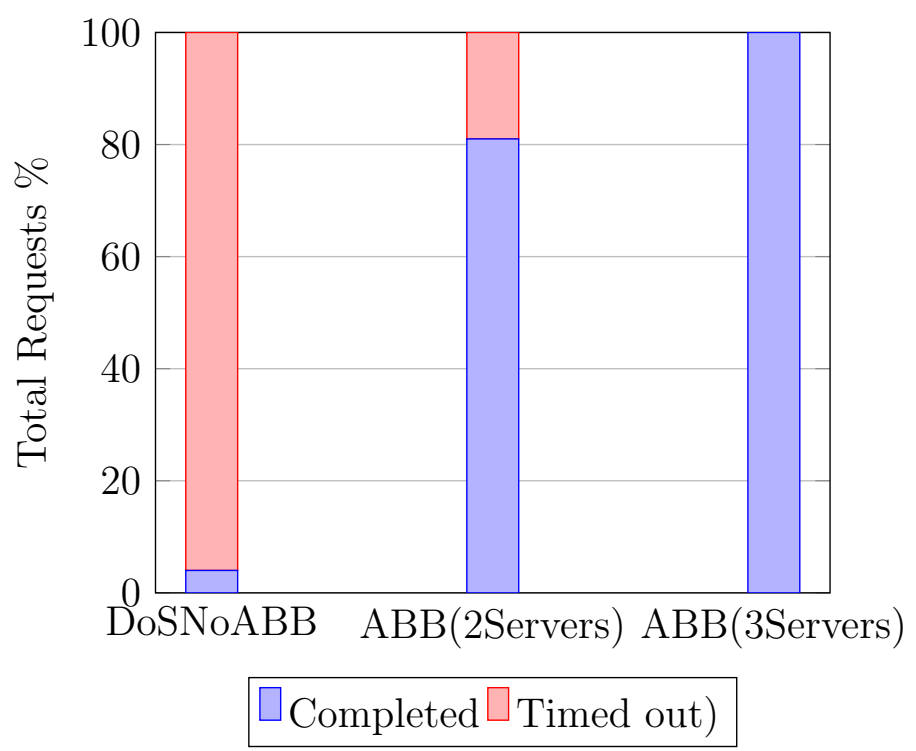

Figure 4.3: Ratio of completed and timed out requests

\subsubsection{Scenario 2}

In the second scenario, we measured the response time for four different cases (each case is a different experiment). In the first case, when there is NoDoS. Fig. $4.4\left(\log _{10}\right.$ scale) shows that the average response time is approximately 40 milliseconds. This time includes the controller's delay in finding the path and installing flows in each of the required switches. NoDoS time gives us the baseline for comparison with ABB. In order to get response time for most requests, we relaxed $R_{\text {timeout }}$ to 10 seconds. There are some incomplete requests for the $\mathrm{DDoS}$ without $\mathrm{ABB}$ and $\mathrm{ABB}$ with 2 servers because some requests are timed-out. These requests are not included in the results. Fig. 4.4 shows that ABB performs much better than the case where no protection 
against the DDoS is enabled (DoSNoABB). The difference is on the order of hundreds of milliseconds. When ABB is fully operational with three servers, its response time is almost similar to the case when there is NoDoS attack.

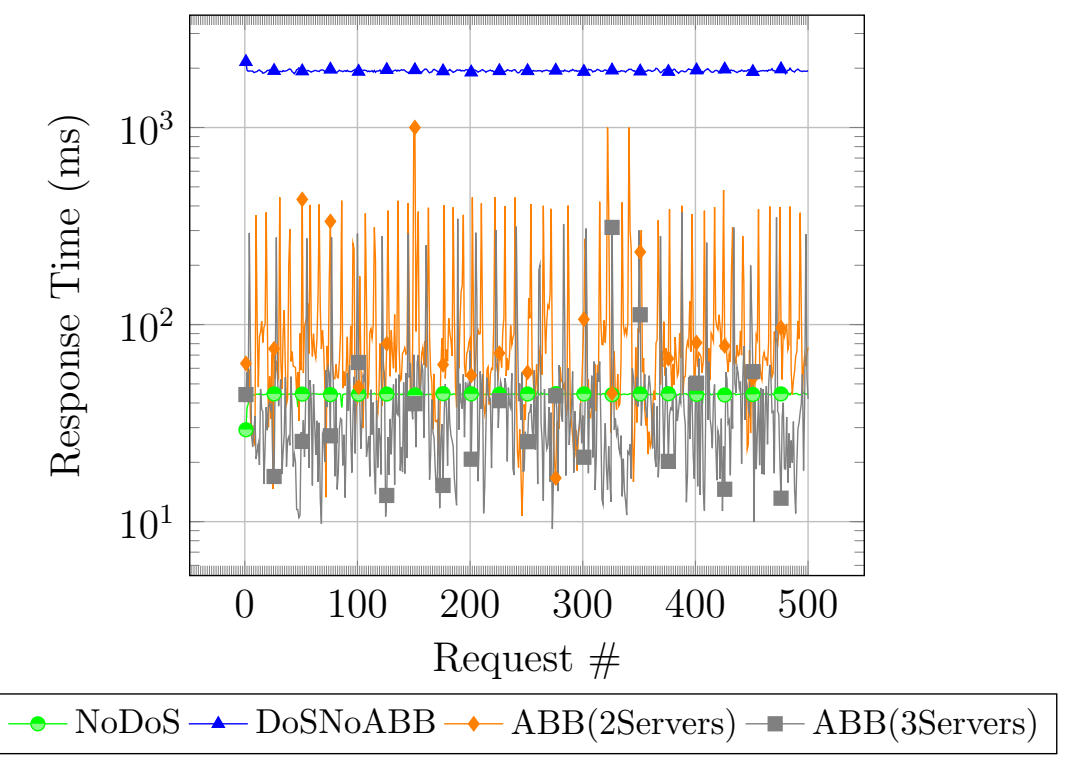

Figure 4.4: Response time comparison for constant rate DDoS

\subsubsection{Scenario 3}

Fig. 4.5 shows adaptiveness of ABB. In this scenario, we increase the DDoS attack at a steady rate. $\lambda_{c}$ starts from 61 requests per second. Every 50 requests, we add another DDoS attack node. It can be seen that although $\lambda_{c}$ is increasing at a steady rate (orange line), but the response time of legitimate host $\mathrm{H} 1$ is consistently between 20 to 70 milliseconds. At approximately request 105, another server is added to handle the attack traffic, bringing response time with a reasonable interval. On the other hand, once $\lambda_{c}$ reaches requests 160 per second, $\mathrm{ABB}$ recalibrates the number of servers to balance the traffic, therefore further reducing the response time for H1. As $\lambda_{c}$ decreases, $\mathrm{ABB}$ re-adjusts $n$ to accommodate the change in traffic. When $\mathrm{ABB}$ 
changes the number of servers, it does not need to do this sequentially. The new value for the number of servers will depend on $\lambda_{c}$.

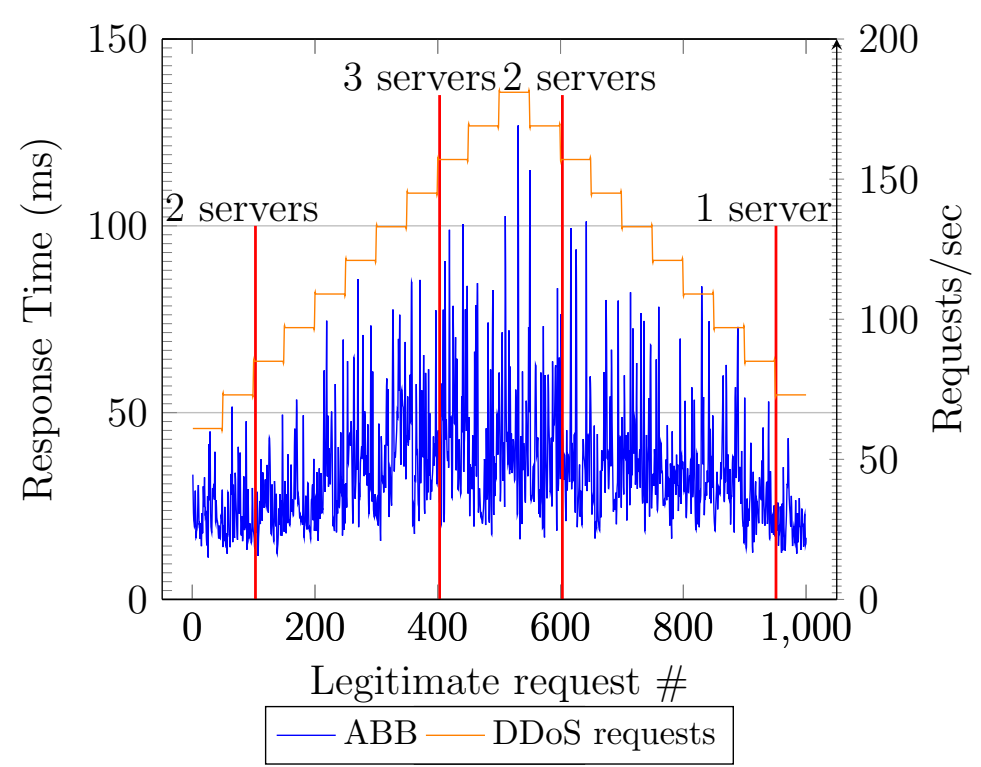

Figure 4.5: Load vs. Response time vs. Number of server

\subsection{Summary}

ABB comes at the cost of per-packet processing overhead. We used the same DDoS attack pattern of scenario 3 and calculated average packet processing time for the controller with and without ABB. Data is collected for approximately 1.4 Million packets for both cases. In the first cases, when ABB is disabled, the average packet processing time is $542 \mu \mathrm{s}$. For the second case, when ABB is enabled, it takes $776 \mu$ s to process a packet. This increase in packet processing time is due to in-part statistics collection from switches, network delay, and response time from switches. Although these values are specific to the controller's processing power and switch CPU, the overall relationship between these numbers should remain similar.

The results show that $\mathrm{ABB}$ enhances the availability of a specific resource during 
DDoS attacks. ABB mitigates DDoS attacks by adaptively spreading the incoming traffic across replicas of a specific resource in the network. ABB spread the DDoS traffic and successfully enhanced the response time to end-users. Although it has a high per-packet processing cost, the benefits of using ABB against DDoS outweigh the overhead. Packet processing overhead could be further reduced by using faster methods for collecting statistics. 


\title{
Chapter 5
}

\section{Optimal Slice Allocation in 5G Core}

\section{Networks}

\author{
66 Theres no silver bullet solution with cybersecurity, a layered \\ defense is the only viable defense. 99
}

James Scott

5G network is envisioned to be an agile and elastic network. Network slicing has emerged as a key to realizing this vision. In $5 \mathrm{G}$ networks, an end-to-end network slice is a complete logical network that includes Radio Access Network (RAN) and Core Network (CN), and it has capabilities to provide different telecommunication services [34]. An end-to-end slice is created by pairing the RAN and core network slice, but the relationship between both slices could be 1-to-1 or 1-to-M. For instance, one RAN slice could be connected to multiple core slices and vice versa $[15,35]$. Fig. 4.2 shows an example of the relationship between core and RAN slices as well as $5 \mathrm{G}$ network slicing use cases. In Fig. 4.2, two different use cases for $5 \mathrm{G}$ network slicing are shown, i.e., IoT and Remote Health Services.

The first issue we consider in slice allocation is intra-slice isolation (physical isolation between Virtual Network Functions (VNF) of a slice). The slice might require 


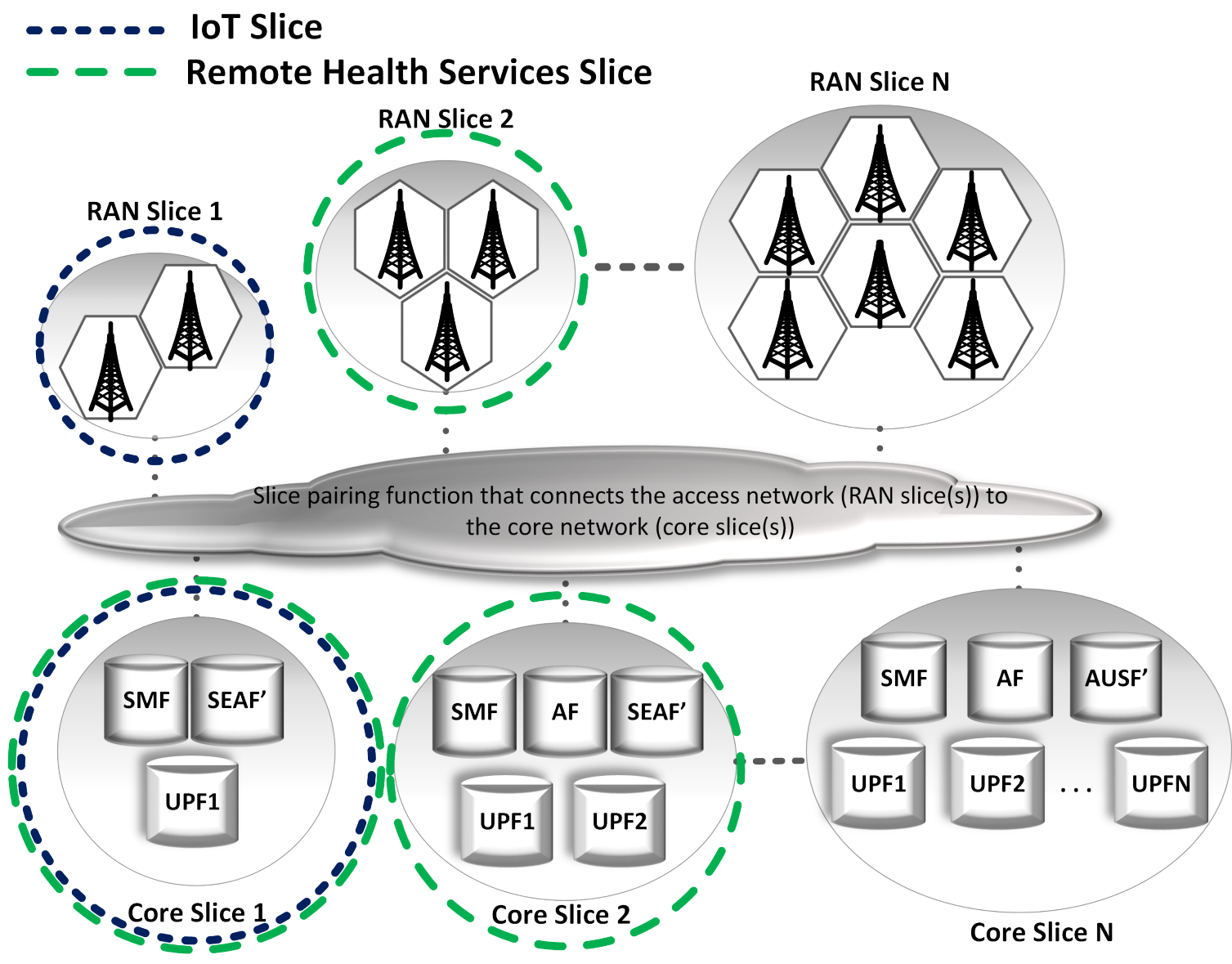

Figure 5.1: An example of Network Slicing - A RAN slice can be connected with one or more core slice and vice versa. The slice pairing function is used to connect the RAN-core slice pair.

this for more availability because if the entire slice is hosted on the same server, and if the server is compromised or becomes unavailable, the entire slice would also be affected (compromised/unavailable). However, if there is some level of intra-slice isolation, the slice operator might recover from partial compromise/unavailability of the network slice. We note that our aim does not include inter-slice isolation where other aspects need to be taken into consideration, including but not limited to physical isolation, hardware-based isolation, virtual machine-based isolation [36]. However, our focus is on providing on-demand physical isolation between different VNFs of a slice for added availability and security. 
The second issue we consider is the end-to-end delay. 5G networks have strict requirements for the end-to-end delay. To support real-time applications (e.g., health services, autonomous driving, etc.) $5 \mathrm{G}$ network needs to guarantee end-to-end delay for a specific application across the network (only considering an end-to-end delay for a core network slice).

This chapter addresses the question of optimal slice allocation in $5 \mathrm{G}$ core networks (virtual Evolved Core (vEPC)). We do this by adopting and extending VNF placement in the LTE core network presented by D. Dietrich et al. [37]. Our contributions are to (1) guarantee end-to-end delay, (2) provide intra-slice isolation for slice allocation, and (3) find a minimum delay path between the slice components. We aim to provide an optimal solution for allocating a core network slice in 5G networks. The formulation we use for the optimization model is Mixed-Linear Integer Programming (MILP). We take into consideration some of the core requirements for allocating a 5G network slice. We consider the physical isolation requirement between different components of a slice for a variable degree of availability. The optimization model also ensures the end-to-end delay required by a core network slice. A 5G network slice creation would be dynamic, and a slice could have a variable number of components that require on-demand service chaining (network slices might have a different combination of VNFs). For instance, a slice could have several components, e.g., Authentication Server Function (AUSF), Security Anchor Function (SEAF), Session Management Function (SMF), Application Function (AF) and several User Plane Functions (UPFs) with on-demand service chaining between them. We are aware that several other requirements and properties need to be addressed before a complete endto-end 5G slice can be instantiated, but those requirements are out-of-the-scope of this work. 


\subsection{MILP Formulation}

In this section, we will explain the optimization model we used in this work. We are adopting and extending the work presented by D. Dietrich et al. in [37]. The focus of their work was on the LTE cellular core and placement of network functions in an optimal manner while load balancing the resources. They transformed the optimization problem into Linear Programming (LP) problem by relaxing some MILP constraints to reduce time complexity. We adopt their model to achieve optimal slice allocation. Our objective is to allocate $5 \mathrm{G}$ core network slice VNFs optimally to provide intra-slice isolation for added availability. We use CPU utilization in our objective function because it is usually a relatively more scarce resource compared to RAM and Hard Disk Drive (HDD). Whereas, end-to-end delay is one of the core requirements for the $5 \mathrm{G}$ network.

In the following MILP formulation, we use the same network model, request model, and variables (Table 1 [37]) as in [37]. We use an undirected graph $G_{S}=\left(V_{S}, E_{S}\right)$ to represent the physical 5G core network topology. The $V_{S}$ are all the nodes in the network, and $E_{S}$ are all the physical links between the nodes. Whenever a flow is assigned to an edge $(u, v) \in E_{S}$, it incurs a delay denoted by $d_{u v} . r_{u}$ represents the remaining CPU capacity of a physical server, and $r_{u v}$ is the available bandwidth of a physical link. A directed graph denotes a slice request $G_{F}=\left(V_{F}, E_{F}\right)$, where $V_{F}$ and $E_{F}$ represent VNFs and requested service chains, respectively. Each edge in the graph is represented by $(i, j) \in E_{F}$. Each slice request is associated with a computing demand $\left(g^{i}\right)$, bandwidth requirement $\left(g^{i j}\right)$, end-to-end delay $\left(d_{E 2 E}\right)$ and intra-slice isolation (availability) required between the VNFs $\left(K_{r e l}\right)$. The description 
Table 5.1: Variable description of intra-slice isolation optimization model

\begin{tabular}{r|l}
\hline Parameter & Description \\
\hline$V_{S}$ & Set of physical Nodes \\
$V_{F}$ & Requested set of slice VNFs \\
$E_{S}$ & Physical links between nodes \\
$E_{F}$ & Requested virtual service chains of a slice \\
$g^{i}$ & CPU demand of VNF i in $\mathrm{GHz}$ \\
$g^{i j}$ & Bandwidth demand of the edge $(\mathrm{i}, \mathrm{j})$ in $\mathrm{Mbps}$ \\
$r_{u}$ & Remaining capacity of the server $\mathrm{u}$ in $\mathrm{GHz}$ \\
$r_{u, m a x}$ & Maximum capacity of the server $\mathrm{u}$ in $\mathrm{GHz}$ \\
$r_{u v}$ & Remaining capacity of the link $(\mathrm{u}, \mathrm{v})$ in $\mathrm{Mbps}$ \\
$r_{u v, m a x}$ & Maximum capacity of the link $(\mathrm{u}, \mathrm{v})$ in $\mathrm{Mbps}$ \\
$d_{u v}$ & Delay of the link (u,v) in ms \\
$\gamma_{u}^{i}$ & indicates, if VNF i can be allocated to server $\mathrm{u} . \gamma_{u}^{i} \in 0,1$ \\
$x_{u}^{i}$ & Assignment of VNF i to server u \\
$f_{u v}^{i j}$ & Amount of bandwidth assigned to the link $(\mathrm{u}, \mathrm{v})$ for the edge $(\mathrm{i}, \mathrm{j})$ in $\mathrm{Mbps}$ \\
$\alpha^{i}$ & Processing delay of a VNF \\
\hline &
\end{tabular}

of all variable is provided in table 5.1. We use the following objective function.

Minimize

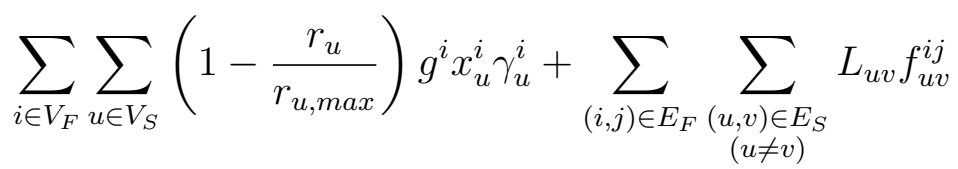

subject to:

$$
\sum_{u \in V_{S}} x_{u}^{i}=1 \quad \forall i \in V_{F}
$$


CHAPTER 5. SLICE ISOLATION USING OPTIMAL RESOURCE ALLOCATION 47

$\sum_{\substack{\left.(u, v) \in V_{S} \\ u \neq v\right)}}\left(f_{u v}^{i j}-f_{v u}^{i j}\right)=g^{i j}\left(x_{u}^{i}-x_{u}^{j}\right) \quad i \neq j, \forall(i, j) \in E_{F}, \forall u \in V_{S}$

$\sum_{i \in V_{F}} g^{i} x_{u}^{i} \leq r_{u} \quad \forall u \in V_{S}$

$\sum_{(i, j) \in E_{F}} f_{u v}^{i j} \leq r_{u v} \quad \forall(u, v) \in E_{S}$

$\left(\sum_{(i, j) \in E_{F}} \sum_{\substack{(u, v) \in E_{S} \\ u \neq v}}\left(\frac{f_{u v}^{i j}}{g^{i j}} d_{u v}\right)+\sum_{i \in V_{F}} \alpha^{i}\right) \leq d_{E 2 E}$

$\sum_{i \in V_{F}} g^{i} \leq \sum_{u \in V_{S}} r_{u}$

$\sum_{(i, j) \in E_{F}} g^{i j} \leq \sum_{(u, v) \in E_{S}} r_{u v}$

$\sum_{i \in V_{F}} x_{u}^{i} \leq K_{r e l} \quad \forall u \in V_{S}, K_{r e l}=1,2,3 \ldots$ 
The objective function (5.1) will assign the incoming slice requests to the least utilized server and find a path with minimum delay. The first term is identical to the objective function in [37], while the second one differs in the way we select paths between VNFs. The first term of the objective function assigns computing demands to the least utilized physical servers. The parameter $\gamma_{u}^{i}$ is used to indicate if VNF $i$ can be allocated to server $u^{1}$. The second term takes into consideration the physical link delay $\left(L_{u v}\right)$. Each time when a virtual link $(i, j) \in E_{F}$ is assigned to a physical link $(u, v) \in E_{s}$, it increases $L_{u v} . \quad L_{u v}$ is a function of link utilization, and it is calculated using eq. (5.10), where $L_{u v, \text { init }}$ is the initial delay assigned to the link $(u, v) \in E_{s}$. Minimizing both terms will result in the assignment of a network slice to the least utilized servers, and it will find a path with the least delay between the slice components.

$$
L_{u v}=\left(1-\frac{r_{u v}}{r_{u v, \max }}\right) 2.5 m s+L_{u v, \text { init }} \quad \forall(u, v) \in E_{S}
$$

The objective function is subjected to several MILP constraints that we will explain next. In our work, in addition to the constraints listed here, we use constraints (5.2), (5.3) , (5.4), and (5.5) from [37]. If the slice has requested that each VNF need to be assigned to different physical servers, constraint (5.9) will provide the desired degree of availability (intra-slice isolation) for the slice $\left(K_{r e l}\right)$. For instance, if a slice requests an intra-slice isolation level of five (i.e., $K_{r e l}=5$ ), then constraint (5.9) will ensure that number of VNFs hosted on server $u$ are five or less. The end-to-end delay for the $5 \mathrm{G}$ network is an important requirement. Constraint (5.6) enforces the

\footnotetext{
${ }^{1}$ The dataset $V_{S}$ contains all the servers, routers, and switches
} 
end-to-end delay requirement for the core network slice ${ }^{2}$. It includes the delay incurred along the entire path and the processing delay of each $\operatorname{VNF}\left(\alpha^{i}\right)$. The term $\left(\frac{f_{u v}^{i j}}{g^{i j}}\right)^{3}$ will only be equal to one when a virtual link $(i, j)$ is assigned to a physical link $(u, v)$. Therefore, only physical links that have reserved the bandwidth for a slice will be included in the end-to-end delay. Since the partial or incomplete assignment of the slice components serves no purpose, constraints (5.7) and (5.8) ensures that the remaining computing and bandwidth capacity of the entire data center is enough to accommodate the slice creation request. $x_{u}^{i} \in 0,1$ and $f_{u v}^{i j} \geq 0$ are binary and real decision variables, respectively.

\subsection{Results and Discussion}

Table 5.2: Intra-slice isolation simulation parameters

\begin{tabular}{r|l}
\hline Parameter & Value \\
\hline CPU capacity/server $\left(r_{u, \text { max }}\right)$ & $12.0 \mathrm{GHz}$ \\
Total Servers $\left(V_{S}\right)$ & 200 \\
Total Slice Requests & 200 \\
$K_{r e l}$ & $1-10$ \\
VNF/slice $\left(V_{F}\right)$ & 10 \\
Bandwidth request/slice $\left(g^{i j}\right)$ & $30-70 \mathrm{Mbps}$ \\
VNF CPU request/slice $\left(g^{i}\right)$ & $0.5-2.0 \mathrm{GHz}$ \\
$\alpha^{i}$ & $0.3-2.0 \mathrm{~ms}$ \\
\hline
\end{tabular}

To test the optimization model, we used MATLAB to simulate 5G core network

\footnotetext{
${ }^{2}$ We note that meeting end-to-end delay requirements would need traffic engineering, which is outside the scope of this work.

${ }^{3} f_{u v}^{i j}$ is the allocated bandwidth and $g^{i j}$ is the request bandwidth
} 
and slice requests. AMPL is used to model optimization algorithm and CPLEX 12.8.0.0 is used as MILP solver. The optimization algorithm is evaluated on Intel Core i7 $3.2 \mathrm{GHz}$ with $32 \mathrm{~GB}$ RAM.

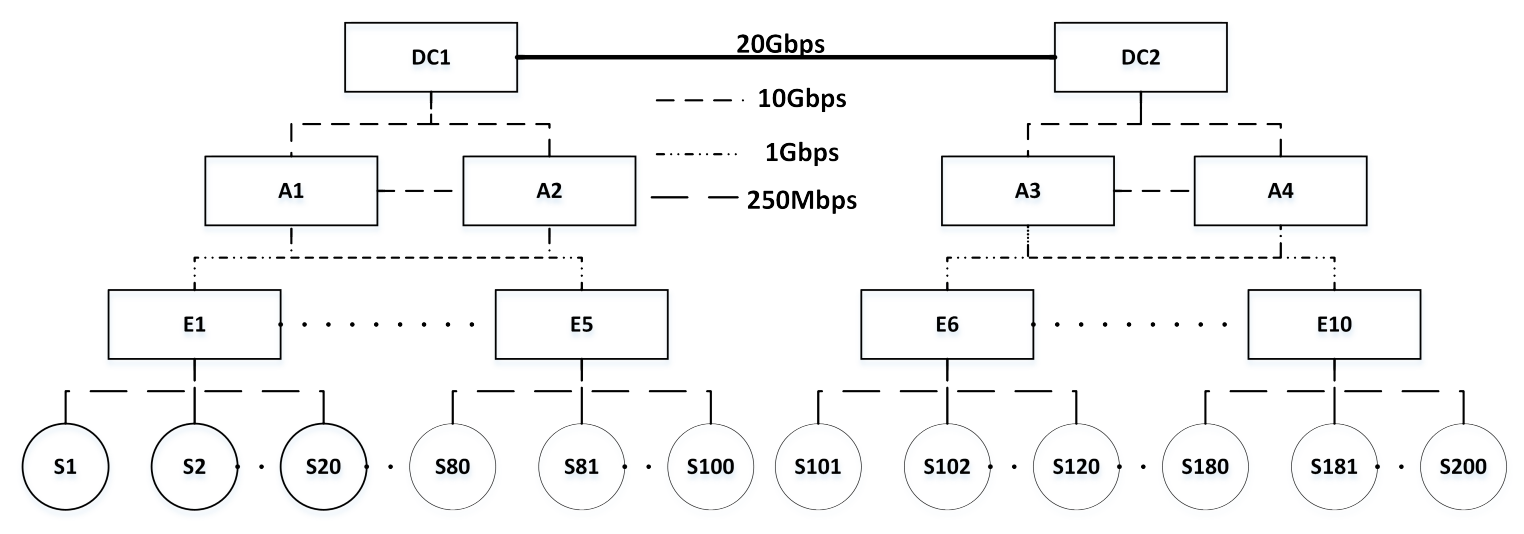

Figure 5.2: Simulation topology. S1-S200, E1-E10, A1-A4, and DC1-DC2 represent physical servers, Edge, Aggregation and Datacenter switches, respectively

We simulate 200 physical servers that can host different types of VNFs. Other parameters used for the evaluation are listed in Table 5.2. In our simulations, we vary the level of intra-slice isolation using the $K_{\text {rel }}$ parameter. This parameter provides the upper limit for how many VNFs can be placed on one physical server. The model guarantees the requested computing resources, bandwidth resources, and end-to-end delay for a slice in the current network state. After allocating each slice, we update the remaining computing and bandwidth resources. The flow link delay $L_{u v}$ can be dynamic. For instance, when the network is congested, this parameter can be updated to reflect the network's current state, but we did not consider this case.

In our simulations, we used two configurations for link bandwidth (Servers $\leftrightarrow$ EdgeSwitches). In the first configuration, as shown in Fig. 5.2, the link bandwidth between servers and edge switches is set to $250 \mathrm{Mbps}$. In this case, the overall system performance is limited by the available CPU capacity (CPU bound). Therefore, our simulated slice requests, the physical servers' CPU capacity, become 


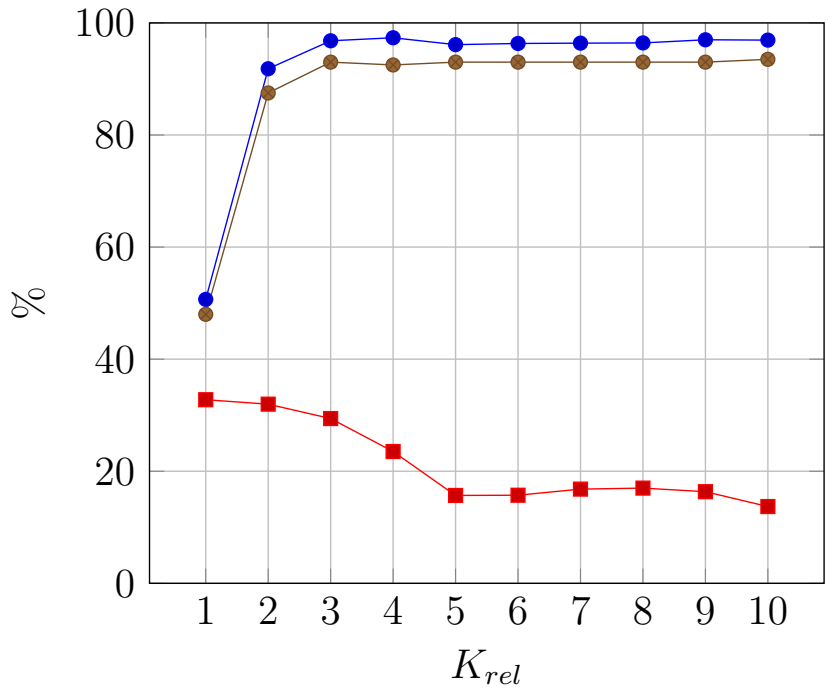

- CPU utilization - Bandwidth utilization - Requests accepted

Figure 5.3: CPU utilization, bandwidth utilization and requests accepted for varying levels of $K_{r e l}$ (CPU Bound)

the limiting factor when allocation slices rather than the link bandwidth. In the second configuration, the link bandwidth (Servers $\leftrightarrow$ EdgeSwitches) is set to $100 \mathrm{Mbps}$ (Bandwidth bound). In this case, the overall system performance is limited by the available link bandwidth between servers and Edge switches. Please note that in all the presented results, the simulation setup was "CPU bound" unless otherwise stated.

\subsubsection{Intra-slice isolation}

In the first part of the simulation, we fix the end-to-end delay $\left(d_{E 2 E}\right)$ to a relatively high value $(500 \mathrm{~ms})$ to minimize its effect on the results and vary the levels of intraslice isolation $\left(K_{r e l}\right)$. Fig. 5.3 shows the overall average system utilization for CPU and bandwidth resources and accepted requests for different levels of intra-slice isolation. The system is CPU bound so that overall system bandwidth is higher than the total 
CHAPTER 5. SLICE ISOLATION USING OPTIMAL RESOURCE ALLOCATION 52

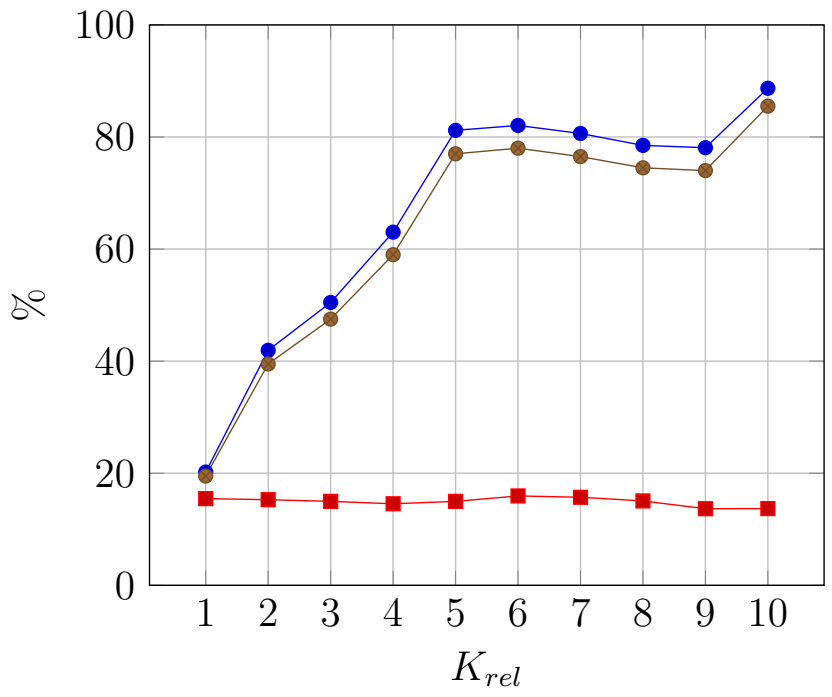

- CPU utilization - Bandwidth utilization $\multimap$ Requests accepted

Figure 5.4: CPU utilization, bandwidth utilization and requests accepted for varying levels of $K_{r e l}$ (Bandwidth Bound)

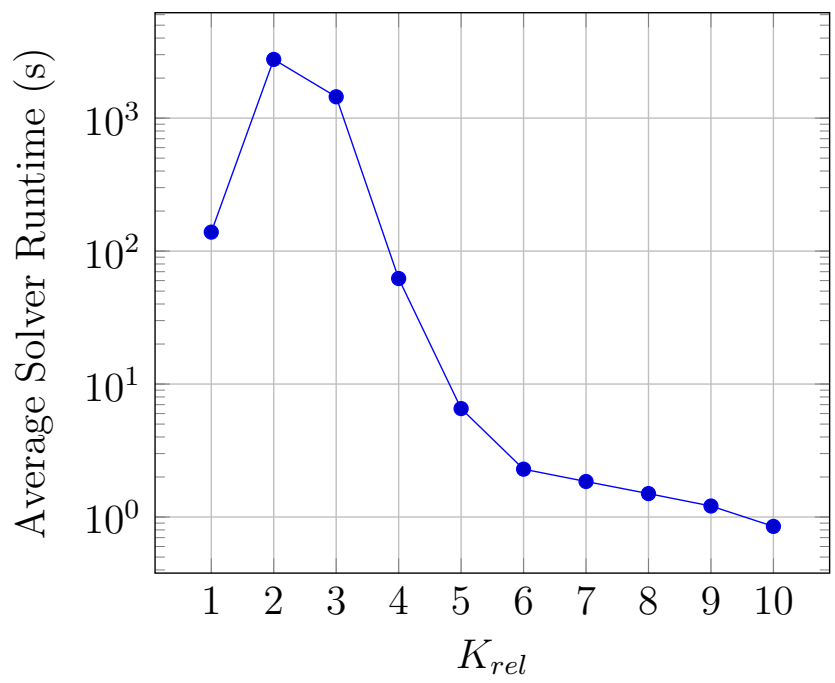

Figure 5.5: Average solver runtime (seconds) for varying levels of $K_{\text {rel }}$

requested bandwidth. Hence we see relatively low bandwidth utilization. When slices request intra-slice isolation where $K_{r e l}<4$, the bandwidth utilization is higher because all VNFs would have to utilize the physical links to communicate with each other. Whereas, when we relax the intra-slice isolation requirement, we get lower 


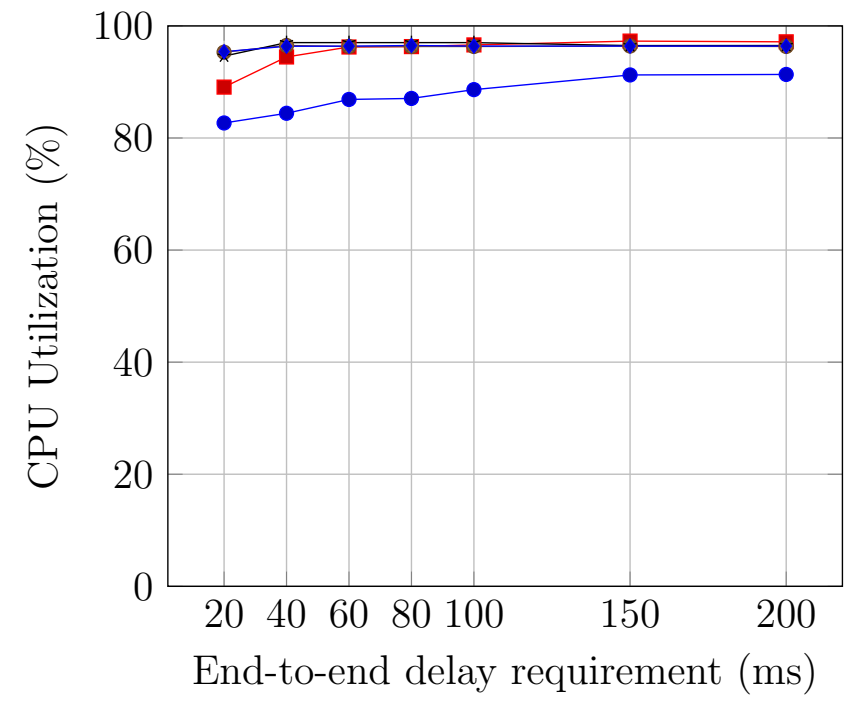

$\bullet K_{r e l}=2 \multimap K_{r e l}=4 \multimap K_{r e l}=6 \multimap K_{r e l}=8 \multimap-K_{r e l}=10$

Figure 5.6: CPU utilization for different end-to-end delay requirements

network utilization (i.e., $K_{r e l} \geq 4$ ). The reason is that we can allocate more VNFs on the same physical server, and the communication between the VNFs does not involve a physical communication link; we see lower network activity in this case. However, there is a marginal difference in CPU utilization and requests accepted for variable levels of $K_{r e l} \geq 2$.

We also simulated another topology where the system was bandwidth bound. Fig. 5.4 shows the overall system utilization for CPU, bandwidth, and requests accepted. The performance of the system is worse compared to when the system is CPU bound (Fig. 5.3).

Fig. 5.5 provides some interesting results for the average solver runtime. Obviously, with stricter requirements for intra-slice isolation are, more time is required to find an optimal solution for the allocation of slice components and to find an optimal path with the least delay. A factor that impacts these values is that when the requirement for intra-isolation is flexible, the optimization algorithm can place more 
components on the same physical system, and it would eliminate the need to find optimal paths between these components. We can see this behavior when $K_{r e l}>4$ in Fig. 5.5. However, as we can see, when a slice requests that no more than two or three VNFs can be placed on a single physical server, there is a significant variation in solver runtime. We ran these simulations multiple times and using multiple parameter values, and each time we obtain almost identical results. We have not been able to identify the reason behind the anomalous behavior for $K_{r e l}=2$ and $K_{r e l}=3$.

\subsubsection{End-to-end delay}

In the second part of the simulation, we use different end-to-end delay requirements. Please note that we ran simulations for $K_{r e l}=1$ to $K_{r e l}=10$, but results are only shown for a few values of $K_{\text {rel }}$ to present more readable graphs. The end-to-end delay parameter has a noticeable effect on CPU utilization because setting $K_{r e l} \leq 2$ reduces the number of available solutions, as shown in Fig. 5.6. However, this effect becomes minimum when $d_{E 2 E} \geq 150$. We note that the CPU utilization shows the same behavior as the request acceptance rate (not shown here).

Fig. 5.7 shows that different end-to-end delay requirements have minimum impact on overall bandwidth utilization for all levels of $K_{r e l}$. Fig. 5.8 shows the average solver runtime for different end-to-end delay requirements. We can see consistent behavior for all levels of intra-slice isolation. 
CHAPTER 5. SLICE ISOLATION USING OPTIMAL RESOURCE ALLOCATION 55

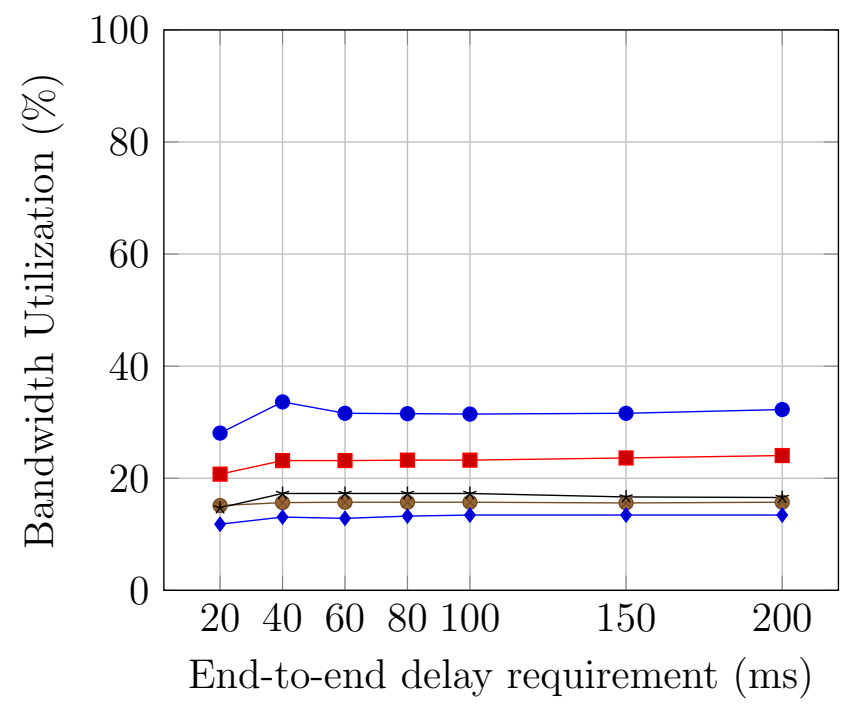

$\multimap K_{r e l}=2 \multimap-K_{r e l}=4 \multimap K_{r e l}=6 \multimap K_{r e l}=8 \multimap K_{r e l}=10$

Figure 5.7: Bandwidth utilization for different end-to-end delay requirements

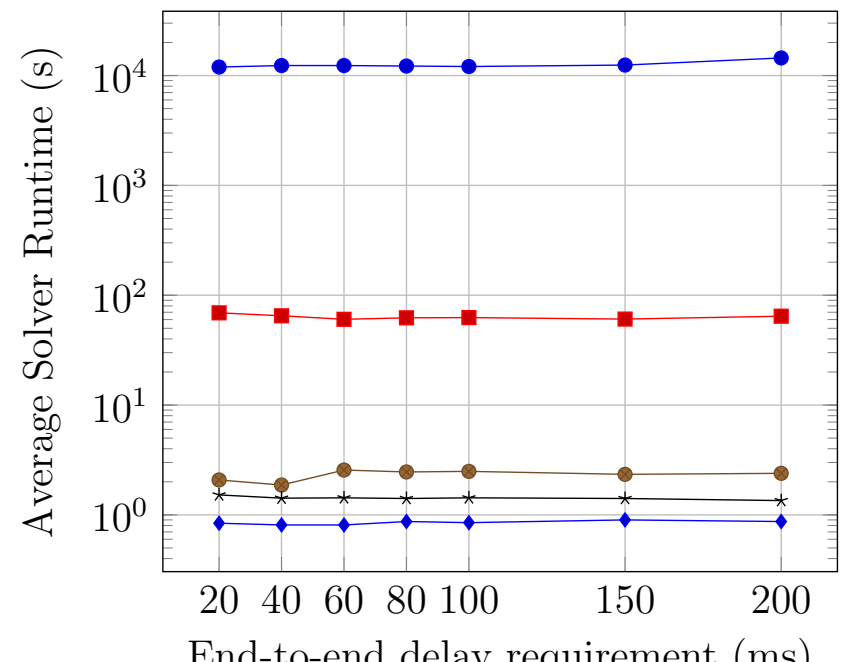

$\bullet K_{r e l}=2 \multimap K_{r e l}=4 \multimap K_{r e l}=6 \multimap K_{r e l}=8 \multimap K_{r e l}=10$

Figure 5.8: Average solver runtime (seconds) for different end-to-end delay requirements 


\subsection{Summary}

In this chapter, we addressed the optimal allocation of $5 \mathrm{G}$ core network slices. The optimization model provides intra-slice isolation as well as ensures that the end-toend delay meets the minimum requirement. We evaluated the optimization model by simulating a virtualized mobile core. Our evaluation shows that when there is little or no restriction on the intra-slice isolation $\left(K_{r e l}>2\right)$, CPU utilization is increased, and the demand for bandwidth is reduced due to the reduction between inter-machine communications. On the other hand, stricter intra-slice isolation $\left(K_{r e l} \leq 2\right)$ requires more bandwidth and leads to relatively lower CPU utilization. 


\title{
Chapter 6
}

\section{Towards Secure Slicing: Using Slice Isolation to Mitigate DDoS Attacks on 5G Core Network Slices}

\author{
66 If security were all that mattered, computers would never be \\ turned on, let alone hooked into a network with literally \\ millions of potential intruders. 99
}

Dan Farmer

A shift in the mobile network paradigm also mandates changes in the security architecture with new security challenges that do not exist in the past or present mobile networks [3]. In the current mobile network, if an attacker launches a flooding Distributed Denial of Service (DDoS) attack against a mobile service, only that service will be affected, and there is a very low risk of host resource starvation-based attacks in current mobile networks (i.e., usually mobile network infrastructure is not virtualized). However, in a 5G network, a DDoS against one slice might affect other services because they could be tenants of the same shared virtualized infrastructure. An important requirement for $5 \mathrm{G}$ network slicing is isolation [3]. Network slice isolation requires host resource isolation and network communication isolation that could 
be achieved using various techniques suggested in [36]. Each suggested technique has its advantages and disadvantages. However, separating hardware resources provides strong isolation.

In this chapter, we try to mitigate the Distributed Denial-of-Service (DDoS) attacks using slice isolation. We utilize a mathematical model to solve a security problem (DDoS). In our proposed solution, we use slice isolation as security constraints for the optimization model and proactively mitigate DDoS attacks. Our contributions are: (1) inter-slice isolation (complete separation of host hardware resources between slices), (2) intra-slice isolation (separation of host hardware resources between the slice components), (3) guaranteeing the end-to-end delay requirement for the slice, (4) optimal allocation of slices to efficiently utilize available hardware infrastructure and (5) evaluation of security constraints.

Inter-slice isolation provides mitigation against the DDoS attacks because the hardware resources are not shared between the slices, and DDoS on one slice does not impact the other slices, whereas intra-slice isolation provides better availability for the slices since the components of the slice are hosted on different hosts. Enabling intra-slice could reduce the impact of DDoS attack and increase the availability of the target slice as well as faster recovery of the service because the slice operator may not need to deploy reactive mitigation defense for all slices (e.g., migrating only affected slices).

In a 5G network, not all slices or slice components will require the same level of security. For instance, a slice's control plane function might be required to be allocated in a predefined relatively secure zone compared to the data plane functions. Therefore providing flexibility to allocate slices according to the security requirements might be necessary.

The objective of our work is to provide inter-slice and intra-slice isolation, secure, 
guarantee the end-to-end delay, make efficient use of system resources, and increase the availability of a slice against the DDoS attacks. To achieve these objectives, we use a security-focused optimization model that allocates slices and fulfills different 5G network slicing requirements. We use a combination of simulation and experimental work to evaluate our DDoS mitigation solution. We also evaluate the performance of our current optimization model. We note that we do not use slicing in 5G Radio Access Network (RAN), we only focus on core slicing.

\subsection{Related Work}

Network Slicing Security: V. Sathi et al. [39] proposed a novel protocol for securing intra-slice network communication. The proposed protocol is based on modified proxy re-encryption and bilinear pairing on an elliptic curve. It provides mutual trust between participating entities and a distributed association between slice components in a secure manner (if central orchestrator is unavailable). The authors did not consider the intra-slice isolation and end-to-end delay for a slice. An intelligent security-focused auto-scaling algorithm has been proposed by Y. Khettab et al. [40]. Authors leveraged the Network Function Virtualization (NFV) and Software-Defined Network (SDN) architecture to secure a network slice. SDN controller ONOS is used to enable security as a service in the proposed algorithm. The auto-scaling solution considers the VM's startup time and guarantees the resources at the orchestrator level by setting minimum and maximum resources for each slice. IoT is one of the major drivers behind $5 \mathrm{G}$ network development. Y. Khettab et al. did not consider secure allocation, end-to-end delay, and intra-slice isolation. An efficient and secure method is required to authenticate a massive number of low powered devices. J. Ni et al. [41] proposed a secure service-oriented authentication framework for 5G-enabled 
IoT devices $\left(E S^{3} A\right)$. The proposed framework guarantees privacy-focused secure slice access and selection as well as a secure session key exchange mechanism between IoT devices. A quantitative analysis of network slice isolation has been proposed by Z. Kotulski et al. [42]. The proposed framework is a layered structure, and each layer has its network elements and isolation level. The authors defined mathematical rules to calculate slice isolation. However, end-to-end delay, secure allocation, and intra-slice isolation were not considered.

Virtual Network Function Placement in Mobile Networks: Recently, Virtual Network Function (VNF) placement in the virtual mobile network has gained research communities' interest. The main driving force is the architecture of the $5 \mathrm{G}$ network that heavily relies on virtualization. V. Sciancalepore et al. [43] have proposed a practical implementation of network slicing. In the proposed model, authors assume that tenants can only request a single network slice in any given time slot. The proposed model is built on the multi-armed bandit problem, and the authors introduced three variations of the multi-armed bandit to solve various aspects of the network slice allocation. They simulated the optimization model using MATLAB and compared the results with a greedy algorithm. They also provided proof-of-concept implementation of the network slicing. However, the authors did not consider end-toend delay and slice isolation. D. Dietrich et al. [37] proposed a linear programming formulation for the placement of VNFs in the LTE core network. In the proposed algorithm, they provided a balance between optimality and time complexity. The proposed optimization model did not consider slice isolation, secure slice allocation, and end-to-end delay of a slice. The authors R. Ford et al. [44] proposed an optimal VNF placement for the SDN-based 5G mobile-edge cloud. Their optimization algorithm provides resilience by placing VNFs in distributed data centers. They demonstrate in the evaluation that their algorithm can reduce $75 \%$ of redundant data center capacity 
while still maintaining the same resilience. R. Ford et al. did not tackle the issue of slice isolation.

5G Testbeds: A Practical Open-Source Solution for End-to-End Network Slicing (POSENS) has been proposed by G. Aviles et al. [45]. POSENS uses open source software and hardware to create end-to-end slices. Authors used srsLTE for radio access networks and open-air interface EPC for the core network. The independence of slices and performance throughput is discussed in our work. It also supports an efficient and flexible deployment of network slices. L. Zanzi et al. [38] demonstrated a real testbed named OVerbooking NEtwork Slices (OVNES). In the testbed, authors considered three different vertical slices, i.e., Public Safety communications, enhanced Mobile BroadBand (eMBB) for voice calls, and eMBB for the Internet (best-effort). OpenEPC is used to emulate mobile core and several LTE devices to generate traffic.

\subsection{Threat Model}

In this section, we describe the threat model used in this work. Our threat model considers the $5 \mathrm{G}$ elements that are involved in network slicing.

\subsubsection{Assumptions}

Our threat model assumes that:

- Network slicing is supported by the target network

- It is possible to modify the VNF configuration of a slice

- A network slice supports the installation of user-defined functions

- The 5G core network uses virtualized network functions 
- The EPC supports migration of the slice components

- On the servers, multi-tenancy is supported by the slice operator

- It is possible to have slice-level authentication

- It is not possible to modify the hardware configurations of the baremetal systems

- The slice operators' network is functional

\subsubsection{Adversaries}

Our model considers two types of adversaries. We assume that both adversaries are not colluding, and they can determine co-residency with the target slice (if necessary).

- Adversaries with administrative control: An Adversary could be someone with administrative control of the slice. This type could be a tenant, an insider (assigned by the mobile operator to manage the slice), or an external attacker who has compromised a slice.

- External adversaries: External attacker could be a user of a slice or an external entity that could attack a slice. The adversary is authorized to communicate with one of the hosted slices.

\subsubsection{Attacks on a slice in the multi-tenant networking envi- ronment}

In this threat model, we consider two possible attacks on a slice in the multi-tenant environment. These are the two attacks we used in our experimental evaluation. 


\subsubsection{DDoS flooding attack}

Unlike LTE networks, which are usually single-tenant, multi-tenancy in 5G networks will be a typical environment. For the flooding attacks, we assume that the attacker is an external adversary (second type). The attacker launches a DDoS attack that could flood the communication link(s) of the target slice (e.g., UDP flooding, TCP sync attack, or any other type of DDoS flooding attack). DDoS flooding attacks [46], [47] are not new or limited to the mobile networks. They have existed in traditional networks, cloud networks, mobile ad-hoc networks, software-defined networks and in many other types of networks. In $5 \mathrm{G}$ networks, network slicing presents a new attack vector to perform DDoS attacks. A high-level view of the DDoS attack is shown in Fig. 6.1.

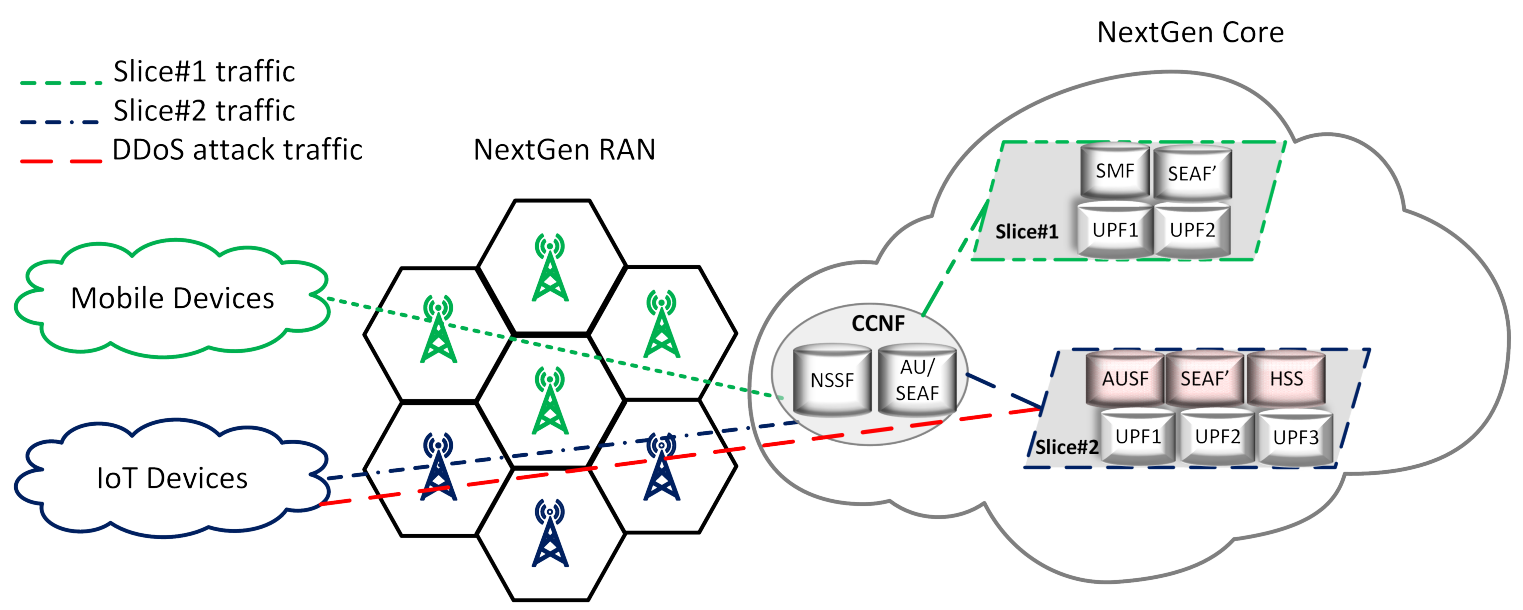

Figure 6.1: Logical view of DDoS attack on a slice

In Fig. 6.1, we have two slices providing services to two different classes of devices (i.e., Mobile devices and IoT devices). The Common Control Network Functions (CCNFs) are shared among both slices and provide slice selection and authentication functions. In this scenario, we have a large number of IoT devices attempting to authenticate with the slice\#2. This could result in a DDoS attack on slice\#2's 
components (i.e., AUSF, SEAF', and HSS). However, If both slices share the same physical link in the core network (all or some components of both slices could share the same physical link), then slice\#1 might be impacted, and it could result in an in-direct DDoS on the slice\#1.

\subsubsection{Slice-initiated attack}

There is a possibility that a slice might initiate the attack in this multi-tenant environment. A slice-initiated attack would require administrative privilege because it might require modification of $\mathrm{VNF}$ /slice configuration. We assume that the attacker has administrative control of the slice (the first type of adversary). An attacker could exhaust the resources of the VNF (e.g., 100\% CPU or RAM utilization) [47], [46]. The effect of a slice-initiated attack is limited to the slices hosted on the same host(s). If one or more components of a slice are hosted on the same physical host as the

adversarial slice $\operatorname{VNF}(\mathrm{s})$, then sustained high utilization of hardware resources could degrade other slices' performance.

\subsection{Proposed Solution}

This section describes our solution, where we provide optimal slice allocation, slice isolation, and secure allocation while fulfilling several requirements of $5 \mathrm{G}$ networks. We use intra-slice isolation to provide better availability, inter-slice isolation for providing isolation between slices, and secure allocation for added security.

In our solution, when slice allocation is requested, the slice could require intra-slice isolation for high availability. If there is a DDoS attack on the slice or a hardware failure, it could have a catastrophic effect if the entire slice is allocated on one host. An essential requirement for $5 \mathrm{G}$ network slicing is isolation [48]. The 3GPP states 
that "The 3GPP System shall have the capability to provide a level of isolation between network slices which confines a potential cyber-attack to a single network slice" [49]. However, how network isolation should be achieved is left up to the implementation of the network slicing. In our solution, if a slice requires complete isolation from other slices, the inter-slice isolation constraint provides such functionality.

\subsection{Optimization Model Formulation}

In this section, we explain the optimization model used in this work. Inter-slice isolation, guaranteeing end-to-end delay, and high availability are essential requirements in 5G network slicing [15]. Our security-focused optimization constraints fulfill different isolation requirements. Inter-slice isolation will depend on how the slicing is implemented. If multiple VNFs are assigned to the same VM from different slices, one way to achieve inter-slicing isolation would be to assign the only slice per VM (container). In this work, we consider one VNF per VM (container); therefore, it already provides some level of isolation. However, we consider complete separation of hardware inter-slice isolation (such that no components of different slices can be allocated to the same host).

\subsubsection{Network and Request Model}

We use an undirected graph $G_{p}=\left(N_{p}, L_{p}\right)$ to represent the physical $5 \mathrm{G}$ core network topology. All the nodes in the network (i.e. servers, switches, routers and other devices present in the network) are represented by $N_{p}$, and $L_{p}$ denotes all the physical links between the nodes. A slice request is denoted by a directed graph $G_{v}=\left(N_{v}, L_{v}\right)$, where $N_{v}=\left(N_{c} \cup N_{d}\right)$ contains all the slice virtual network functions, the control and data plane virtual functions are represented by $N_{c}$ and $N_{d}$, respectively and 
$L_{v}$ represents requested links. Each edge in the directed graph is represented by $(i, j) \in L_{v}$. Each slice request is associated with end-to-end delay $\left(d_{E 2 E}\right)$, intra-slice isolation $\left(K_{r e l}^{c}, K_{r e l}^{d}\right)$, inter-slice isolation $\left(\gamma^{c}, \gamma^{d}\right)$ and each VNF in a slice is associated with a computing demand $\left(R^{i}\right)$, and bandwidth (BW) requirement between VNF i and VNF $\mathrm{j}\left(R^{i j}\right)$. The description of all variable is provided in table 6.1.

\subsubsection{Objective function}

In earlier work Chap. 5 ( [50]), we used a similar objective function and general constraints. They are influenced by prior work [37] by Dietrich et al., especially the first term of the objective function and general constraints 6.3, 6.4, and 6.6.

Minimize

$$
\sum_{i \in N_{v}} \sum_{k \in N_{p}}\left(\sigma_{k}+R^{i}\right) u_{k}^{i}+\sum_{(i, j) \in L_{v}} \sum_{\substack{(e, f) \in L_{p} \\(e \neq f)}} T_{e f} y_{e f}^{i j}
$$

The objective function (6.1) is to allocate the slice to least loaded physical nodes and find a minimum delay path. The first term will assign the slice request to the least loaded servers. The second term will find the minimum delay path. Minimizing both terms will result in the assignment of a network slice to the least loaded servers, and it will find a path with the least delay between the slice components.

The objective function is subjected to several Mixed-Integer Linear Programming (MILP) general and security-related constraints that we will explain next. 
Table 6.1: Description of variables for secure slicing optimization model

\begin{tabular}{r|l}
\hline Parameter & Description \\
\hline$N_{p}$ & Set of physical Nodes \\
$L_{p}$ & Physical links between nodes \\
$\sigma_{k}$ & Current CPU load of physical node $k$ \\
$\sigma_{e f}$ & Current BW allocation of physical link between nodes $e, f$ \\
$\sigma_{k}^{m a x}$ & Maximum CPU capacity of physical node $k$ \\
$\sigma_{e f}^{m a x}$ & Maximum BW capacity of physical link between nodes $e, f$ \\
$T_{e f}$ & Physical link delay between node $e, f$ \\
$\Delta_{k}$ & Physical node $k$ processing delay \\
$\Delta^{i}$ & VNF $i$ processing delay \\
$N_{c}$ & Requested set of slice control plane functions \\
$N_{d}$ & Requested set of slice data plane functions \\
$N_{v}$ & Requested set of slice VNFs $\left(N_{c} \cup N_{d}\right)$ \\
$L_{v}$ & Requested virtual links of a slice \\
$R^{i}$ & Requested CPU resource by a VNF $i$ \\
$R^{i j}$ & Requested BW resource between VNF $i, j$ \\
$d_{E 2 E}$ & Requested End-to-End delay \\
$K_{r e l}^{c}$ & Requested intra-slice isolation for Control Plane \\
$K_{r e l}^{d}$ & Requested intra-slice isolation for Data Plane \\
$\gamma^{c}$ & Requested inter-slice isolation for Control Plane \\
$\gamma^{d}$ & Requested inter-slice isolation for Data Plane \\
$u_{k f}^{i j}$ & Indicates the assignment of VNF $i$ to EPC node $k$ \\
\hline
\end{tabular}




\subsubsection{General Constraints}

1. Slice Assignment, Placement and Resource Budget

$$
\sum_{k \in N_{p}} u_{k}^{i}=1 \quad \forall i \in N_{v}
$$

$$
\sum_{i \in N_{v}}\left(R^{i}+\sigma_{k}\right) u_{k}^{i} \leq \sigma_{k}^{\max } \quad \forall k \in N_{p}
$$

$$
\sum_{(i, j) \in L_{v}}\left(R^{i j}+\sigma_{e f}^{i j}\right) y_{e f}^{i j} \leq \sigma_{e f}^{\max } \quad \forall(e, f) \in L_{p}
$$

$$
\sum_{i \in N_{v}} R^{i} \leq \sum_{k \in N_{p}}\left(\sigma_{k}^{\max }-\sigma_{k}\right)
$$

$$
\begin{aligned}
& \sum_{\substack{f \in N_{p} \\
(e \neq f)}}\left(y_{e f}^{i j}-y_{f e}^{i j}\right)=\left(u_{e}^{i}-u_{e}^{j}\right) \\
& \quad i \neq j, \forall(i, j) \in L_{v}, \forall e \in N_{p}
\end{aligned}
$$

$$
\begin{aligned}
& u_{k}^{i} \in\{0,1\} \quad \forall k \in N_{p}, \forall i \in N_{v} \\
& y_{\text {ef }}^{i j} \geq 0 \quad \forall(i, j) \in L_{v}, \forall(e, f) \in L_{p}
\end{aligned}
$$

The constraint (6.2) ensures that each VNF is assigned to exactly one server. Constraints (6.3) and (6.4) guarantee that allocated VNF resources do not exceed the physical servers' processing capacity and link bandwidth, respectively. A slice CPU demand should not exceed the remaining CPU capacity of the 
entire system. This is ensured by constraint (6.5) since the partial allocation of a slice is not the desired behavior. The conservation of flows, i.e., the sum of all incoming and outgoing traffic in the physical nodes that do not host VNFs should be zero is enforced by the constraint (6.6), and this constraint also ensures that there is a path between VNFs. Constraints (6.7) and (6.8) ensure that $u_{k}^{i}$ and $y_{e f}^{i j}$ are binary and integer, respectively.

\section{End-to-End Delay:}

$$
\begin{aligned}
& \left(\sum_{(i, j) \in L_{v}} \sum_{\substack{(e, f) \in L_{p} \\
e \neq f}} T_{e f} y_{e f}^{i j}+\sum_{i \in N_{v}}\left(\Delta^{i}+\sum_{k \in N_{p}} \Delta_{k} u_{k}^{i}\right)\right) \leq d_{E 2 E} \\
& T_{e f}=\frac{\sigma_{e f}}{\sigma_{e f}^{\text {max }}} \delta+T_{\text {ef,init }} \quad \forall(e, f) \in L_{p}
\end{aligned}
$$

Constraint (6.9) guarantees end-to-end delay for a slice in the current network state. End-to-end delay includes link delay $\left(T_{e f} y_{e f}^{i j}\right)$, VNF processing delay $\left(\Delta^{i}\right)$, and physical node processing delay $\left(\Delta_{k} u_{k}^{i}\right)$. Each time when a virtual link $(i, j) \in L_{v}$ is assigned to a physical link $(e, f) \in L_{p}$, it increases $T_{e f} \cdot T_{e f}$ is a function of link utilization, and it is calculated using eq. (6.10), where $T_{\text {ef,init }}$ is the initial delay assigned to the link $(e, f) \in L_{p}$ and $\delta$ is the maximum increase in delay. We explain in section $\S 6.5 .4$ how these values are calculated empirically. 


\subsubsection{Security-related Constraints}

\section{Intra-Slice Isolation}

$$
\begin{aligned}
& \sum_{i \in N_{c}} u_{k}^{i} \leq K_{r e l}^{c} \quad \forall k \in N_{p}, K_{r e l}^{c}=1,2,3 \ldots \\
& \sum_{i \in N_{d}} u_{k}^{i} \leq K_{r e l}^{d} \quad \forall k \in N_{p}, K_{r e l}^{d}=1,2,3 \ldots
\end{aligned}
$$

It might be required to have different levels of intra-slice isolation for control and data plane. In constraints $(6.11), K_{r e l}^{c}$ and $K_{r e l}^{d}$ ensure the intra-slice isolation for control plane and data plane respectively. Intra-slice isolation can improve the availability of a slice. For instance, if all components are hosted on one host, then a DDoS on one component could lead to the complete unavailability of the slice as well as longer migration time (if required). However, if there was some level of isolation, then DDoS on a single component might not result in complete unavailability, and the operator would not have to migrate the entire slice but some components that could result in faster recovery.

\section{Inter-Slice Isolation}

$$
\begin{gathered}
\sigma_{k} u_{k}^{i}=0 \Longleftrightarrow \gamma^{c}=1 \quad \forall k \in N_{p}, \forall i \in N_{c}, \gamma^{c} \in\{0,1\} \\
\sigma_{k} u_{k}^{i}=0 \Longleftrightarrow \gamma^{d}=1 \quad \forall k \in N_{p}, \forall i \in N_{d}, \gamma^{d} \in\{0,1\}
\end{gathered}
$$

Inter-slice isolation could provide more security in a multi-tenant environment. Constraint (6.12) provides the required hardware inter-slice isolation. In the allocation scheme, it might be required to have inter-slice isolation between control plane function (for security reasons) but not in the data plane functions or vice versa. Inter-slice isolation for control plane or data plane is only provided 
when $\gamma^{c}=1$ or $\gamma^{d}=1$, respectively. $u_{k}^{i}=1$ indicates the possible assignment of VNF $i$ to server $u$, and if we assume that $\gamma^{c}=1$ and $\gamma^{d}=1$ then by multiplying $\sigma_{k}$ (which indicates current CPU load of the server) with $u_{k}^{i}$, we can determine that if there were any previous VNFs allocated on the server $u$. If $\sigma_{k} u_{k}^{i}>0$, then slices have been allocated previously; otherwise, inter-slice allocation possible. We note that our inter-slice isolation does not provide bandwidth isolation.

\section{Secure Allocation}

$$
u_{k}^{i}=1 \rightarrow \alpha_{k}=\beta^{i} u_{k}^{i} \forall k \in N_{p}, \forall i \in N_{v}
$$

The secure allocation constraint allows the allocation of a slice that requires a secure environment ${ }^{1}$. To realize the secure allocation, some hypervisors are tagged as secure, represented by an integer variable $\alpha_{k}$ (i.e., network will be divided into multiple security zones), and slice components that require secure allocation are identified by an integer variable $\beta^{i}$. The secure allocation might be required for some components (e.g., control plane functions ) to be allocated at a predefined secure hypervisor(s). It will restrict the allocation of VNFs that require secure allocation to a secure hypervisor and everything else to the remaining hypervisors. An example of the secure allocation could be that the Unified Data Management function needs to be allocated in a predefined secure zone, and it cannot share the physical resources with the VNFs that do not request secure allocation. This constraint will guarantee such an allocation scheme.

\footnotetext{
${ }^{1}$ An example of a relatively secure environment for a user who is outside the organization's network could be Demilitarized Zone (DMZ) (relatively less secure) vs. internal network (relatively more secure).
} 


\subsection{DDoS Mitigation: Evaluation and Results}

The implementation of a complete $5 \mathrm{G}$ network is still in the early stages, and evaluating a fine-grained network slicing solution like ours in a 5G network is very challenging. There are very few testbeds and even fewer available to academia for testing and experimentation.

\subsubsection{Experiment Setup}

To evaluate our solution, we developed a testbed using seven physical servers. We use five servers to allocate slices, one as a DDoS node and one as a client, as shown in Fig. 6.2. The hardware specifications of the nodes are listed in table 6.2. In each of our experiments, we deploy 50 core network slices, each slice consisting of three components (network functions). AMPL [51] is used to model the optimization algorithm, and CPLEX 12.9.0.0 is used as a MILP solver. OpenVZ [52] is used for virtualization. It is an open-source container-based virtualization platform. OpenVZ allows each container to have a specific amount of CPU, RAM, and HDD. Each container (which hosts one VNF) performs and executes like a stand-alone server. We have installed the CentOS 6 [53] operating system in every container. We used Linux Traffic control $(t c)$ [54] to allocate bandwidth for each container.

Table 6.2: DDoS Experiment Topology Hardware Specification

\begin{tabular}{c|c}
\hline Server(s) & Hardware Specs \\
\hline PS1 to PS3, and A1 & CPU: 10GHz, RAM: 8GB, Bandwidth: 1Gbps \\
\hline PS4 and PS5 & CPU: 22.4GHz, RAM: 8GB, Bandwidth: 1Gbps \\
\hline \hline
\end{tabular}




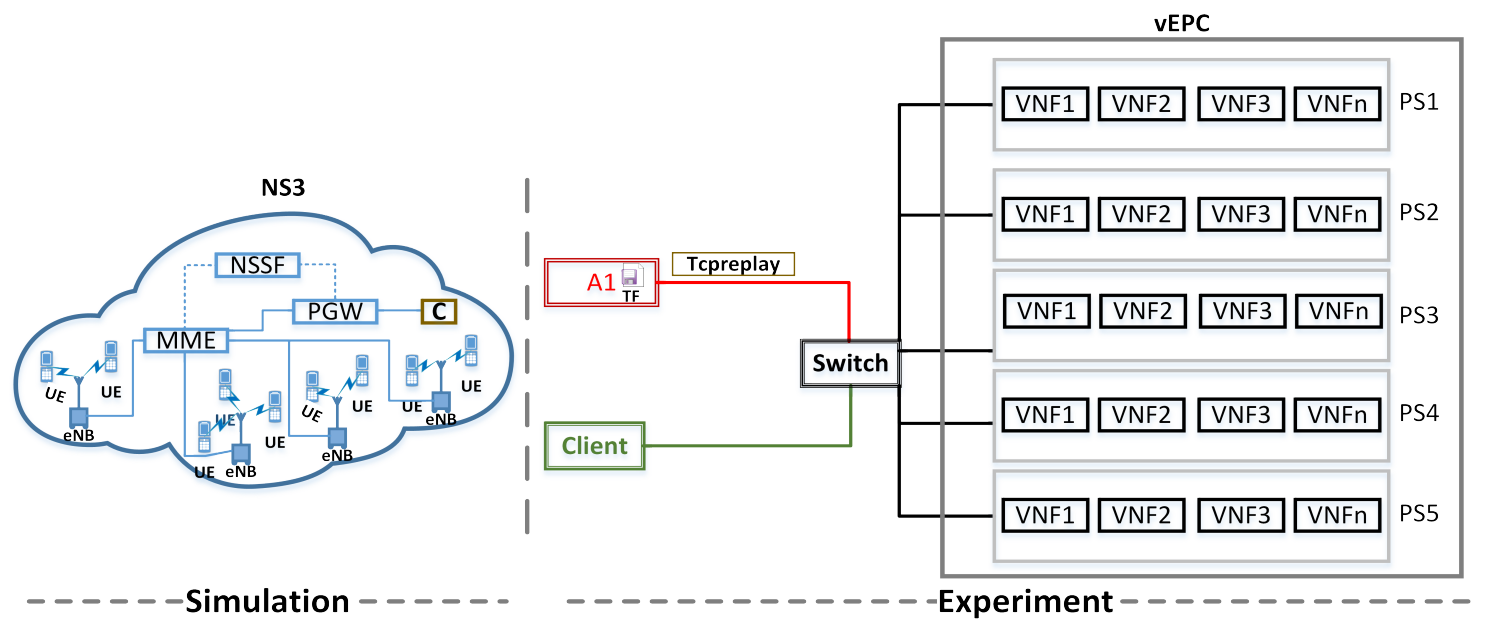

Figure 6.2: Evaluation Setup. The components used in the ns3 simulation are UE, eNB, MME, PGW and $\mathbf{C}$ is a traffic trace collection node. PS1-PS5 are the slice allocation servers, and A1 is a DDoS node. TF (pcap) is the traffic trace file collected in the simulation at $\mathbf{C}$ and replayed at A1 using tcpreplay

\subsubsection{Combining simulations with the use of testbed}

As demonstrated in Fig. 6.2, our evaluation setup uses a combination of a simulation setup and an experimental testbed. We perform our evaluation in two steps. In the first step, we use ns3 [55] to simulate the radio part of the 5G network where 320 mobile nodes generate UDP traffic and send it to the node $\mathbf{C}$ (see Fig. 6.2). At node C, we collect all traffic traces destined for the slices in a pcap file. This trace file collected from the simulation is used for all subsequent experiments that happen in the second step. In the second step which is performed on the testbed, we replay pcap file at servers A1 and A2 using tcpreplay [56] to perform DDoS attack on the slices. The two DDoS attack nodes A1 and A2 are used for a high bandwidth DDoS attack (at approximately $200 \mathrm{Mb} / \mathrm{s}$ ).

The reason for using the two-step approach is that we faced some challenges with scaling the experiments. The combination of a large amount of traffic generated by UEs and the emulated link between PGW and the rest of the network (slices), it 
became infeasible to perform DDoS using this setup. There was too much overhead in terms of processing requirements and network traffic. Therefore, we collected traffic traces at node $\mathbf{C}$ and used tcpreplay at A1 and A2 to perform DDoS attack.

For 5G-specific components, we used ns3 [55] with mmWave module [57] to simulate the 5G RAN network and used emulated link from PGW to connect the slices. We were able to implement Network Slicing Selection Function (NSSF) to select the slices based on the Network Slice Selection Assistance Information (NSSAI) received from the UEs or assign NSSAI to the UEs if it is unknown. In our ns3 implementation, if a UE does not know the NSSAI, the NSSF will assign the NSSAI, and it will redirect traffic to the correct slice (UEs-MME-NSSF-PGW-C). After the NSSAI assignment, the UEs will bypass the NSSF and directly go to the destination slice (UEs-MME-PGW-C).

\subsubsection{Test Application}

To test the impact of the different attacks, we measure specific performance parameters for a test application that we implemented on the testbed. We implemented a simple authentication protocol. In our implementation, we assume that the client (Green node in Fig. 6.2) already has an NSSAI, and it directly sends traffic to a specific slice. In our experiments, all slices can receive Transmission Control Protocol (TCP) and User Datagram Protocol (UDP) traffic. We note that we are using SEAF, AUSF, and HSS to describe our authentication mechanism's basic function. These network functions are not complete the implementation according to the 3GPP recommendations. Our HSS database has 1000 records, and it uses a binary search algorithm.

The messaging sequence of the authentication procedure is listed here and is also shown in Fig. 6.3. 
1. A client sends an authentication request to the slice SEAF.

2. SEAF performs preprocessing and forwards it to the AUSF of the slice.

3. AUSF forwards the request to the HSS and waits for the response.

4. The HSS performs a database search

5. HSS sends the result of the search to the AUSF

6. AUSF sends success or failure message to the SEAF based on information received from the HSS

7. SEAF forwards the information to the client

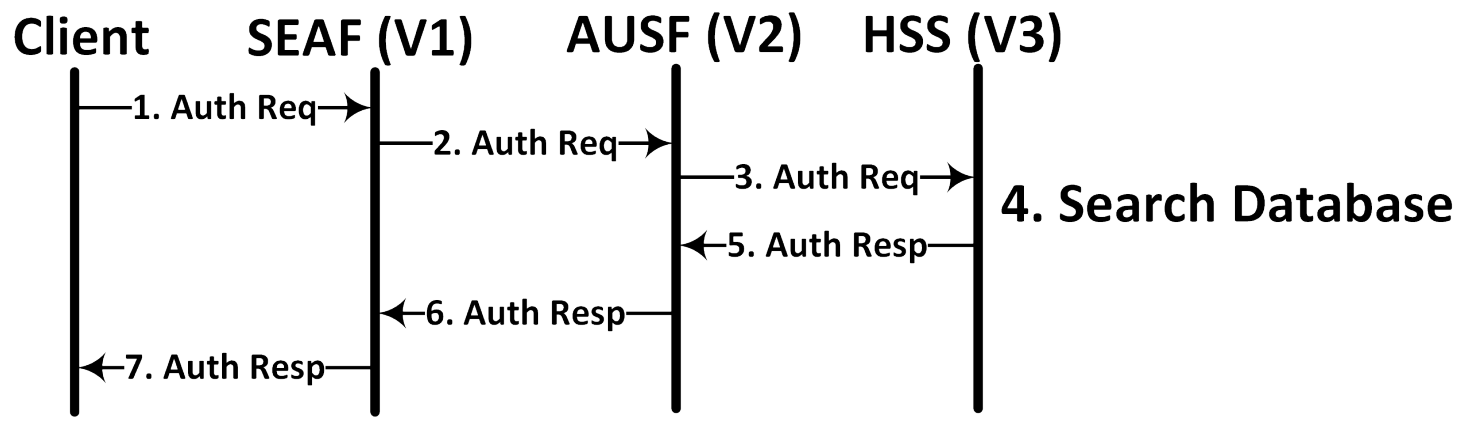

Figure 6.3: Flow diagram of the authentication procedure used in the experiments (V1, V2 and V3 represents virtual network functions)

\subsubsection{Evaluation Methodology}

To evaluate the performance impact of DDoS attack on slices, we collected response time and Round-Trip Time (RTT) between the client and the slice and the average slice bandwidth available to the client. We used Ping between the client and slice S1 (SEAF) to calculate the RTT. To calculate the average available bandwidth for the entire slice, we measured bandwidth between client and slice S1 (SEAF) and between 
the slice S1's components using iperf [58]. The slice $\boldsymbol{S} \boldsymbol{1}$ is providing authentication service to a client and the slice $\boldsymbol{S}_{\boldsymbol{4}}$ is under DDoS attack.

We empirically calculated the values for $T_{\text {ef,init }}$ and $\delta$ (eq. 6.10) by measuring RTT between client and slice S1's components and between different components of the slice S1. We measure RTT between client and slice S1 as well as between different components of slice S1 when there is almost no traffic in the network and take an average of all collected data to estimate $T_{\text {ef }}$, init. To estimate the value of $\delta$, we increase the utilization of all available links for slice S1 to approximately $100 \%$ and once again calculate RTT. We take an average of all the collected data, and it gives us a value of $\delta$. The rest of the parameters used in the experiment are listed in Table 6.3 .

Table 6.3: Inter-slice isolation DDoS experiment parameters

\begin{tabular}{r|l}
\hline Parameter & Value \\
\hline$N_{p}$ & 5 \\
$\sigma_{k}^{\text {max }}$ & $10.0 / 22.4 \mathrm{GHz}$ \\
$K_{r e l}$ & $1-3$ \\
$N_{v}$ & 3 \\
$R^{i j}$ & $40-60 \mathrm{Mbps}$ \\
$R^{i}$ & $0.4-1.4 \mathrm{GHz}$ \\
$\Delta^{i}$ & $0.1-0.8 \mathrm{~ms}$ \\
$\Delta_{k}$ & $0.5 \mathrm{~ms}$ \\
$\delta$ & $3.5 \mathrm{~ms}$ \\
$T_{e f}$, init & $0.13 \mathrm{~ms}$ \\
\hline Total Slice Requests & 50 \\
\hline
\end{tabular}




\subsubsection{Results and Discussion}

In this section, we discuss our experimental results. We devised two attack scenarios to realize the threat we described in section $§ 6.2 .3$. In both scenarios, the client is sending approximately ten authentication requests per sec to the slice S1 (SEAF), and the majority of the network traffic is directed towards either S1 or S4. To get a baseline (i.e., without isolation) results for comparison, we used a Greedy Algorithm (GA). In the greedy algorithm, the slices are allocated on a First Come First Serve basis. Once the PS1 is fully utilized, we start allocating slices to the next server and so on. In the figures $G A$ represents the results of greedy allocation under DDoS attack. Results for the different levels of intra-slice isolation are represented by $K_{\text {rel }}=1, K_{\text {rel }}=2$, and $K_{r e l}=3$. Inter-slice isolation results are represented by $I S I$ and NoDDoS shows the results of NoDDoS (baseline result without any DDoS attack).

We repeat every experiment three times. For every new value of $K_{r e l}$ and interslice isolation, a new optimization solution is calculated, and slices are reallocated accordingly before the corresponding experiments are run. We note that secure allocation constraint (eq. 6.13) is not enabled for both scenarios, and no hypervisor(s) are reserved for secure allocation.

For each value of $K_{r e l}$, we repeat every experiment three times. For every new value of $K_{\text {rel }}$, a new optimization solution is calculated, and slices are reallocated accordingly before the corresponding experiments are run. In the previous Chap. 5, we discussed the overall CPU and bandwidth utilization of the system, and average solver runtime for different levels of intra-slice isolation.

\subsubsection{DDoS flooding attack}

In evaluating the DDoS flooding attack scenario [47], the slice S4 was targeted (attack

details are in section $\$ 6.2 .3 .1$ ). Our client node is sending authentication requests to 
slice S1, and we use ns3 UDP traffic traces to launch a DDoS attack from A1 on slice S4. In this experiment, $\mathrm{CPU}$ is not a bottleneck. Fig. 6.4 shows the response time at the client. We can see a high response time for Greedy Algorithm (GA) and lowest for Inter-Slice Isolation (ISI). When there is no isolation present between slices, all the components of different slices share the same physical link. The Linux Traffic Controller $t c$ configures the kernel packet scheduler for all the virtual network functions (VNF) allocated on the same machine and, in this case, a total of six components for both slices (for simplicity, we can ignore the other slices for our discussion). When slice S4 is under DDoS, we can see a significant impact on the response time of the slice S1's client. However, the effect is gradually reduced when $K_{\text {rel }}=1, K_{\text {rel }}=2$ and $K_{\text {rel }}=3$. Inter-slice isolation (ISI) results in a significant improvement in the response time.

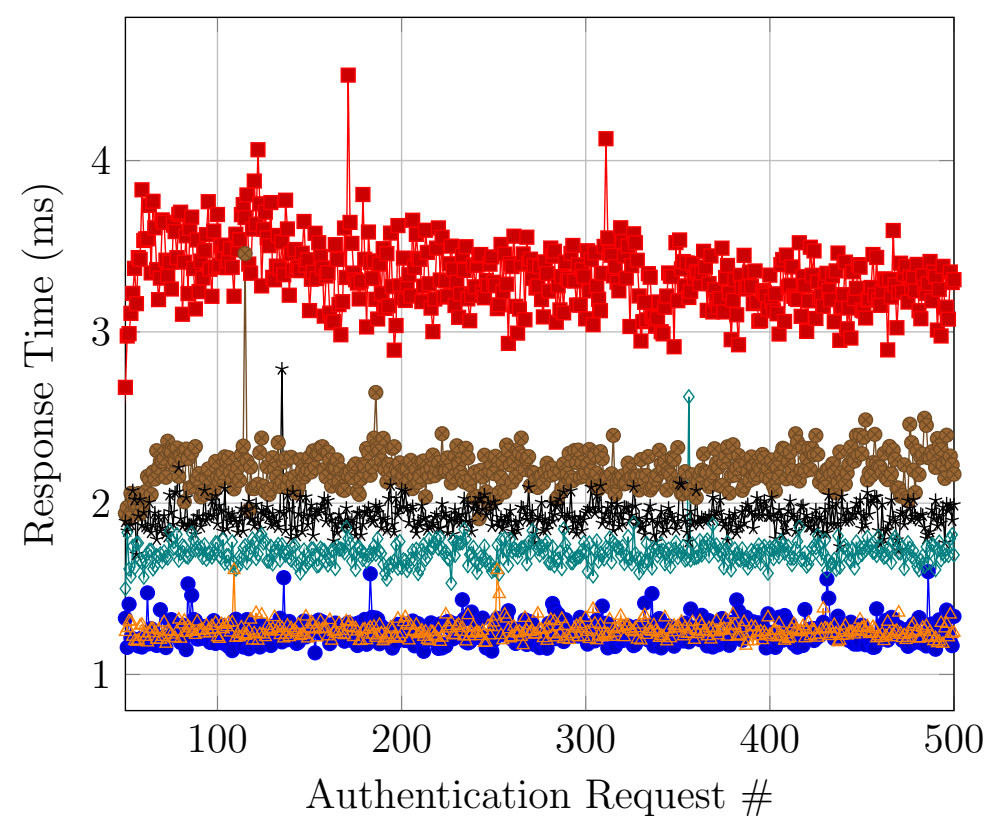

$$
\begin{array}{ll}
\bullet \mathrm{NoDDoS} \rightarrow K_{\text {rel }}=1 \leadsto K_{\text {rel }}=3 \\
\rightarrow \mathrm{GA} \rightarrow \star K_{\text {rel }}=2 \triangleleft \quad \text { ISI }
\end{array}
$$

Figure 6.4: Response Time for flooding DDoS attack scenario 


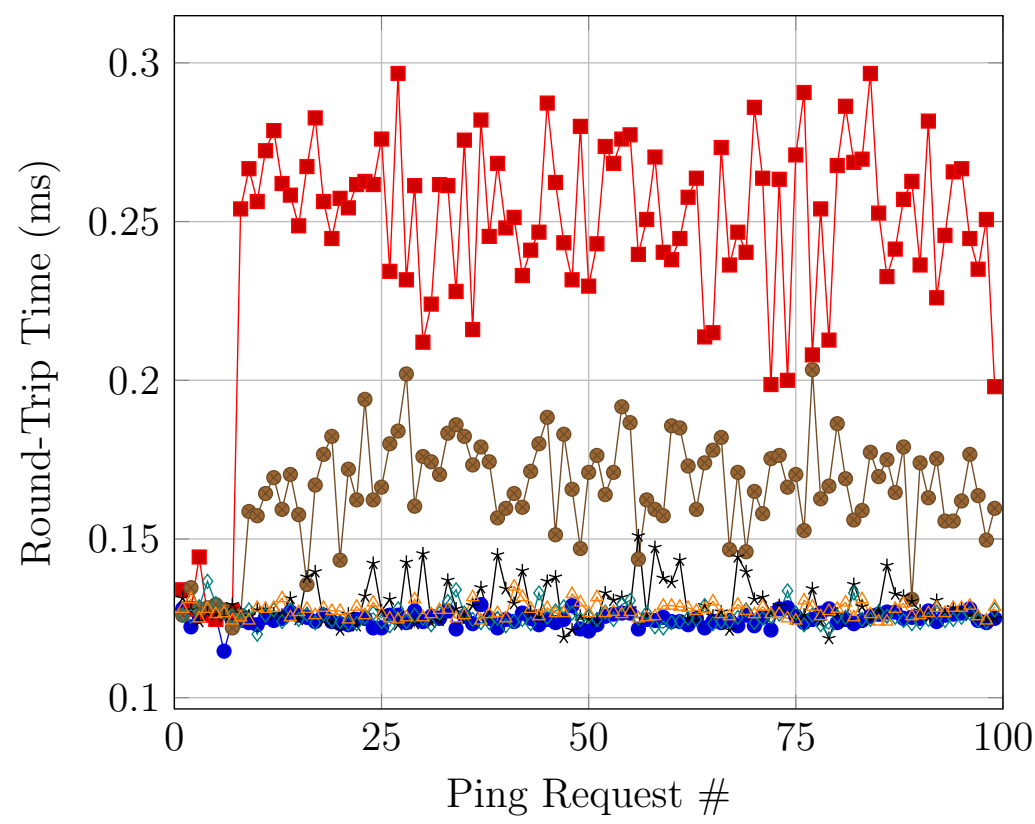

$-\mathrm{NoDDoS} \bullet K_{r e l}=1 \multimap K_{r e l}=3$
$-\mathrm{GA} * K_{r e l}=2 \backsim \quad$ ISI

Figure 6.5: Round-Trip Time for flooding DDoS attack scenario

The main reason is that a packet spends less time waiting in the kernel queue for each VNF as compared to the $G A$ case. For instance, in the $G A$ scenario, there will be a minimum of six queues at the kernel (one for each VNF). Whereas, when $K_{r e l}=1$, $K_{r e l}=2$, and $K_{r e l}=3$, the minimum number of queues will be reduced from six to two or three. Another factor that improved the client's response time is that there are multiple physical links available for the slice S1 to communicate with each other and the client. We see a trade-off (in terms of link delay) of using $K_{r e l}=1, K_{r e l}=2$, and $K_{r e l}=3$ because there is a link delay between the slice components. For instance, at $K_{\text {rel }}=1$ each component is hosted at a different hypervisor, we see an increased response time as compared to $K_{\text {rel }}=2$ and $K_{\text {rel }}=3$. Another factor that impacted the response time for the $K_{r e l}=2$ and $K_{r e l}=3$ is that which component of the slice is hosted at which hypervisor. For example, if V2 is allocated on a different hypervisor 
then V1 or V3, there will be an additional delay $\left(K_{r e l}=2\right)$ as compared to the when V1 and V2 or V2 and V3 are allocated on the same hypervisor $\left(K_{r e l}=3\right)$.

We calculated RTT from the client to slice S1 (SEAF), as shown in Fig. 6.5. We can see a significant advantage of our isolation solution at all levels of $K_{\text {rel }}$ compared to GA. However, the RTT is not as high as the response time (i.e., Fig. 6.4). The RTT is calculated only from the client to the $\mathrm{S} 1$ (SEAF), but in the response time, an authentication request passes through all three nodes that could add a significant delay (i.e., see authentication message flow in Fig. 6.3). There is an increase in RTT for the $K_{\text {rel }}=1$ as compared to the $K_{r e l}=2$, and $K_{\text {rel }}=3$ because V1 of both slices are allocated on the same hypervisor.

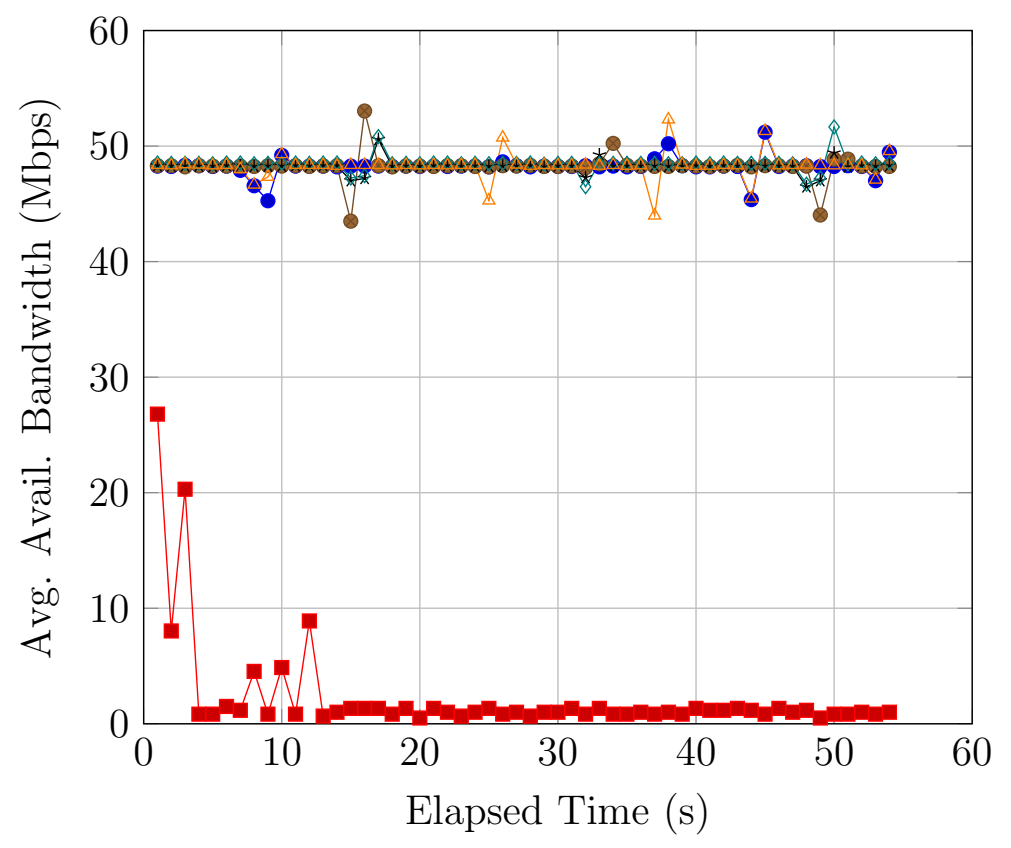

$$
\begin{aligned}
& \bullet \mathrm{NoDDoS} \bullet K_{\text {rel }}=1 \leadsto K_{\text {rel }}=3 \\
& -\mathrm{GA} * K_{r e l}=2 \triangleleft \quad \text { ISI }
\end{aligned}
$$

Figure 6.6: Average available bandwidth (BW) for the slice in flooding DDoS attack scenario

Fig. 6.6 shows the average available bandwidth in the entire slice S1. We measured 
bandwidth using iperf between client and S1 (SEAF) and between slice S1's virtual network functions (we only plot average bandwidth). The slice $\mathrm{S} 1$ is allocated $50 \mathrm{Mb} / \mathrm{s}$ bandwidth. As we can see, bandwidth for slice S1 drops to approximately $1 \mathrm{Mb} / \mathrm{s}$ for $G A$ and these results also provide some insight for such high response time (Fig. 6.4) and RTT (Fig. 6.5). There is significantly less bandwidth available in the entire slice S1. However, our isolation solution guarantees a high percentage of the allocated bandwidth.

\subsubsection{Slice-initiated attack}

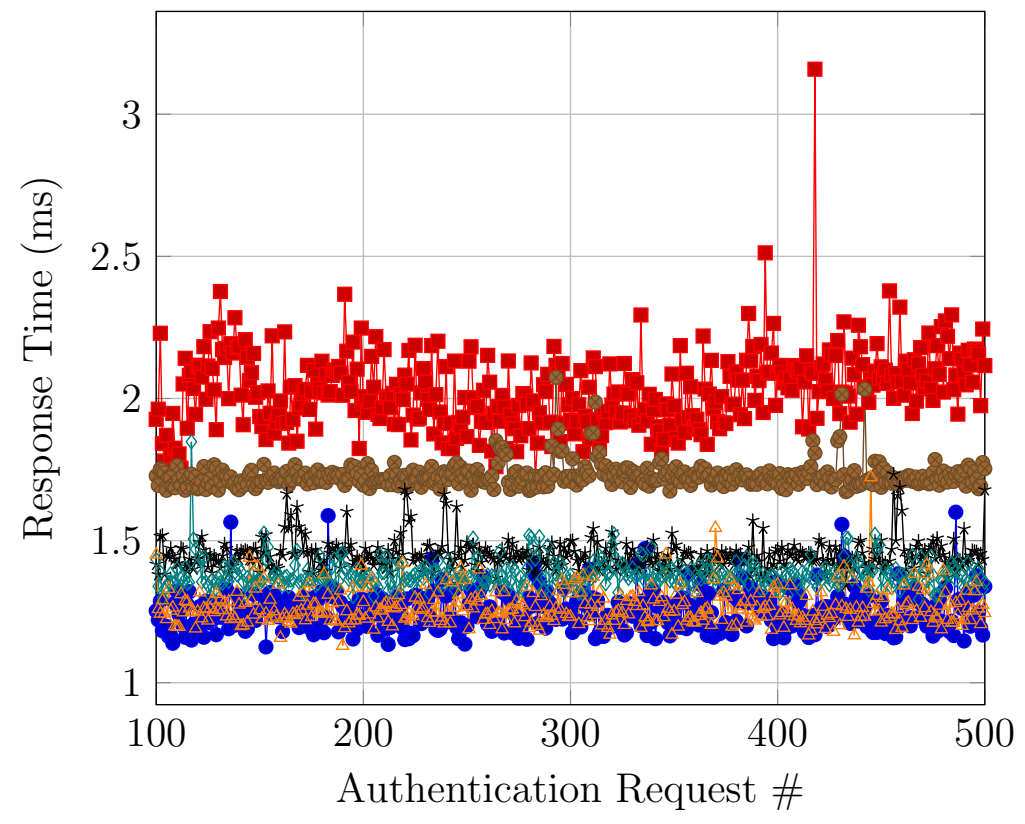

$$
\begin{aligned}
& \bullet \operatorname{NoDDoS} \bullet K_{r e l}=1 \multimap K_{r e l}=3 \\
& \rightarrow \text { GA } \rightarrow K_{r e l}=2 \backsim \quad \text { ISI }
\end{aligned}
$$

Figure 6.7: Response Time for slice-initiated attack scenario

In the slice-initiated attack scenario (see section $§ 6.2 .3 .2$ for more details), we overload the CPU of slice S4 using a simple Python script and measure the impact on slice S1. There is no flooding DDoS attack happening in this scenario. The impact of 
the CPU bottleneck depends on various factors, e.g., applications running on the host system and within containers (VMs), the host operating system, and virtualization platform. In our experiments, we tried to keep the impact of the factors mentioned above to a minimum ${ }^{2}$ and measure the response time and RTT for the client as shown in Fig. 6.7 and Fig. 6.8, respectively. As shown, the response time is relatively high for the client when there is no isolation $(G A)$. Whereas, our isolation solution for all levels of $K_{r e l}$ and ISI shows a minimum impact of sustained high CPU utilization. Only $K_{r e l}=1$ shows a slight increase in the response time due to link delays. The minor variation between response times for different levels of $K_{r e l}$ could be due to the factors we mentioned earlier.

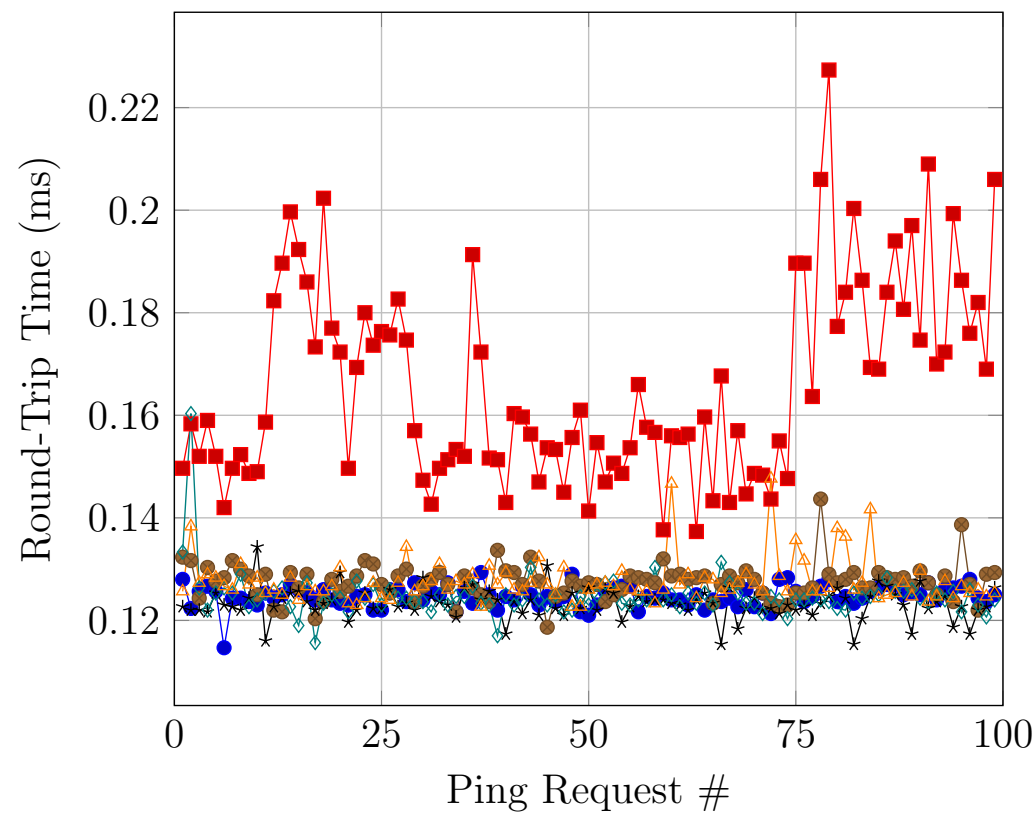

$$
\begin{aligned}
& \rightarrow \text { NoDDoS } \bullet-K_{\text {rel }}=1 \multimap K_{\text {rel }}=3 \\
& \rightarrow \quad \text { GA } \rightarrow-K_{\text {rel }}=2 \triangleleft \quad \text { ISI }
\end{aligned}
$$

Figure 6.8: Round-Trip Time for slice-initiated attack scenario

\footnotetext{
${ }^{2}$ We do not launch any additional applications between different experiments as well as at the beginning of each experiment, we measure CPU and RAM utilization to confirm that they are at a similar level.
} 
For RTT, we see much more variation in the case of $G A$. The reason could be that when a Ping request arrives at the destination VNF, sometimes the CPU might be less loaded, and it can process the request sooner, and other times it might be more loaded and respond more slowly. However, the CPU bottleneck has a minimum impact on all levels of $K_{r e l}$ and $I S I$. The impact of slice-initiated attacks could have been worse if other hosted slices were using some CPU intensive applications. The results for both scenarios are consistent with our previous work [59].

\subsection{Performance Evaluation of the Optimization Model}

The performance of the optimization has an impact on our solution. We dedicate this section to present the results of studying the performance of the optimization using simulation. We study the performance impact of enabling security constraint ( eq. 6.13). MATLAB is used to calculate the optimization solution and perform pre/postprocessing of data. The preprocessing of data involves reading current network topology, slice requests, and updating the optimization model. In the post-processing, we update the network topology after a slice is allocated. AMPL is used to model optimization algorithm, and CPLEX 12.9.0.0 is used as a MILP solver. The optimization model is evaluated on Intel Core i7-8700 $3.2 \mathrm{GHz}$ with 32 GB RAM. We simulate 200 servers to allocate 500 slice requests, as shown in Fig. 6.9 (we used similar topology to [50]). Other parameters used for the evaluation are listed in Table 6.4. In our performance evaluation, we vary the intra-slice isolation level using the $K_{\text {rel }}$ parameter. This parameter provides the upper limit for how many VNFs can be placed on

one physical server. For $K_{\text {rel }} 1$ to 10 , all slices request the same level of intra-slice 
isolation. In addition to slice isolation, the model guarantees the requested computing resources, bandwidth resources, and end-to-end delay for a slice in the current network state. After allocating each slice, we update the network delays, remaining computing, and bandwidth resources.

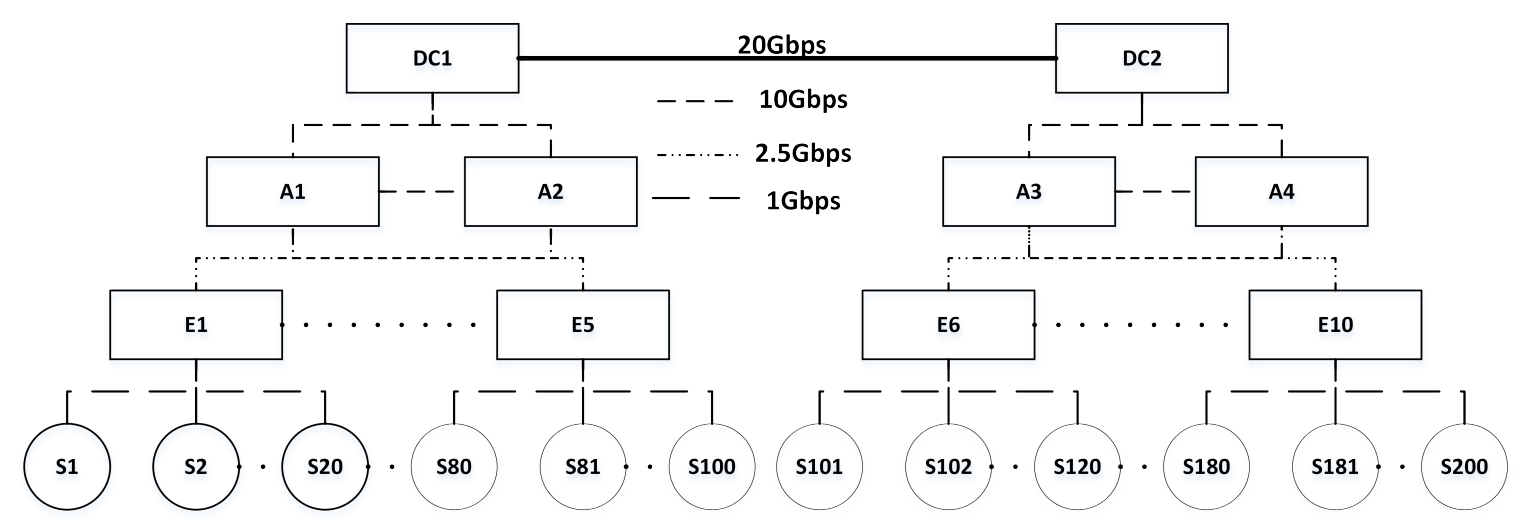

Figure 6.9: Simulation Topology

We evaluated two different scenarios. In the first scenario, the secure allocation constraint (eq. 6.13) is disabled. We call this scenario "Default" because this is the default behavior of the optimization model. The second scenario is called "Secure," in which we randomly select some VNFs to be allocated on secure servers (in our simulation, we only used two zones, i.e., secure and non-secure). For both scenarios and every intra-slice slice isolation level, we run the optimization model 3 times and plot the average. We note that inter-slice isolation is disabled for both scenarios (i.e., $\left.\gamma^{c}=0, \gamma^{d}=0\right)^{3}$.

Fig. 6.10 shows the allocation efficiency and the overhead of the optimization model. CPU utilization, bandwidth utilization, and requests accepted are shown in Fig. 6.10a, 6.10b and 6.10c, respectively. As we can see, relaxing the intra-slice

\footnotetext{
${ }^{3}$ Inter-slice isolation significantly reduces the allocation efficiency because if a slice is allocated on a hypervisor, then no other slice can be allocated on the same hypervisor. It could be required to provide the highest level of isolation but at the cost of allocation efficiency.
} 
Table 6.4: Secure allocation performance evaluation parameters

\begin{tabular}{r|l}
\hline Parameter & Value \\
\hline$N_{p}$ & 200 \\
$\sigma_{k}^{\max }$ & $25 \mathrm{GHz}$ \\
$K_{\text {rel }}$ & $1-10$ \\
$N_{v}$ & 10 \\
$R^{i j}$ & $40-60 \mathrm{Mbps}$ \\
$R^{i}$ & $0.55-1.6 \mathrm{GHz}$ \\
$\Delta^{i}$ & $0.2-0.6 \mathrm{~ms}$ \\
$\Delta_{k}$ & $0.2 \mathrm{~ms}$ \\
$\delta$ & $3.5 \mathrm{~ms}$ \\
$T_{e f}$, init & $0.13 \mathrm{~ms}$ \\
Total Slice Requests & 500 \\
\hline
\end{tabular}

isolation results in allocating more requests and better utilization of the available resources. In the Default allocation scenario, the optimization model can allocate more requests as compared to the Secure scenario due to two reasons. First, Default scenario does not take into consideration any secure allocation (eq. 6.13) requirements, and second, more physical resources are available for allocation. Whereas, in Secure scenario, some servers are reserved for only secure allocation (i.e., $12.5 \%$ of the servers are reserved for secure allocation and $10 \%$ of the VNF request secure allocation), which in turn results in reduced resources for the rest of the slice requests. An interesting result to note here is that bandwidth utilization is high for the Secure scenario (Fig. 6.10b), although fewer requests are allocated because VNFs of the slices are more spread across the network due to secure allocation constraint (eq. 6.13) as compared to the Default scenario.

We present the actual overhead incurred due to the optimization model for each 
request in terms of solver runtime, as shown in Fig. 6.10d. It is the time required by the CPLEX to find an optimal allocation solution for each slice request. As we can see, strict intra-slice isolation requirements $\left(K_{\text {rel }}=1\right.$ and $\left.K_{\text {rel }}=2\right)$ requires more time to find an optimal solution as compared to the relaxed intra-slice isolation requirements $\left(K_{r e l}=3\right.$ to $\left.K_{r e l}=10\right)$ in both scenarios. The Secure allocation (eq. 6.13) has higher solver runtime because there is an additional constraint for slice allocation that requires more $\mathrm{CPU}$ time.

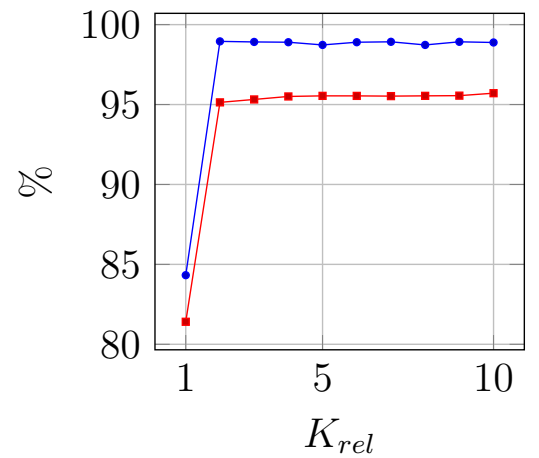

(a) CPU Utilization

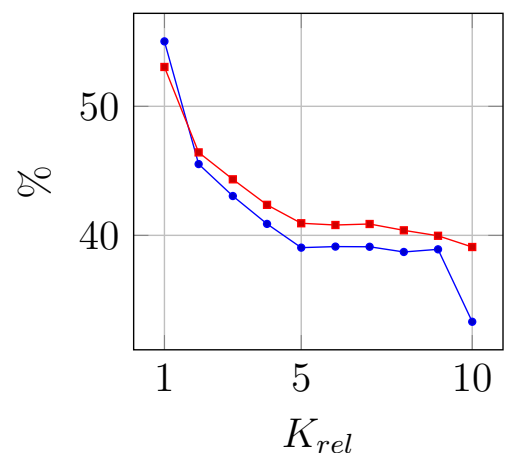

(b) Bandwidth Utilization

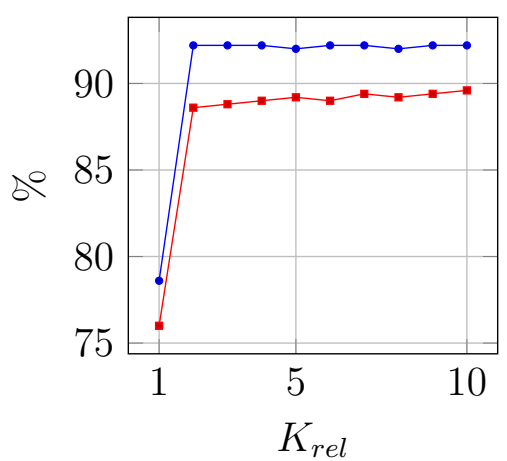

(c) Requests Accepted

\section{$\rightarrow$ Default $\rightarrow$-Secure}

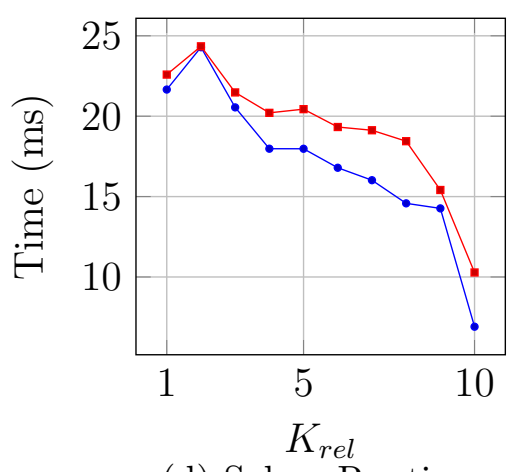

(d) Solver Runtime

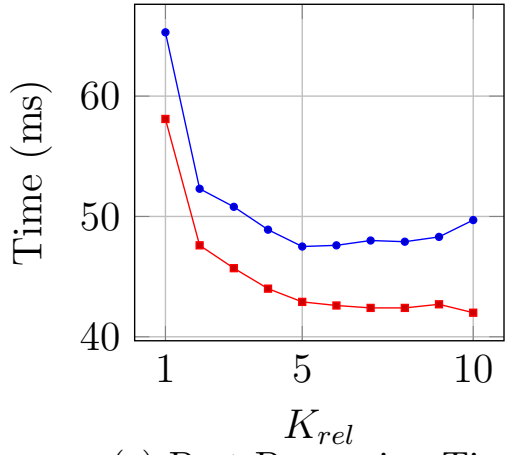

(e) Post-Processing Time

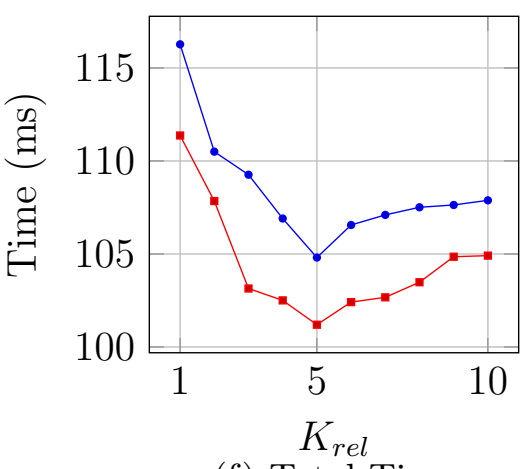

(f) Total Time

Figure 6.10: Performance evaluation for varying level of $K_{\text {rel }}$

The post-processing time is the time required to update the system statistics after allocating each slice, as shown in Fig. $6 \cdot 10 \mathrm{e}$. At $K_{\text {rel }}=1$, the post-processing is high 
due to the each VNF of a slice is allocated to a separate hypervisor, therefore requiring more system statistics updates. An interesting point to note here is that at $K_{r e l}=5$, the post-processing time is the lowest, and it gradually starts to increase for higher levels of intra-slice isolation. Because at higher intra-slice isolation levels, the VNF slices will start to spread more after a certain time. For instance, at $K_{\text {rel }}=10$, slices allocated at the start of the allocation process will have the lowest post-processing time. Because they will be allocated on the same hypervisor (bandwidth utilization will be low as well), but as the remaining overall system capacity reduces, the VNFs of each slice request will be spread across the system that will increase the postprocessing time and bandwidth utilization. However, the average post-processing time for all the allocated requests might be slightly skewed depending on the number of requests allocated with less (or more) system statistics updates. The Secure scenario has lower post-processing time as compared to the Default scenario. The main reason is that the requests accepted are higher for Default scenario that increases the average system statistics updates, which results in higher average post-processing time for the Default scenario.

We also measure the total time required to process each request, as shown in Fig. 6.10f. This time includes the times required to populate the data in AMPL, find a solution (i.e., CPLEX time), and post-processing time. The total time shows very similar behavior to the post-processing time.

\subsection{Current Limitations}

Worst-case scenario allocation: In our current solution, a worst-case scenario would be if a significant number of VNFs from different slices that require either high bandwidth or perform CPU intensive tasks (could be a combination of both) 
are allocated on the same physical host(s). The allocation scheme does not consider what type of VNFs are previously allocated on any specific host(s). So if they were of the resource-intensive type, and they happen to reach their maximum load at similar times, a DDoS attack on a slice at that time could pose significant mitigation challenges.

\subsection{Summary}

In our solution, We used slice isolation to reduce the impact of DDoS attacks on a simple network service (slice authentication). We evaluated our solution using a combination of simulation and an experimental testbed for flooding DDoS attacks and slice-initiated attacks. In both attack scenarios, complete slice isolation provides the best mitigation (compared to different levels of intra-slice isolation and GA). Whereas, at different intra-slice isolation levels $K_{r e l}=1, K_{r e l}=2$, and $K_{r e l}=3$ the impact of DDoS on the response time and round-trip time are significantly reduced as compared to the no isolation case $(G A)$. During the DDoS attack scenario, the average bandwidth available to the client is a fraction of the requested bandwidth, when there is no isolation. However, the impact of DDoS on average available bandwidth with our solution enabled is negligible.

We note that inter-slice isolation provides strong resource isolation. However, it can reduce the efficiency of resource utilization. Intra-Slice isolation provides better control over the trade-off between security, availability, and resource utilization. 


\section{Chapter 7}

\section{Proactive Allocation as Defense for Malicious Co-residency in Sliced 5G Core Networks}

66 As we've come to realize, the idea that security starts and ends with the purchase of a prepackaged firewall is simply misguided. 99

Art Wittmann

Network Slicing has been proposed to cope with the ever-growing demand for flexibility and scalability in 5G mobile network $[60,61]$. The recent advancements in Network Function Virtualization (NFV) have enabled next-generation mobile networks to employ concepts like network slicing to satisfy diverse requirements from various new applications [62]. The Next Generation Mobile Networks (NGMN) Alliance defined network slicing as running multiple services with different requirements such as performance, security, availability, reliability, mobility, and cost as an independent logical network on the shared physical infrastructure [3,60]. An end-to-end slice is created by pairing the RAN and core network slice, but the relationship between both slices could be 1-to-1 or 1-to-M [15,35]. 
One of the key requirements for network slicing is resource isolation between different slices $[60,62]$. However, guaranteeing resource isolation between slices that share the common physical infrastructure is challenging [3]. The sharing of common physical resources between slices could lead to information leakage and side-channel attacks $[63,64]$. The side-channel attacks can be used to determine co-residency and extract valuable information (e.g., cryptographic keys [65]) from the victim slices or perform Denial-of-Service attacks [59]. Several types of side-channel attacks can be used to determine co-residency by using different shared resources such as CPU cache, main memory, and network traffic [65]. In Chap. 6, we described and implemented the slice-initiated attacks, where the attacker tries to impact the performance of coresided slices by abusing the allocated resources. The precondition to such an attack is having co-residency with the target slice. Therefore, it is paramount to provide defense against malicious co-residency and minimize side-channel attacks.

In this chapter, our focus is to analyze the impact of optimization-based slice allocation on malicious co-residency. We also discuss additional measures that can be taken to further minimize the risk of co-residency.

\subsection{Related Work}

The next-generation mobile networks will have similar co-residency issues as cloud networks since both networks share some properties, i.e., shared resources and multitenancy. The work on the co-residency issue in the $5 \mathrm{G}$ network is currently limited due to its infancy. Therefore in this section, we describe some state-of-the-artwork on co-residency detection in cloud networks, which would still be applicable in the $5 \mathrm{G}$ network because both networks share virtualized infrastructure.

Network traffic is one of the shared resources that can be used to determine 
co-residency. In [66], A. Bates et al. used network traffic watermarking to detect coresidency with the victim. In the proposed scheme, the attacker launches multiple VM instances called FLOODER that communicate with the CLIENT, which is outside the cloud network. The CLIENT sends legitimate traffic to the target (victim) server that resides inside the cloud network. The FLOODER VMs flood the network with traffic to cause network delays, and the CLIENT analyzes these delays to determine which FLOODER is co-resided with the target server. Another aspect of network traffic can be exploited by analyzing Round Trip Time (RTT) to detect co-residency. Such a method is discussed by A. Atya et al. in [63]. In the proposed work, TCP handshake is used to measure the RTT (in some cases, ICMP was also be used) to determine co-residency. RTT is calculated from multiple sources and vantage points to increase the accuracy of detecting co-residency. A migration scheme was proposed to defend against co-residency attacks. An extension of their work is also discussed in $[67]$.

CPU cache-based side-channel attacks are commonly used to detect co-residency with the victim VM. Authors Y. Zhang et al. [68] used L2 cache to detect co-residency in the cloud environment. The objective of their works was to use side-channel to detect undesired co-residency. The basic idea of HomeAlone was to coordinate with other friendly VMs and analyze the cache usage to determine if there are any undesired VMs hosted on the same hypervisor. 


\subsection{Threat Model}

Assumptions: Our threat model assumes that network slicing is supported by the target network, the Evolved Packet Core (EPC) supports migration of the slice components, the slice operator or users can migrate the slice(s), on the servers, multitenancy is supported by the slice operator, adversaries do not know about the operators' allocation scheme, and adversaries can successfully determine the co-residency with the victim slice.

Adversary: The adversaries can launch multiple VMs and check for the coresidency with the victim. If co-residency is found then, the attacker could launch the next $\operatorname{attack}(\mathrm{s})$. If not, remove the slice and launch new ones and repeat the process. We assume that adversaries are not colluding, and the attacker's request rate is limited by the target system's request handling capabilities. The attacker can request a variable amount of resources (i.e., CPU and bandwidth)

\subsection{Simulation Setup}

MATLAB is used to calculate the optimization solution and perform pre/postprocessing of data. The preprocessing of data involves reading current network topology, slice requests, and updating the optimization model. In the post-processing, we update the network topology after a slice is allocated. AMPL is used to model optimization algorithm, and CPLEX 12.9.0.0 is used as a MILP solver. The optimization model is evaluated on Intel Core i7-8700 $3.2 \mathrm{GHz}$ with 32 GB RAM. We simulate 200 servers, as shown in Fig. 6.9 (we used same topology to section §6.6). Other parameters used for the evaluation are listed in Table 7.1. In our simulation, we vary the level of intra-slice isolation using the $K_{\text {rel }}$ parameter. This parameter provides the upper limit for how many VNFs can be placed on one physical server. For $K_{r e l}$ 
1 to 10 , all slices request the same level of intra-slice isolation. The average overall $\mathrm{CPU}$ utilization of the entire system is also restricted at $50 \%, 75 \%, 80 \%, 85 \%, 90 \%$, and $95 \%$ ( $\pm \Delta 0.5 \%)$. For each average CPU utilization (ACU), we vary the $K_{\text {rel }}$ (e.g., at average CPU utilization $50 \%, K_{\text {rel }}$ will be varies from 1 to 10$)$.

Table 7.1: Simulation Parameters

\begin{tabular}{r||l}
\hline Parameter & Value \\
\hline$N_{p}$ & 200 \\
$\sigma_{k}^{\text {max }}$ & $25 \mathrm{GHz}$ \\
$K_{\text {rel }}$ & $1-10$ \\
$N_{v}$ & 10 \\
$R^{i j}$ & $40-60 \mathrm{Mbps}$ \\
$R^{i}$ & $0.55-1.6 \mathrm{GHz}$ \\
$\Delta^{i}$ & $0.2-0.6 \mathrm{~ms}$ \\
$\Delta_{k}$ & $0.2 \mathrm{~ms}$ \\
$\delta$ & $3.5 \mathrm{~ms}$ \\
$T_{e f}$, init & $0.13 \mathrm{~ms}$ \\
Total Attacker Slice Requests & 500 \\
Target Slice & 50 \\
\hline
\end{tabular}

In each simulation, the attacker requests allocation of a slice and determines if there is a co-residency with the victim slice (i.e., one or more VNFs of the victim slice are allocated on the same hypervisor as the attacker). If co-residency is found, it is considered a success (we assume that attacker will move to the next step of their objective in the real world), and if no co-residency is found, it is considered a failure. In either case, we deallocate the attacker slice and request a new slice. We repeat this process 500 times and calculate the average success rate. The success is defined as 
if one or more VNFs of a victim slice are allocated on the same hypervisor as the attacker. We only generate 500 attacker requests once at the beginning of the simulation. Every 60 seconds, a legitimate slice is deallocated to simulate a more realistic scenario, and a new slice is allocated. The target slice is never deallocated.

\subsection{Results and Discussion}

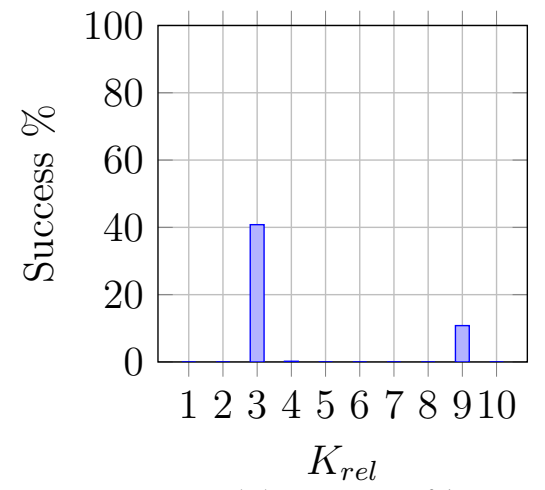

(a) ACU $50 \%$

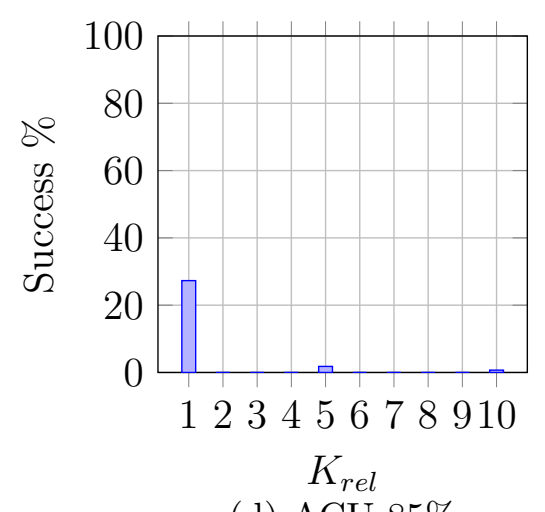

(d) ACU $85 \%$

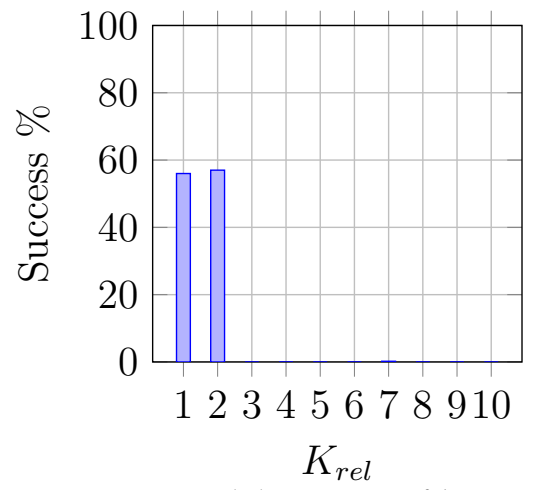

(b) $\mathrm{ACU} 75 \%$

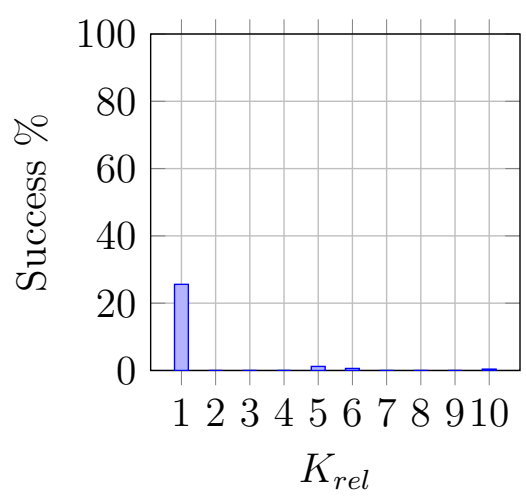

(e) $\mathrm{ACU} 90 \%$

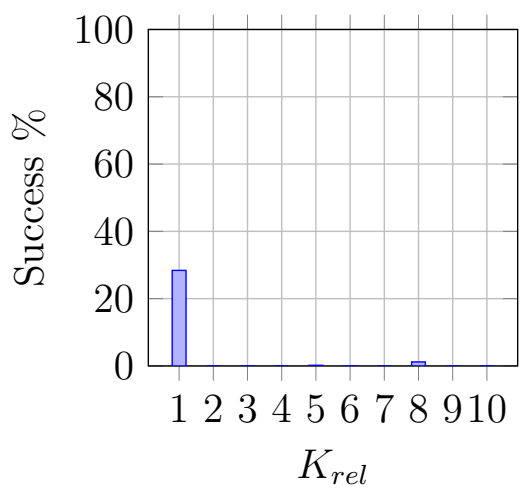

(c) $\mathrm{ACU} 80 \%$

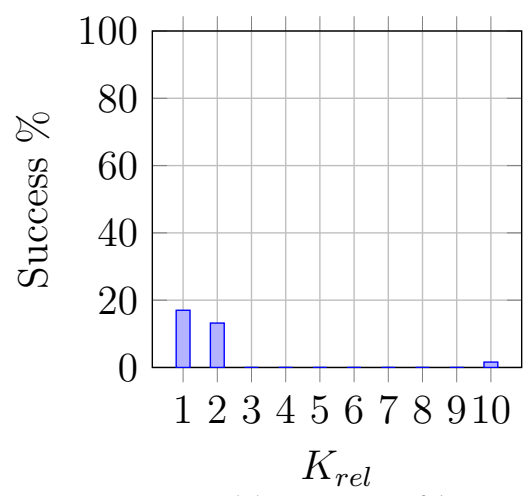

(f) ACU $95 \%$

Figure 7.1: Comparison of Co-Residency Success rate for different average CPU utilization

Fig. 7.1 shows the relationship between different levels of intra-slice isolation and the success rate of getting a co-residency with any network function of the target 
slice. In the figure, there is a relatively higher chance of getting co-residency when $K_{\text {rel }} \leq 3$ and lower ACU because of two reasons. First, at $K_{\text {rel }} \leq 3$, the network functions are more spread across the network, which increases the chances of getting co-residency with a specific target slice. Second, at relatively lower $\mathrm{ACU}^{1}$ there are more opportunities to get co-allocation. Whereas at higher $K_{\text {rel }} \geq 4$ and $A C U \geq 80 \%$, we see a significant decrease in the success rate of getting a co-residency. For instance, $K_{r e l}=1$ and $A C U=75 \%$, the success rate is $56 \%$ whereas $K_{\text {rel }}=1$ and $A C U=80 \%$, the success rate is only $29 \%$ (almost $50 \%$ reduction in success rate). At $K_{r e l} \geq 4$, the slice would have a certain degree of isolation as well as at $A C U>75$ present a more realistic scenario for the slice operator because the network resources will be better utilized.

\subsection{Defense}

There are a few methods that can be employed to reduce the threat of malicious co-residency.

- Detect anomalous behavior for slice allocation requests (e.g., monitor IP/MAC/unique user ID or other parameters) and take necessary preventive measures.

- If we assume that there is DDoS detection, then it would be possible to migrate the target slice to a different location (hypervisor). It could be a slice operator or the user (if allowed) that can migrate the slice.

- Limit the number of slice requests allowed perusers within a specified period and the total number of requests. Such a method could limit the requests generated

${ }^{1} A C U<50 \%$ does not yield meaningful results due to low resource utilization. Therefore, we have not shown those results here 
by the legitimate users, but if used in conjunction with the above methods, it should reduce the false positives ${ }^{2}$.

\subsection{Summary}

In this chapter, we presented an analysis of the impact of optimization-based slice allocation on malicious co-residency. Our optimization model inherently provides a proactive defense against malicious co-residency. The success rate of getting coresidency with the target slice decreases with the increase in $K_{r e l}$ levels and Average CPU Utilization of the system. The selection of $K_{r e l}$ depends on several factors, i.e., cost, security, and performance. For instance, if a slice requires higher level of DDoS protection then lower $K_{\text {rel }}$ might be required to provide high availability (e.g., $\left.K_{r e l}=1\right)$. Whereas to reduce the cost and the risk of malicious co-residency, a slice might require higher $K_{r e l}$ (e.g., $K_{r e l} \geq 4$ ). Another factor that could impact the selection of $K_{r e l}$ is the state of the slice operator's network (e.g., ACU). Therefore, the selection of $K_{r e l}$ greatly depends on the requirements of a slice and state of the operator's network. The natural defense against malicious co-residency comes at no additional computational cost to the network operator since the cost is already included in the slice allocation.

\footnotetext{
${ }^{2}$ We note that these proposed solutions require further investigation.
} 


\section{Chapter 8}

\section{Dynamic Slice Allocation Framework (DSAF)}

66 Security used to be an inconvenience sometimes, but now its a necessity all the time. 99

Martina Navratilova

Network slicing is a key to supporting different quality-of-service requirements for users and application in the 5G network. However, allocating network slices efficiently while providing a minimum guaranteed level of service in a mobile core is challenging. To address this question, in Chap. 6, we proposed an optimization model to allocate slices. It provided a static and manual allocation of slices. In this chapter, we extend our work to dynamically allocated slices. We propose a dynamic slice allocation framework for the 5G core network. The proposed framework provides user-interaction to request slices and any required services that need to run on a slice(s). It can accept a single or multiple allocation requests, and it dynamically allocates them. Additionally, the framework allocates slices in a balanced ${ }^{1}$ fashion

\footnotetext{
${ }^{1}$ Our solution will try to to make sure that average CPU allocation across all available hypervisors is approximately equal
} 
across available resources. We compare our framework with the First Come First Serve and First Available allocation scheme.

\subsection{DSAF: Dynamic Slice Allocation Framework}

To automate the process of slice allocation in the 5G core network, we propose a framework. In the proposed framework, slices can be allocated and deallocated dynamically. The Dynamic Slice Allocation Framework (DSAF) consists of five components as shown in Fig. 8.1.

Table 8.1: Example Slice CPU Requests Database

\begin{tabular}{c|c|c}
\hline Slice\# & VNF\# & CPU (GHz) \\
\hline \multirow{3}{*}{ S1 } & V1 & 0.5 \\
& V2 & 0.6 \\
& V3 & 0.4 \\
\hline & V1 & 0.35 \\
& V2 & 0.55 \\
& V3 & 0.75 \\
\hline \hline
\end{tabular}

Table 8.2: Example Slice CPU Allocations Database

\begin{tabular}{c|c|c}
\hline Slice\# & VNF\# & Allocated Hypervisor \\
\hline \multirow{2}{*}{ S1 } & V1 & S1 \\
& V2 & S2 \\
& $\mathrm{V} 3$ & $\mathrm{~S} 3$ \\
\hline \multirow{2}{*}{$\sim$} & $\mathrm{V} 1$ & $\mathrm{~S} 4$ \\
& $\mathrm{~V} 2$ & $\mathrm{~S} 5$ \\
& $\mathrm{~V} 3$ & $\mathrm{~S} 4$ \\
\hline
\end{tabular}

- The Orchestrator: The Orchestrator or the slice manager is responsible for 
managing slices and facilitating on-demand slice allocation, deallocation ${ }^{2}$ and coordinating different components of the framework and user interactions.

- Optimization Module: The optimization module implements our optimization model (section $\S 6.4$ ). It reads the current state of the system allocation that includes remaining $\mathrm{CPU}$, link bandwidth, and delays as well as network topology and processes the incoming request. If a solution is found, the allocation is stored in a database, and the system allocation statistics are updated accordingly.

- Database (DB): The database stores request information, allocation schemes, remaining system resources, and performance statistics. Table 8.1 and 8.2 show an example of CPU requests and allocations database, respectively. Similarly, other tables in the database contain the requested slice bandwidth, allocated bandwidth, and remaining system resources tables.

- O Agent: The Optimization agent (O Agent) is responsible for communicating with the orchestrator and the optimization module. It receives the slice allocation from the orchestrator and forwards it to the optimization module and communicate back the results.

- H Agent: The Hypervisor Agent (H Agent) is an integral part of the framework (runs on each hypervisor). It is responsible for allocating and deallocating slices in real-time, starting applications for each slice, and reporting slice statistics to the orchestrator.

\footnotetext{
${ }^{2}$ DSAF can deallocate slices. However, we have not performed experiments to show the deallocation of slices.
} 


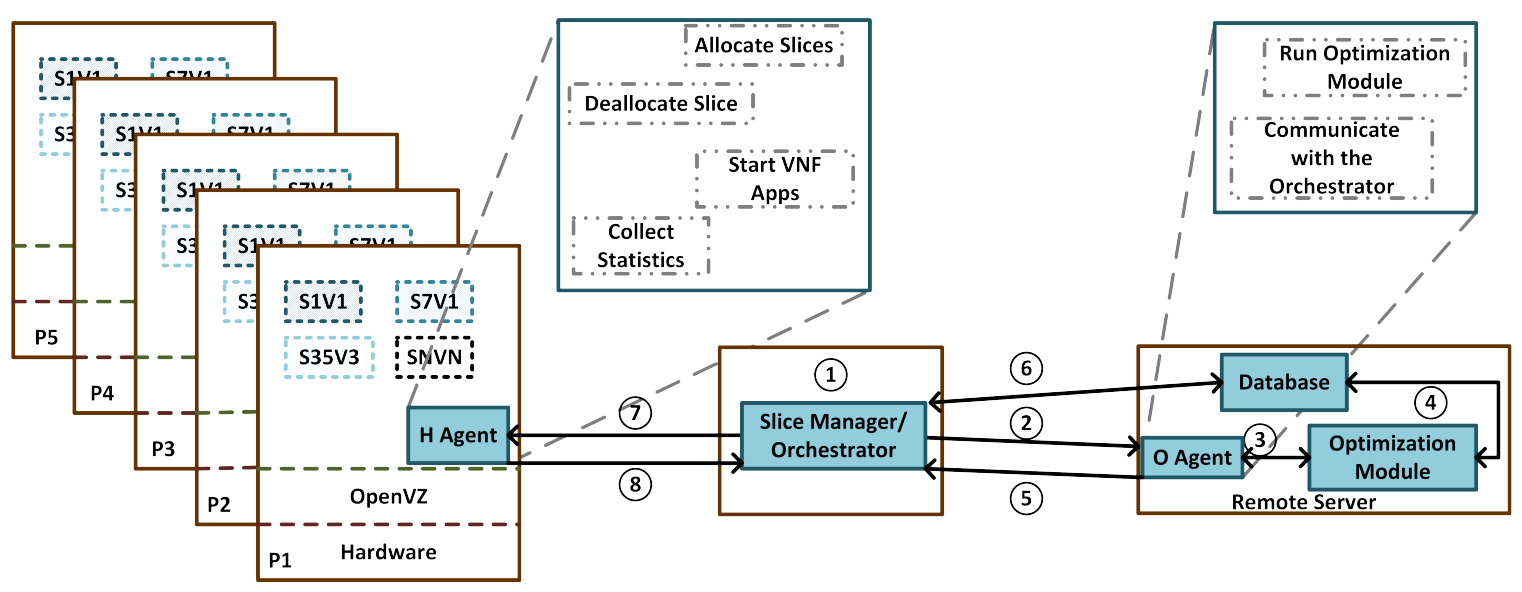

Figure 8.1: DSAF Logical Topology in our experiments: The brown boxes represent the physical servers and blue boxes represent the framework components. Solid lines show the logical communication paths between framework components

The dynamic slice allocation process is shown in Fig. 8.1 and it is described in the following steps (each step corresponds to the circled number in Fig. 8.1):

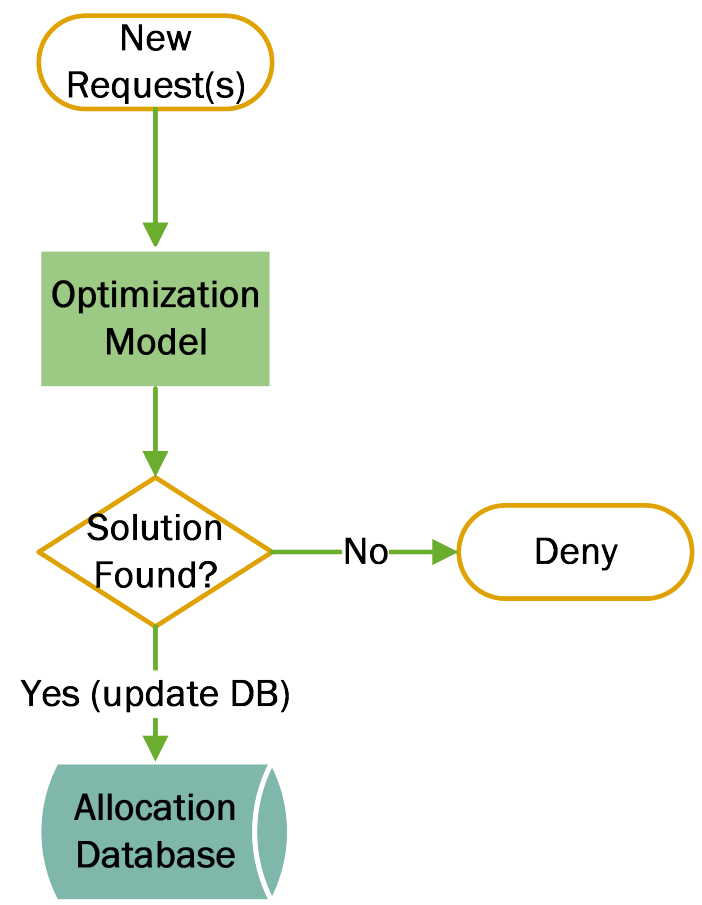

Figure 8.2: Dynamic Slice Allocation Optimization Flow Diagram

1. The orchestrator provides user-interaction and waits for a slice request 
2. Once a slice request is received, it interacts with the O Agent to find the allocation scheme.

3. The $\mathrm{O}$ Agent starts an instance of the optimization model and pass the slice request to the optimization module. The optimization module reads the current network state from the database and finds the best solution to allocate the slice (if feasible). If no solution is found, the request is denied, and the response is sent to the orchestrator. The flow diagram of the allocation process is shown in Fig. 8.2.

4. If a solution is found in step 3, then the slice allocation will be stored in a database.

5. The orchestrator receives an accepted or denied response from the O Agent.

6. If the response is slice request accepted, the orchestrator retrieves the allocation scheme from the database and sorts the retrieved allocation scheme according to the slice(s) that will be allocated on each hypervisor.

7. The orchestrator sends the information to target $\mathrm{H}$ Agent(s). The information includes slice name, IP address, CPU (GHz), HDD, RAM, bandwidth, and any application to start after creating VNF.

8. If the allocation is successful, the $\mathrm{H}$ Agent sends a successful response to the orchestrator

This process is repeated for every request, although DSAF should be able to process multiple requests at the same time. 


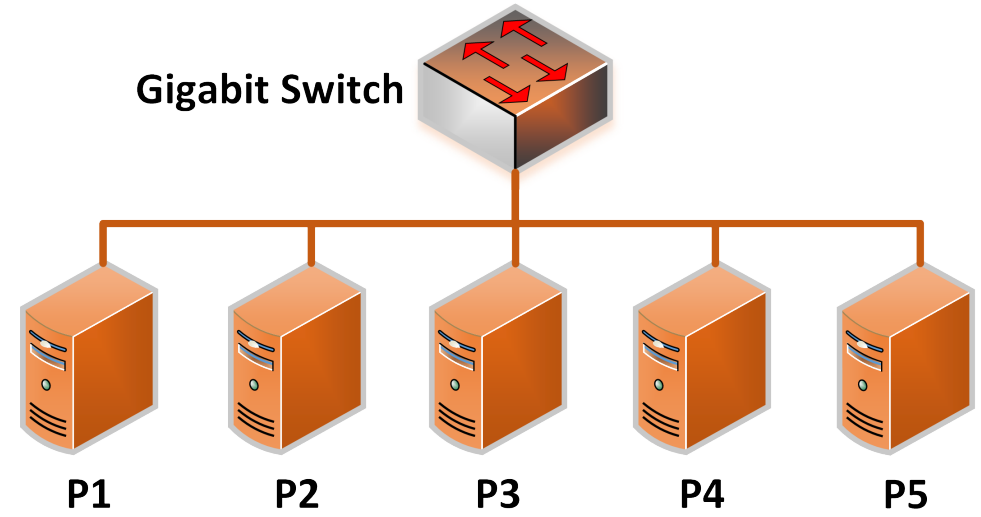

Figure 8.3: DSAF Physical Topology. All links are 1 Gbps

Table 8.3: Experiment Topology Hardware Specification

\begin{tabular}{c|c}
\hline Server(s) & Hardware Specs \\
\hline Remote Server & CPU: 8 cores, RAM: 32GB, Bandwidth: 1Gbps \\
\hline P1 to P3, and the orchestrator & CPU: 4 cores, RAM: 8GB, Bandwidth: 1Gbps \\
\hline P4 and P5 & CPU: 8 cores, RAM: 8GB, Bandwidth: 1Gbps \\
\hline \hline
\end{tabular}

\subsubsection{Experimental Setup}

To evaluate DSAF, we created a testbed using seven servers where five servers (P1 to P5) are used to allocate slices. We are aware that our topology is different from the typical NFV architecture framework, such as Management and Orchestration (MANO) [69], but for simplicity of implementation, we use topology, as shown in Fig. 8.3.

The optimization module and the database are hosted on the same server (Remote Server), and the orchestrator is hosted on a separate server. The hardware specification for all nodes are listed in Table 8.3, and the total resources available for the allocation across all servers are listed in Table 8.4. The slice request parameters are listed in Table 8.5. For simplicity, each request arrives every three seconds (minimum 
Table 8.4: Total Available Resources for Allocation Across All Servers

\begin{tabular}{c|c}
\hline Resource & Capacity \\
\hline CPU Cores & 28 Cores \\
\hline CPU $(\mathrm{GHz})$ & $74.8 \mathrm{GHz}$ \\
\hline RAM & $40 \mathrm{~GB}$ \\
\hline Hard Drive & $1 \mathrm{~TB}$ \\
\hline Total Bandwidth & $5 \mathrm{Gbps}$ \\
\hline \hline
\end{tabular}

time required to allocate NFs on hypervisors), and they are allocated in the order of arrival. We note that slice requests do not expire.

Table 8.5: Slice Request Parameters

\begin{tabular}{r|l}
\hline Parameter & Value \\
\hline Intra-slice isolation & 1,2, or 3 \\
\hline VNF/Slice & 3 \\
\hline Bandwidth & $40-60 \mathrm{Mbps}$ \\
\hline CPU & $0.75-2 \mathrm{GHz}$ \\
\hline Total Slice Requests & 34 \\
\hline \hline
\end{tabular}

We implemented the DSAF in Python [70] and MATLAB [71]. The orchestrator, $\mathrm{O}$, and $\mathrm{H}$ agents are written in Python, and the Optimization module is written in MATLAB. AMPL [51] is used to model the optimization algorithm, and CPLEX 12.9.0 is used as a MILP solver. OpenVZ [52] is used for virtualization. It is an open-source container-based virtualization platform. OpenVZ allows each container to have a specific amount of CPU, RAM, and Hard Drive (HDD). Each container (which hosts one VNF) performs and executes like a stand-alone server. We have 
installed the CentOS 6 [53] operating system in every container. We used Linux Traffic control $(t c)$ [54] to allocate bandwidth for each container.

\subsubsection{Results and Discussion}

Table 8.6: Experiment Scenarios

\begin{tabular}{c|l}
\hline Scenario & Description \\
\hline K1 & one VNF/hypervisor/slice \\
K2 & two or less VNFs/hypervisor/slice \\
K3 & three or less VNFs/hypervisor/slice \\
\hline
\end{tabular}

We used three scenarios to allocate slices, as listed in Table 8.6. In each scenario, we compare the DSAF with the First Come First Serve First Available (FCFSFA). We collected statistics in terms of total slice requests allocated, processing time, and average computation time per slice in all scenarios. In the first scenario, we restricted the allocation to one $\mathrm{VNF} /$ hypervisor per slice $(K 1)$. In the second scenario, only two or fewer VNFs of a slice $(K 2)$ can be allocated on a single hypervisor. The third scenario, three or less VNFs/slice, can be placed on one hypervisor (K3). In FCFSFA, a slice is allocated based on arrival time and the first available server. We make sure that allocated resources do not exceed the available physical resources. However, FCFSFA cannot guarantee the end-to-end delay. We wrote a Python script to perform allocation for FCFSFA. The FCFSFA is implemented on the same server as the orchestrator.

Fig 8.4 shows slice allocation for all scenarios. In scenario K3, all requests are 


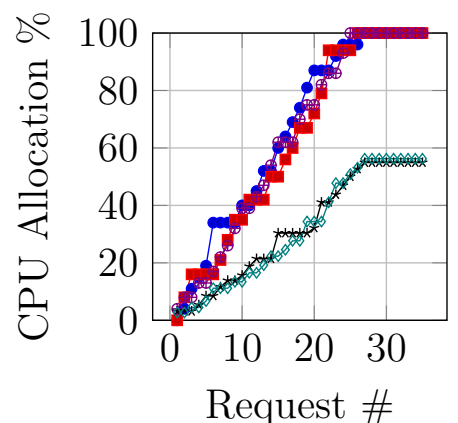

(a) K1 (DSAF)

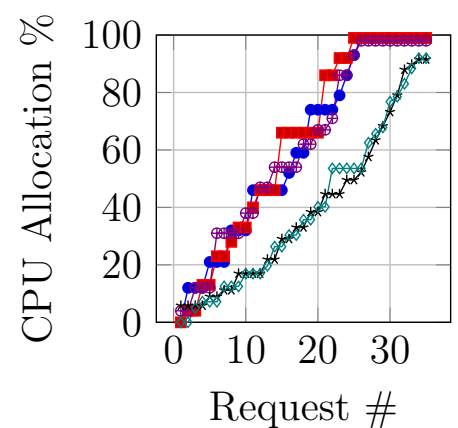

(b) K2 (DSAF)

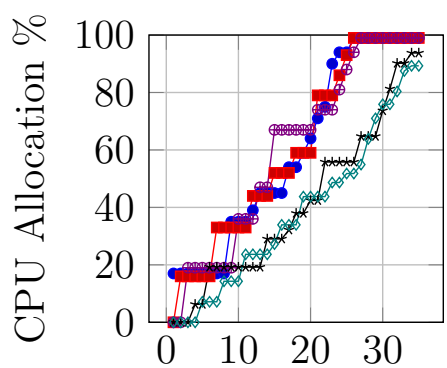

Request \#

(c) K3 (DSAF)

$$
-\mathrm{P} 1 \rightarrow \mathrm{P} 2 \rightarrow \mathrm{P} 3 \rightarrow \mathrm{P} 4 \rightarrow \mathrm{P} 5
$$
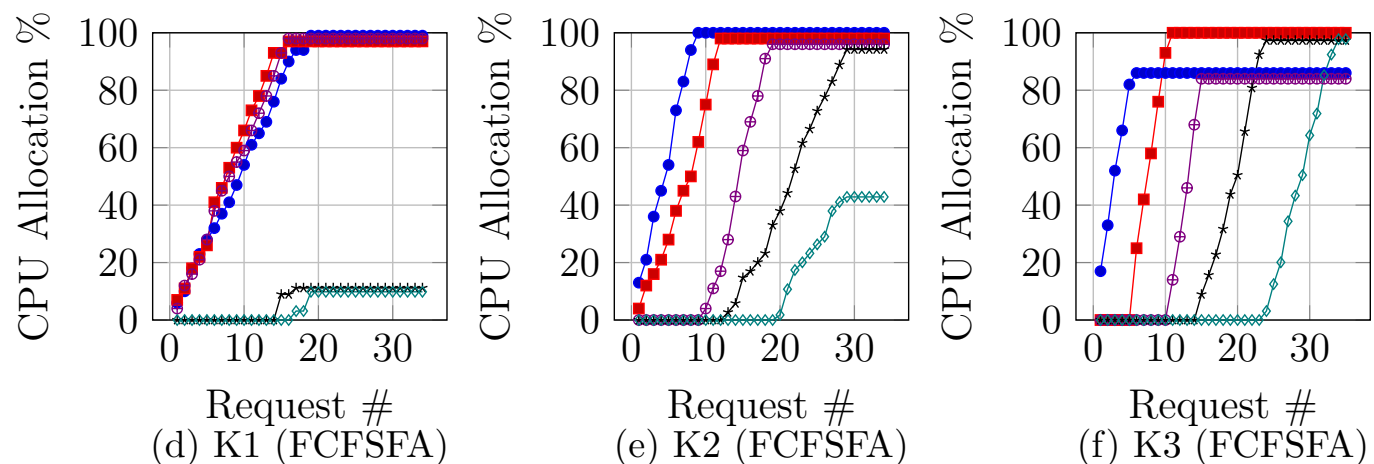

Figure 8.4: Comparison of Allocation Schemes for Different Scenarios

successfully allocated for both allocation schemes, as shown in Fig. 8.4c and 8.4f. However, in scenario K1, DSAF and FCFSFA only allocated 27 and 17 slice requests respectively before P1, P2 and P3 ran out of CPU capacity. For scenario K1, we can only allocate one $\mathrm{VNF}$ /hypervisor per slice; therefore, once these three hypervisors ran out of CPU capacity, we cannot allocate any more slices even though P4 and P5 still have significant resources available (because each slice needs three hypervisors for allocation). An interesting observation to note in Fig. 8.4 is that FCFSFA (all scenarios) allocates slices in an unbalanced manner. This allocation scheme behaves like a greedy approach, where it will allocate slices at the first available hypervisor. It resulted in a lower number of requests being allocated in K1 and K2 as shown in 


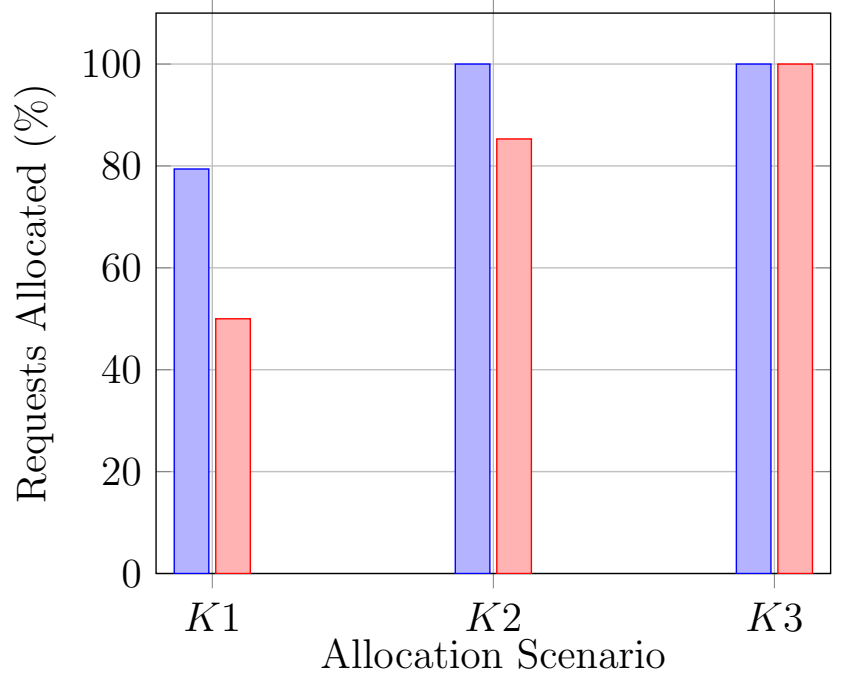

$[$ DSAF $\square$ FCFSFA

Figure 8.5: Total Slice Requests Allocated

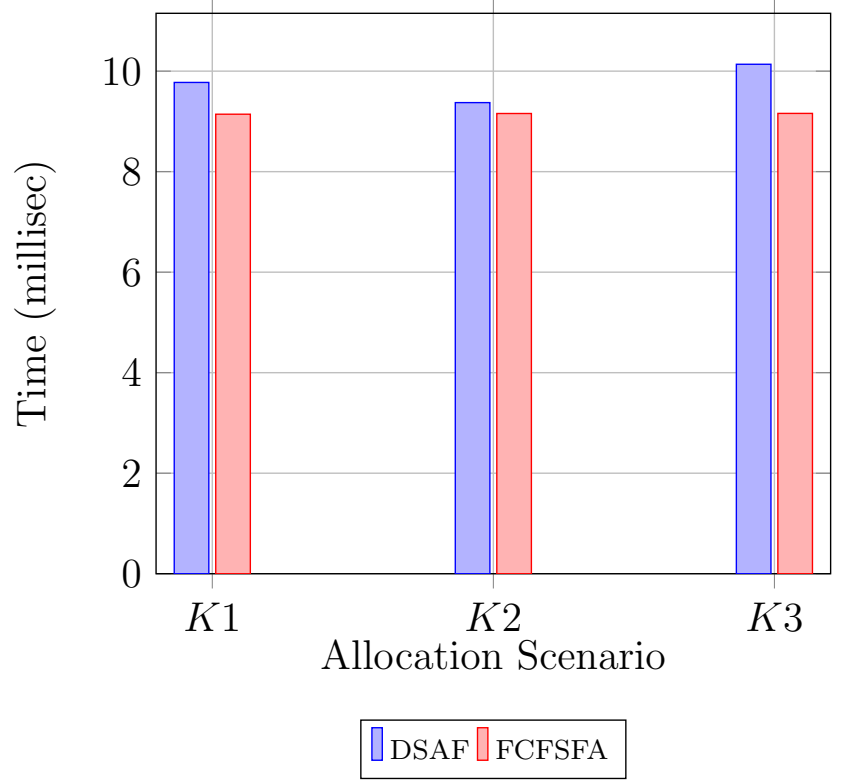

Figure 8.6: Overhead: Processing Time

Fig. $8.4 \mathrm{~d}$ and $8.4 \mathrm{e}$ respectively. It could also result in slices competing for resources on one hypervisor sooner, even though the rest of the system is idle as well as a higher chance of slice unavailability if a hypervisor malfunctions. Whereas DSAF optimally allocates slices in all scenarios and spreads them across the entire system leading to 
less resource contention between slices and in case of a hypervisor malfunction, there is a higher chance that slices could remain partially or fully available.

DSAF can allocate more or equal number of slice requests in all scenarios, as shown in Fig. 8.5.

Fig. 8.6 shows the DSAF and FCFSFA processing time overhead. The processing time includes the time required to process the user requests and send and receive information from the $\mathrm{H}$ and $\mathrm{O}$ agents. For FCFSFA, the processing time is the time required to retrieve allocation requests and read system topology. Although DSAF requires slightly more processing time because of the communication required between the framework's components, it still performs comparably to the FCFSFA in all scenarios.

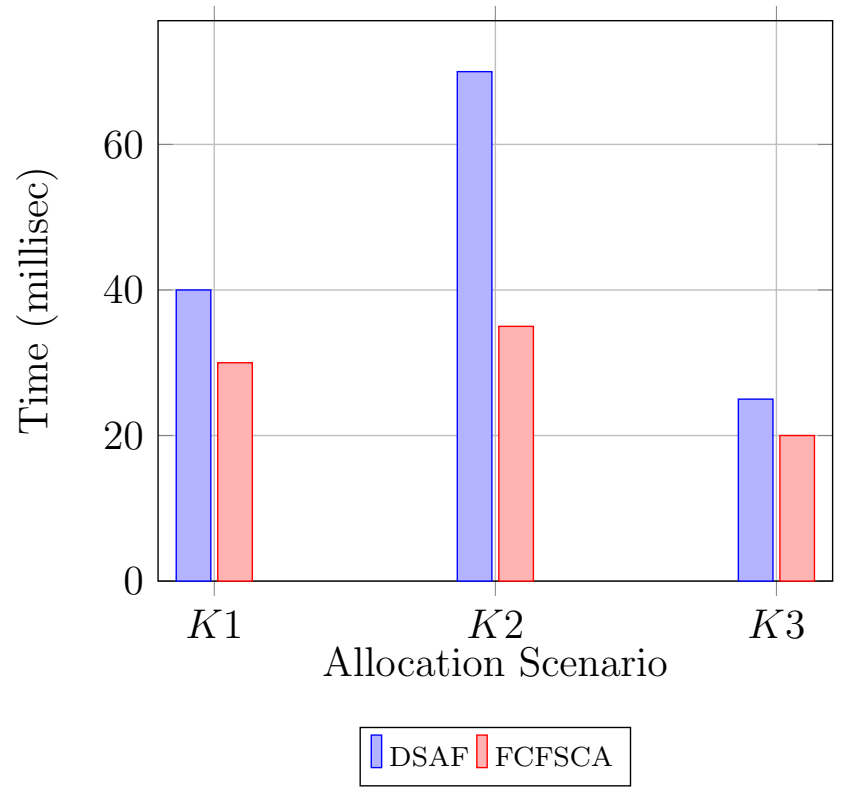

Figure 8.7: Overhead: Average Computation Time Per Slice

The average computation time per slice for DSAF is measured in the optimization module. The average computation time per slice for FCFSFA is the time required to calculate the allocation of slices and updating DB records. FCFSFA has lower 
average computation times per slice because no optimization is performed, as shown in Fig. 8.7. DSAF's average computation time per slice is the cost of allocating more slices as well as providing flexibility when allocating slices.

\subsection{Qualitative Comparison}

In this section, we compare our work with state-of-the-art. We note that the following discussion is based on our understating of the literature but not on any simulation or experiment results.

D. Dietrich et al. [37] proposed a linear programming formulation for the placement of VNFs in the LTE core network ${ }^{3}$. In the proposed optimization model, the $\mathrm{CPU}$ and bandwidth were used as the objective function to perform allocation and latency between the network functions. Whereas our proposed optimization model used the CPU and latency as objective function while providing slice isolation and guaranteeing the end-to-end delay for a $5 \mathrm{G}$ mobile core slice. We believe that $\mathrm{D}$. Dietrich et al.'s proposed optimization model might be more efficient in average computation time because the optimization model has fewer constraints, and the model is relaxed from MILP to LP, which could reduce computation time. However, the number of requests accepted should be similar to our allocation model if we do not use inter-slice isolation and intra-slice isolation is relaxed (i.e., $\left.K_{r e l}=10\right)$ for the same dataset. Another important parameter is the latency. Our proposed optimization model should provide better allocation since we consider link delay, VNF, and hypervisor processing delay. Whereas D. Dietrich et al. only considered link delays.

\footnotetext{
${ }^{3}$ We are comparing our work with D. Dietrich et al.'s work because the proposed optimization solution in Chap. 5 extends their work for $5 \mathrm{G}$ mobile network and use some of the constraints in Chap. 6
} 
The Joint Edge and Central Resource Slicer (JECRS) was proposed for end-toend slicing as service (i.e., RAN, transport network, and central office) in [72]. The proposed framework considered communication and computing resources of the Multiaccess Edge Computing (MEC) architecture. The slice resources were translated from high-level requirements to specific resources for automated allocation in MEC architecture. Our proposed allocation framework share some similarities with the JECRS. Both frameworks provide user-interaction to obtain slice requirements and translate into specific resource requirements for dynamic allocation. One of JECRS framework's main advantages is that it provides slicing for RAN, transport network, and core network, whereas DSAF only considers the core network slice. However, the actual resource allocation scheme is not provided, and although latency is considered in the proposed JECRS framework, the authors did not provide any detail on how it is guaranteed. A similar case with the isolation, where the effect of isolation is discussed, but no details are provided on how isolation is achieved. On the other hand, in DSAF, we guarantee the end-to-end delay and provide a detailed discussion on the different types of slice isolation (i.e., intra-slice and inter-slice $)^{4}$. The JECRS framework has an advantage because it can allocate slice end-to-end (i.e., RAN, transport, and core), whereas DSAF can only allocate core slices. However, DSAF can optimally allocate slices and guarantee resources and performance parameters at the time of allocation, which are features absent from the JECRS framework.

\footnotetext{
${ }^{4}$ Detailed discussion in Chap. 6
} 


\subsection{Summary}

In this chapter, we presented a dynamic slice allocation framework to allocate slices in a resource-efficient manner. The framework provides automation for slice allocation. We compared our framework with the First Come First Serve First Available allocation scheme. The evaluation of both techniques was done on a real testbed. Our results show that the overall proposed framework has comparable overhead to the FCFSFA. The cost of running DSAF is the average computation time that is slightly higher then FCFSFA. However, DSAF can allocate significantly more slices as well as it can fulfill a few requirements of the 5G (e.g., end-to-end delay). DSAF allocates slices in a balanced manner across the network, which means less resource contention between slices until the network reaches a saturation state. In FCFSFA, the resource contention could happen prematurely because slices are allocated in an unbalanced fashion (i.e., more slices on one hypervisor then the other). 


\section{Chapter 9}

\section{Conclusion}

66At the end of the day, the goals are simple: safety and security. 99

Jodi Rell

Efficient resource management in virtualized networks is essential for a costeffective solution. There are many advantages and disadvantages to virtualized networks. Security is one of the weaknesses of virtual networks, but it is essential and being improved every day. However, attacks on the networks are also getting frequent and sophisticated. DDoS attacks are easier to perform and harder to defend. So there is always a need to keep innovating new solutions to defend against DDoS attacks.

In this dissertation, we focused on the security of the virtualized networks. In particular, we explore the impact of DDoS attacks on service availability in SDN and 5G mobile networks and co-residency issue in 5G networks. We began by performing experiments in SDN to observe the service disruption due to DDoS attacks. After observing, we proposed a solution that took advantage of SDN programmability and flexibility to mitigate DDoS attacks. Our proposed solution, Adaptive Bubble Burst (ABB), used load-balancing to mitigate DDoS attacks in SDN. Our experimental results showed that using adaptive resource management resulted in better service 
availability under DDoS attack and improved end-user experience by enhancing response time.

We realized that efficient resource management could be used to mitigate DDoS attacks in virtualized networks. The next-generation mobile network (5G) relies on a virtualized core network to provide various services by employing networking slicing. We proposed an optimization-based resource allocation solution to mitigate DDoS attacks as well as to provide efficient resource allocation. Our solution used two types of slice isolation (i.e., intra-slice and inter-slice) to provide flexible DDoS mitigation and slice allocation. We used simulations to measure the proposed solution's performance and a testbed to see the effectiveness of our solution in mitigating DDoS attacks. The bandwidth and resource exhaustion DDoS attacks were used to evaluate our solution. Our results showed that slice isolation could be used to mitigate both types of attacks effectively.

After performing various types of experiments for DDoS attacks and observing the effectiveness of our proposed solution, we further explored the security issue of malicious co-residency that could be used to launch DDoS attacks or side-channel attacks. We analyzed the defense against malicious co-residency using our optimization-based resource allocation. Our simulation results showed that our proposed optimizationbased resource allocation scheme provides proactive defense against undesired malicious co-residency with no additional computational cost, especially at higher system resource utilization.

Various components of the proposed solution were performed manually, which reduced efficiency in running and implementing the solution. We developed a framework (DSAF) for allocating slices in an automated and dynamic fashion to resolve this issue. We compared our framework with the First Come First Server First Available 
in terms of allocation efficiency and performance. The results showed that our proposed framework was significantly more efficient in allocating slices, but it required a slightly higher computation time.

\subsection{Current Limitations}

As it is for every research, this dissertation has the following limitations:

- In the SDN network, our proposed solution (ABB) only relied on incoming traffic rate to determine if a service is under DDoS attack. The incoming traffic rate parameter could lead to false positives because, in a situation when there is a flash crowd, the traffic rate would be much higher than normal traffic. Another limitation of ABB is that when all available resources allocated to service are at maximum utilization, traffic is dropped without a distinction between legitimate traffic and DDoS traffic, leading to intermittent service unavailability to endusers.

- Our proposed slice allocation solution only guarantees allocated resources (SLA) in the network's current state. For instance, the latency in the network could keep changing with the increased traffic load that would impact the end-to-end delay for a slice. Further traffic engineering is required to guarantee SLAs.

- In the 5G network, our resource allocation based solution provided proactive against DDoS attacks and co-residency. However, reactive solutions would be needed (e.g., migrating slices under DDoS attacks). In such cases, DDoS detection will play a crucial role. For instance, if a slice is under DDoS attack and slice migration is used as a mitigation solution, then accurate DDoS detection will be significant because a false identification of a slice under DDoS attack 
could lead to an undesirable outcome. The DDoS detection could also play an essential role in reactive mitigation for malicious co-residency, as discussed in section $\S 7.5$. In which we need to profile the legitimate behavior of the users and identify anomalies in the user requests to thwart malicious co-residency attempts. Therefore, DDoS detection and its impact on our proposed solution require further study.

- Slice allocation model might require further optimization to be deployed on a large scale to improve performance. 


\section{Bibliography}

[1] J. Sahoo, S. Mohapatra, and R. Lath, "Virtualization: A survey on concepts, taxonomy and associated security issues," in 2010 Second International Conference on Computer and Network Technology, pp. 222-226, April 2010.

[2] J. G. Herrera and J. F. Botero, "Resource allocation in nfv: A comprehensive survey," IEEE Transactions on Network and Service Management, vol. 13, no. 3, pp. 518-532, 2016.

[3] X. Li, M. Samaka, H. A. Chan, D. Bhamare, L. Gupta, C. Guo, and R. Jain, "Network slicing for 5g: Challenges and opportunities," IEEE Internet Computing, vol. 21, no. 5, pp. 20-27, 2017.

[4] B. S. Davie, L. L. Peterson, and D. Clark, Computer Networks: A Systems Approach. Morgan Kaufmann, 2007.

[5] W. Xia, Y. Wen, C. H. Foh, D. Niyato, and H. Xie, "A survey on softwaredefined networking," IEEE Communications Surveys Tutorials, vol. 17, pp. 2751, Firstquarter 2015.

[6] J. De Vriendt, P. Lainé, C. Lerouge, and X. Xu, "Mobile network evolution: a revolution on the move," IEEE Communications magazine, vol. 40, no. 4, pp. 104-111, 2002 . 
[7] C. Cox, An introduction to LTE: LTE, LTE-advanced, SAE and $4 G$ mobile communications. John Wiley \& Sons, 2012.

[8] M. Bennis et al., "When cellular meets WiFi in wireless small cell networks," IEEE Comm. Magazine, vol. 51, pp. 44-50, June 2013.

[9] R. Borgaonkar et al., "Femtocells: A Poisonous Needle in the Operator's Hay Stack." presented at BlackHat, Las Vegas, July 2011.

[10] A. Golaup et al., "Femtocell access control strategy in UMTS and LTE," IEEE Comm. Magazine, vol. 47, pp. 117-123, September 2009.

[11] D. Sattar, A. Matrawy, T. Bryant, and M. Kneppers, "Threat Modeling in LTE Small Cell Networks," in 2018 IEEE Canadian Conference on Electrical \&3 Computer Engineering (CCECE), pp. 1-5, IEEE, 2018.

[12] P. Popovski and V. Brau and H.-P. Mayer and P. Fertl and Z. Ren and D. Gonzales-Serrano and E. G. Strom and T. Svensson and H. Taoka and P. Agyapong, "EU FP7 INFSO-ICT-317669 METIS, D1.1: Scenarios, requirements and KPIs for 5G mobile and wireless system," TS 1, Mobile and wireless communications Enablers for the Twenty-twenty Information Society, April 2013.

[13] A. Gupta and R. K. Jha, "A survey of 5g network: Architecture and emerging technologies," IEEE Access, vol. 3, pp. 1206-1232, 2015.

[14] Marco Carugi, "Key features and requirements of 5G/IMT2020 networks." $\quad$ https://www.itu.int/en/ITU-D/RegionalPresence/ArabStates/Documents/events/2018/RDF/WorkshopFebruary 2018.

[15] 5G Americas, "5G Americas White Paper Network Slicing for 5G and Beyond," TS, 5G Americas, November 2016. 
[16] 3GPP, "System Architecture for the 5G System;Stage 2," TS 23.501 V15.4.0, 3GPP, Dec 2018.

[17] Zoom, "Zoom." https://zoom.us/. Accessed July 2020.

[18] Microsoft, "Teams." https://teams.microsoft.com. Accessed July 2020.

[19] Goole, "Meet." https://meet.google.com/. Accessed July 2020.

[20] P. Zaroo, "A survey of ddos attacks and some ddos defense mechanisms," 2002.

[21] S. Scott-Hayward, S. Natarajan, and S. Sezer, "A Survey of Security in Software Defined Networks," Communications Surveys Tutorials, IEEE, vol. PP, no. 99, pp. 1-1, 2015.

[22] Z. Hu, M. Wang, X. Yan, Y. Yin, and Z. Luo, "A comprehensive security architecture for SDN," in Intelligence in Next Generation Networks (ICIN), 2015 18th International Conference on, pp. 30-37, Feb 2015.

[23] C. Douligeris and A. Mitrokotsa, "DDoS attacks and defense mechanisms: classification and state-of-the-art," Computer Networks, vol. 44, no. 5, pp. 643 - 666, 2004

[24] S. T. Zargar, J. Joshi, and D. Tipper, "A survey of defense mechanisms against distributed denial of service (ddos) flooding attacks," IEEE Communications Surveys Tutorials, vol. 15, pp. 2046-2069, Fourth 2013.

[25] J. H. Jafarian, E. Al-Shaer, and Q. Duan, "Openflow Random Host Mutation: Transparent moving target defense using software defined networking," in Proceedings of the First Workshop on Hot Topics in Software Defined Networks, HotSDN'12, (New York, NY, USA), pp. 127-132, ACM, 2012. 
[26] N.-N. Dao, J. Park, M. Park, and S. Cho, "A feasible method to combat against DDoS attack in SDN network," in Information Networking (ICOIN), 2015 International Conference on, pp. 309-311, Jan 2015.

[27] S. Lim, J. Ha, H. Kim, Y. Kim, and S. Yang, "A SDN-oriented DDoS blocking scheme for botnet-based attacks," in Ubiquitous and Future Networks (ICUFN), 2014 Sixth International Conf on, pp. 63-68, July 2014.

[28] V. Krylov, K. Kravtsov, E. Sokolova, and D. Lyakhmanov, "SDI defense against DDoS attacks based on ip fast hopping method," in Science and Technology Conference (Modern Networking Technologies) (MoNeTeC), 2014 International, pp. 1-5, Oct 2014 .

[29] A. Passito, E. Mota, R. Bennesby, and P. Fonseca, "Agnos: A framework for autonomous control of software-defined networks," in 2014 IEEE 28th International Conference on Advanced Information Networking and Applications, pp. 405-412, May 2014.

[30] D. Kewley, R. Fink, J. Lowry, and M. Dean, "Dynamic approaches to thwart adversary intelligence gathering," in DARPA Information Survivability Conference amp; Exposition II, 2001. DISCEX '01. Proceedings, vol. 1, pp. 176-185 vol.1, 2001.

[31] S. Antonatos, P. Akritidis, E. P. Markatos, and K. G. Anagnostakis, "Defending against hitlist worms using network address space randomization," in Proceedings of the 2005 ACM Workshop on Rapid Malcode, WORM '05, (New York, NY, USA), pp. 30-40, ACM, 2005.

[32] Stanford, "POX wiki." https://openflow.stanford.edu/display/ONL/POX+Wiki. Accessed December 2015. 
[33] J. Kneschke, "Lighty." https://www.lighttpd.net/. Accessed March 2016.

[34] X. de Foy and A. Rahman, "Network Slicing - 3GPP Use Case," RFC, RFC Editor, October 2017.

[35] Q. Li, G. Wu, A. Papathanassiou, and M. Udayan, "An end-to-end network slicing framework for 5G wireless communication systems," CoRR, vol. abs/1608.00572, 2016.

[36] Z. Kotulski et al., "On end-to-end approach for slice isolation in 5G networks. Fundamental challenges," in Federated Conf. on Computer Science and Information Systems (FedCSIS), 2017.

[37] D. Dietrich et al., "Network function placement on virtualized cellular cores," in 9th International Conf. on Comm. Sys. and Networks, 2017.

[38] L. Zanzi and V. Sciancalepore, "On Guaranteeing End-to-End Network Slice Latency Constraints in 5G Networks," in 15th International Symposium on Wireless Communication Systems (ISWCS), 2018.

[39] V. N. Sathi et al., "A Novel Protocol for Securing Network Slice Component Association and Slice Isolation in 5G Networks," in Proceedings of the 21st ACM International Conference on Modeling, Analysis and Simulation of Wireless and Mobile Systems, 2018.

[40] Y. Khettab and others, "Virtual security as a service for 5g verticals," in IEEE Wireless Comm. and Networking Conf., 2018.

[41] J. Ni et al., "Efficient and Secure Service-Oriented Authentication Supporting Network Slicing for 5G-Enabled IoT," IEEE Journal on Selected Areas in Communications, vol. 36, no. 3, 2018. 
[42] Z. Kotulski et al., "Graph-Based Quantitative Description of Networks' Slices Isolation," in Federated Conference on Computer Science and Information Systems (FedCSIS), 2018.

[43] V. Sciancalepore et al., "ONETS: Online Network Slice Broker From Theory to Practice," ArXiv e-prints, 2018.

[44] R. Ford, A. Sridharan, R. Margolies, R. Jana, and S. Rangan, "Provisioning low latency, resilient mobile edge clouds for 5G," in 2017 IEEE Conference on Computer Communications Workshops (INFOCOM WKSHPS), pp. 169-174, May 2017.

[45] G. Garcia-Aviles, M. Gramaglia, P. Serrano, and A. Banchs, "Posens: A practical open source solution for end-to-end network slicing," IEEE Wireless Communications, vol. 25, pp. 30-37, October 2018.

[46] Q. Yan et al., "Software-Defined Networking (SDN) and Distributed Denial of Service (DDoS) Attacks in Cloud Computing Environments: A Survey, Some Research Issues, and Challenges," IEEE Comm. Surveys Tutorials, vol. 18, no. 1, 2016

[47] S. Basu, A. Bardhan, K. Gupta, P. Saha, M. Pal, M. Bose, K. Basu, S. Chaudhury, and P. Sarkar, "Cloud computing security challenges \& solutions-A survey," in IEEE 8th Annual Comp. and Comm. Workshop and Conf. (CCWC), 2018.

[48] "Feasibility Study on New Services and Markets Technology Enablers; Stage 1 ," TS 33.8891 v1.3.0, 3GPP, August 2017.

[49] "Study on the security aspects of the next generation system (Release 14)," TS 33.8891 v14.2.0, 3GPP, September 2016. 
[50] D. Sattar and A. Matrawy, "Optimal slice allocation in 5 g core networks," IEEE Networking Letters, vol. 1, pp. 48-51, June 2019.

[51] "A Modeling Language for Mathematical Programming." Accessed January 2019.

[52] “OpenVZ." https://openvz.org/. Accessed January 2019.

[53] "CentOS." https://www.centos.org/. Accessed January 2019.

[54] B. Hubert, "Traffic Control (tc)." https://linux.die.net/man/8/tc. Accessed January 2019.

[55] "ns3." https://www.nsnam.org/. Accessed January 2019.

[56] A. Turner, "tcpreplay." https://linux.die.net/man/1/tcpreplay. Accessed January 2019 .

[57] M. Mezzavilla et al., "End-to-End Simulation of 5G mmWave Networks," IEEE Communications Surveys Tutorials, vol. 20, no. 3, 2018.

[58] J. Dugan et al., "iperf." https://iperf.fr/. Accessed January 2019.

[59] D. Sattar and A. Matrawy, "Towards Secure Slicing: Using Slice Isolation to Mitigate DDoS Attacks on 5G Core Network Slices," in 7th Annual IEEE Conference on Communications and Network Security (CNS 2019), June 2019.

[60] NGMN Alliance, "An Introduction to Network Slicing." https://www.ngmn.org/fileadmin/ngmn/content/downloads/Technical/2015 /NGMN_5G_White_Paper_V1_0.pdf. Accessed November 2017.

[61] P. Rost, C. Mannweiler, D. S. Michalopoulos, C. Sartori, V. Sciancalepore, N. Sastry, O. Holland, S. Tayade, B. Han, D. Bega, D. Aziz, and H. Bakker, 
"Network slicing to enable scalability and flexibility in 5g mobile networks," IEEE Communications Magazine, vol. 55, pp. 72-79, May 2017.

[62] S. Zhang, "An Overview of Network Slicing for 5G," IEEE Wireless Communications, vol. 26, pp. 111-117, June 2019.

[63] A. O. F. Atya, Z. Qian, S. V. Krishnamurthy, T. L. Porta, P. McDaniel, and L. Marvel, "Malicious co-residency on the cloud: Attacks and defense," in IEEE INFOCOM 2017 - IEEE Conference on Computer Communications, pp. 1-9, May 2017.

[64] Z. Wu, Z. Xu, and H. Wang, "Whispers in the hyper-space: High-speed covert channel attacks in the cloud," in Presented as part of the 21st USENIX Security Symposium (USENIX Security 12), pp. 159-173, 2012.

[65] D. Gruss, R. Spreitzer, and S. Mangard, "Cache template attacks: Automating attacks on inclusive last-level caches," in 24th USENIX Security Symposium (USENIX Security 15), (Washington, D.C.), pp. 897-912, USENIX Association, Aug. 2015.

[66] A. Bates, B. Mood, J. Pletcher, H. Pruse, M. Valafar, and K. Butler, "Detecting co-residency with active traffic analysis techniques," in Proceedings of the 2012 ACM Workshop on Cloud Computing Security Workshop, CCSW 12, (New York, NY, USA), p. 112, Association for Computing Machinery, 2012.

[67] A. O. F. Atya, Z. Qian, S. V. Krishnamurthy, T. La Porta, P. McDaniel, and L. M. Marvel, "Catch me if you can: A closer look at malicious co-residency on the cloud," IEEE/ACM Transactions on Networking, vol. 27, pp. 560-576, April 2019. 
[68] Y. Zhang, A. Juels, A. Oprea, and M. K. Reiter, "Homealone: Co-residency detection in the cloud via side-channel analysis," in 2011 IEEE Symposium on Security and Privacy, pp. 313-328, May 2011.

[69] M. Ersue, "ETSI NFV Management and Orchestration." https://www.ietf.org/proceedings/88/slides/slides-88-opsawg-6.pdf. October 2013 .

[70] Python Software Foundation, "Python." https://www.python.org/. Accessed April 2019.

[71] MathWorks, "MATLAB." https://www.mathworks.com/products/matlab.html. Accessed April 2019.

[72] H. Chien, Y. Lin, C. Lai, and C. Wang, "End-to-end slicing as a service with computing and communication resource allocation for multi-tenant $5 \mathrm{~g}$ systems," IEEE Wireless Communications, vol. 26, no. 5, pp. 104-112, 2019. 